Submitted to The Astrophysical Journal

\title{
A Comparison of the Afterglows of Short- and Long-Duration Gamma-Ray Bursts
}

\author{
M. Nysewander ${ }^{1,2}$, A.S. Fruchter ${ }^{1} \&$ A. Pe'er ${ }^{1,3}$
}

\begin{abstract}
We present a comparative study of the observed properties of the optical and $\mathrm{X}$-ray afterglows of short- and long-duration $\gamma$-ray bursts (GRBs). Using a large sample of 37 short and 421 long GRBs, we find a strong correlation between the afterglow brightness measured after 11 hours and the observed fluence of the prompt emission. Both the optical (R band) and X-ray flux densites $\left(F_{R}\right.$ and $F_{X}$ ) scale with the $\gamma$-ray fluence, $F_{\gamma}$. For bursts with a known redshift, a tight correlation exists between the afterglow flux densities at 11 hours (rest-frame) and the total isotropic $\gamma$-ray energy, $\mathrm{E}_{\gamma, \mathrm{ISO}}: F_{R, X} \propto \mathrm{E}_{\gamma, \mathrm{ISO}}{ }^{\alpha}$, with $\alpha \simeq 1$. The constant of proportionality is nearly identical for long and short bursts, when $\mathrm{E}_{\gamma, \mathrm{ISO}}$ is obtained from the Swift data. Additionally, we find that for short busts with $F_{\gamma} \gtrsim 10^{-7} \mathrm{erg}^{-2}$, optical afterglows are nearly always detected by reasonably deep early observations. Finally, we show that the ratio $F_{R} / F_{X}$ has very similar values for short and long bursts. These results are difficult to explain in the framework of the standard scenario, since they require that either (1) the number density of the surrounding medium of short bursts is typically comparable to, or even larger than the number density of long bursts; (2) short bursts explode into a density profile, $n(r) \propto r^{-2}$ or (3) the prompt $\gamma$-ray fluence depends on the density of the external medium. We therefore find it likely that either basic assumptions on the properties of the circumburst environment of short GRBs or else the standard models of GRB emission must be re-examined. We believe that the most likely solution is that the ambient density surrounding typical short bursts is higher than has generally been expected: a typical value of $\sim 1$ per $\mathrm{cm}^{-3}$ is indicated. We discuss recent modifications to the standard binary merger model for short bursts which may be able to explain the implied density.
\end{abstract}

\footnotetext{
${ }^{1}$ Space Telescope Science Institute, 3700 San Martin Dr, Baltimore, MD, 21218

${ }^{2}$ Alion Science \& Technology, 1000 Park Forty Plaza, Durham, NC 27713; mnysewander@alionscience.com

${ }^{3}$ Giacconi Fellow
} 
Subject headings: gamma rays: bursts — radiation mechanism: nonthermal

\section{Introduction}

Although the past decade has seen great progress in characterizing the afterglows of longduration GRBs (LGRBs), the afterglows of short-duration GRBs (SGRBs) remain elusive. The average fluence of an SGRB is an order of magnitude fainter than that of an LGRB, and thus while SGRBs have been localized by Swift, about ten long bursts are localized for every short burst. Optical afterglows of SGRBs have been detected, but are notoriously dim, and large-aperture telescopes are generally required to detect them. Nearly half of all SGRBs have a fluence less than $10^{-7} \mathrm{ergs}^{-2}$ and none of these have a detected optical afterglow. SGRB X-ray afterglows are commonly detected, but are faint and less likely to be rapidly localized with their positions distributed in real-time, further complicating optical ground-based observations. As a result, only a few well-sampled, robust optical SGRB light curves exist that span a wide range of time or frequency.

The faintness of the afterglows of short GRBs (compared to the long GRBs) has been attributed to properties of the progenitor and circumburst environment. While the LGRBSN connection is strong, the progenitors of SGRBs are less certain. Observations have shown that long-duration GRBs are associated with the deaths of massive stars (Stanek et al. 2003; Hiorth et al. 2003a; see Woosley \& Bloom 2006 for a comprehensive review). However, a number of models exist for the progenitors of SGRBs. The leading model is a binary pair of compact objects, dual neutron stars, or a neutron star and black hole, which merge in a dramatic explosion causing a burst of $\gamma$-rays (Eichler et al. 1989; Narayan et al. 1992; Lee et al. 2005) powered by accretion onto the newly formed compact object.

It is commonly believed that the difference in the long and short progenitors implies that they reside in different environments (for a recent review, see Nakar 2007). Because LGRBs are associated with massive stars, they will lie in or near dense regions of active starformation. SGRBs progenitors can have long lifetimes and do not need to be in galaxies with active star-formation, nor be associated with dense regions. In fact, the observed diverse host galaxy morphology is roughly consistent with the expected wide range of progenitor lifetimes (Belczynski et al. 2006a).

Two of the first detected SGRB afterglows, those of GRB 050509B (Gehrels et al. 2005) and GRB 050724 (Berger et al. 2005), were found near bright, giant elliptical galaxies. GRB 050709 (Hjorth et al. 2005) and GRB 051221A (Soderberg et al. 2006a) both occurred in late-type galaxies with ongoing star-formation. However, within these late-type galaxies, 
SGRBs do not necessarily trace areas of active star-formation as is the case with LGRBs (Bloom et al. 2002; Fruchter et al. 2006), rather, they can occur in low luminosity outer regions of the galaxy (e.g. GRB 050709; Fox et al. 2005, GRB 060121; Levan et al. 2006b). Some theories of the formation of compact object binaries include a violent supernovainduced kick that can offset them from their host galaxy (Belczynski et al. 2006b) and may place them in these low density environments.

The low environmental densities into which SGRBs occur have an observational implication. Within the context of the standard afterglow model (e.g. Mészáros \& Rees 1997; Sari et al. 1998) for observing frequencies below the cooling frequency (and above the peak frequency), the afterglow flux is proportional to the density of the external medium to the power 1/2. Well-sampled, multiwavelength observations of LGRB afterglows have suggested that at half a day, the cooling frequency generally lies between the optical and Xray frequencies (e.g. Galama et al. 1998b; Harrison et al. 1999; Panaitescu \& Kumar 2002; Soderberg et al. 2006a). It is therefore expected that the differences in the density of the circumburst medium between the long and short bursts would affect the late time optical emission, which is below the cooling break. (Note that according to the standard model, the flux above the cooling break is independent of the external medium density, $n$. Thus, the flux at the X-ray frequency may not be affected by the difference in densities. See discussion in 94 below).

In addition to depending on the environment, we also expect the absolute afterglow brightnesses to be proportional to the total energy released in the burst. The intrinsic energy release of short burst progenitors is not well-known because the current redshift distribution of SGRBs is fragmentary. The average redshift of the SGRB distribution is $\langle z\rangle=0.5$ with $\mathrm{E}_{I S O} \sim 10^{48}-10^{51}$, compared to $\left\langle z>=1.9\right.$ and $\mathrm{E}_{I S O} \sim 10^{49}-10^{53.5}$ for LGRBs. However, it is unclear to what extend these differences are a result of observational biases and/or true intrinsic differences in luminosity or collimation in comparison with LGRBs. No SGRB absorption redshifts have been measured, which is likely due to the lower afterglow brightness and possibly a less dense external medium on which to imprint strong absorption lines. Therefore, because all SGRB redshifts have been measured from optical emission lines in host galaxies, the current sample of redshifts is limited to $z \lesssim 1$. Berger et al. (2007a) have noted that the lack of bright galaxies in the error circles of a number of XRT localizations may suggest that a number of short bursts without optical identifications may lie at $z>1$. Finally we note that the $\mathrm{E}_{I S O}$ values presented here are either based upon the $\gamma$-ray fluence observed by the Swift Burst Alert Telescope (BAT) or for the smaller non-Swift sample, scaled to the BAT sensitivity. Because the BAT is only sensitive up to $150 \mathrm{keV}$, these values underestimate the total prompt emission of all bursts. However, this is most severe on short bursts, which tend to have a harder spectrum than long bursts. We return to this point in 
more detail later.

In this paper we compare the optical and X-ray afterglows measured after 11 hours (a standard Swift timescale) with the prompt energy emission of both long- and short-duration $\gamma$-ray bursts, and compare these results with afterglow theory. We test the predictions of the standard model with a large data set that spans over six decades in energy. We find that SGRBs afterglows are very similar to low-luminosity LGRBs afterglows. Surprisingly, we find that the afterglow brightnesses depend little upon the classification of long or short; rather, the bursts scale primarily with the high-energy prompt emission. Our result is difficult to reconcile with standard afterglow theory, and contradicts previous claims that SGRB optical afterglows are dim due to the density of their surrounding medium (Panaitescu et al. 2001; Nakar 2007; Berger 2007a).

This paper is organized as follows: In $₫ 2$ we detail the steps taken to gather the data presented herein. We compare the prompt and afterglow emission of the two populations in $\S 3$. In $\S 4$, we explore the assumptions of the standard model, under what conditions the results are valid, and implications related to host galaxies and intrinsic SGRB energies. We show that the afterglow data implies that either (1) the environment of short GRBs is comparable to that of long GRBs, (2) SGRBs explode into a wind profile, or (3) the prompt emission fluence depends on the environment. In \$5 we discuss the statistical significance of our data sample. We summarize and draw final conclusions in $₫ 6$.

\section{Data}

In this study, we include GRBs detected by all satellites, beginning with GRB 970111, the first localization with follow-up observations capable of detecting an afterglow, and ending over a decade later, with GRB 071227. To be included in the analysis, a GRB must have published values of the prompt emission and had optical or X-ray afterglow follow-up observations within a few days of the burst. 421 LGRBs and 37 SGRBs have been included in the sample, out of which 408 LGRB and 37 SGRB have optical and 299 LGRBs and 27 SGRB have X-ray follow-up observations. For each GRB, Tables 1 and 2 present the short and long prompt gamma-ray fluence, X-ray afterglow flux at eleven hours, and optical $R$-band magnitude at eleven hours (times here are measured in the observer's frame). We also include basic properties of the burst: the satellite and frequency range of the prompt data and the redshift, if known. The data come from over 700 unique sources; these sources are listed in the tables. We thoroughly searched the literature to provide the most accurate values. Only the first page of Table 2 is presented in the printed version of this paper. The entire table can be found in the Electronic Supplement. 
The decision of whether a burst is classified as short or long is based upon many factors in the prompt emission and not merely on the burst duration. When a burst's sub-type is questionable, we adopt the classification published by the instrument team. Formal measures of $\mathrm{T}_{90}$ do not always accurately distinguish long from short, such as in cases where we observe a short hard spike followed by a long, soft tail of emission (e.g. GRB 050724 or GRB 051227; Barthelmy et al. 2005a,b). Some bursts, notably GRB 040924 and GRB 050925, have short durations but soft emission, hence are likely the tail-end of the long-duration population (Huang et al. 2005; Holland et al. 2005). GRB 050911 has a complex BAT $\gamma$-ray light curve that may be interpreted as short, but because it is not conclusively in either category (Page et al. 2006a), it is not included.

Our primary sources for GRB fluence and duration are published catalogues, such as those for Swift (Sakamoto et al. 2008) or HETE (Sakamoto et al. 2005), however some are taken from refereed papers or, if no other source is available, from GCN Circulars. If no error is given in the original publication, we assume that the GRB is detected at the $3 \sigma$ level. Because this data set includes bursts discovered by many satellites, we list in tables 1 and 2 the frequency range in which the prompt emission is observed.

All fluences have been transformed into the Swift 15-150 keV fluence range by determining empirical transformations derived from bursts that were detected by multiple satellites. For all bursts with detections by more than one satellite, we calculate the fluence ratio between the two detectors, and the deviation about this ratio. Due to sparse data and lack of overlap in mission lifetimes, in many instances it is necessary to perform multiple transformations to arrive at the final Swift BAT fluence (e.g. using the Ulysses to Swift ratio in order to relate INTEGRAL and Swift). However, the additional scatter introduced in doing so is included in the final ratio error. We jointly calculate the transformation for the similar HETE 25-100 keV, Ulysses 25-100 keV, and BATSE 20-100 keV bands, and find the ratio to Swift to be $1.65 \pm 0.31$. The high-energy Konus-Wind (typically 20-2000 or 20$10000 \mathrm{keV}$ ) and RHESSI (30-10000 keV) fluences were similarly grouped and found to have a rough transformation ratio of $0.37 \pm 0.23$ into the Swift range. Few bursts (seven) had joint detections with INTEGRAL (20-200 keV); these indicate a ratio of $0.95 \pm 0.68$. The HETE $(30-400 \mathrm{keV})$ ratio is calculated to be $0.63 \pm 0.32$, and the BeppoSAX ratio is $0.67 \pm 0.45$. The scaling and large additional errors introduced by this method compensate for the additional scatter caused by comparing bursts between different detectors. If we restrict our analysis to Swift bursts only, our results do not significantly change.

X-ray fluxes, corrected for Galactic extinction, are primarily taken from large-scale uniform studies that fit light curves to estimate the flux at eleven hours, such as those for Swift (Gehrels et al. 2008) or BeppoSAX (de Pasquale et al. 2006) GRBs. For bursts not 
included in these studies, we obtained data from refereed papers, the Swift XRT Lightcurve Repository (Evans et al. 2007), or GCN Circulars. Nearly all X-ray afterglow fluxes are given in the $0.3-10 \mathrm{keV}$ range, however a few were reported in a different energy range and these are noted in Tables 1 and 2. For the few bursts with no reported Galactic extinction, we estimated the extinction from the neutral hydrogen column density using the Chandra Colden and PIMMS online calculators. These bursts are noted in Tables 1 and 2. In a few instances where no error is given, we assume that the source is detected at the $3 \sigma$ level. We choose detections or limits as close to eleven hours as possible that we then extrapolated using temporal slopes of $\alpha_{S, X}=-1.22 \pm 0.37$ and $\alpha_{L, X}=-1.17 \pm 0.30$ for SGRBs and LGRBs respectively. These slopes are averages derived from power-law fits that we performed of the light curves of all bursts observed by XRT until December $31^{s t} 2007$ that have a welldefined temporal slope based on data that extend from minutes until at least eleven hours after the burst. Figure 1 presents a histogram of these slopes and corresponding Gaussian least-squares fit to the scatter in the population. The original measurement error and the error associated with the extrapolation are added in quadrature to determine the final error reported in the tables.

Optical magnitudes are obtained from refereed papers when available and from GCN Circulars when not. We chose observations taken as close to eleven hours as possible, preferably in the $R$-band. All optical magnitudes have been corrected for Galactic extinction using the method presented in Schlegel et al. (1998). If no error is given, as is often the case for GCN derived magnitudes, we again assume that the source is detected at the $3 \sigma$ level. These data are extrapolated in time using a temporal slope of $\alpha_{L, o p t}=-0.85 \pm 0.35$ for long and $\alpha_{S, \text { opt }}=-0.68 \pm 0.20$ for short GRBs, and in frequency using a spectral slope of $\beta=-1.0$. Because of a lack of early optical data, the optical indices are averages based on all afterglow observations with data that extend from at least three hours until after eleven hours following the burst. We fit power-laws to the afterglows of the five SGRBs and 81 LGRBs that fit this selection criteria. Figure 2 presents a histogram of these slopes and Gaussian least-squares fit to the scatter. The original measurement error and the error associated with the extrapolation are added in quadrature to determine the final error.

Often, multiple optical limiting magnitudes exist for a given GRB, and in that case, we took care to choose the one that is most constraining. This sometimes can be arbitrary since the level of constraint depends upon the intrinsic shape of the light curve and redshift of the progenitor, both of which are unknowns given a non-detection. At early times, optical afterglows are often not simple power-laws so we avoided limiting magnitudes taken in the first few minutes. Additionally, we require that $>95 \%$ of the satellite-localized error circle be covered by optical observations, and we assume that the limiting magnitude of the DSS is $R=20$. 
The computed average optical indices are significantly shallower than the X-ray indices. One may expect an observational bias to be introduced because we may be preferably selecting afterglows with shallower temporal decays, because afterglows are typically more difficult to observe in the optical than in the X-ray. In order to show that our analysis is unbiased, we made the following test. We selected X-ray indices by the criteria of the GRB having an optical detection, and through this, we applied the same optical selection effect to the X-ray. When making this selection, we found that the average X-ray temporal slopes remain nearly unchanged ( -1.19 versus -1.16$)$. Therefore, we conclude that the difference between the optical and X-ray temporal indices is real and is not an observational effect.

\section{Data Analysis}

The entire sample of 421 long and 37 short bursts are presented in Tables 1 and 2 and are plotted in Figures 3 and 4. Figure 3 presents the optical brightness at eleven hours (observer frame) versus the prompt high-energy fluence; Figure 4 presents the X-ray brightness at eleven hours (observer frame) versus the prompt fluence. In both figures, we see the same trend: GRBs with high fluence or energy have greater brightness in both optical and X-ray. Not unexpectedly, at both high and low afterglow wavelengths, there is a large intrinsic scatter in the data; the full width in the optical distribution spans nearly four orders of magnitude. A portion of the scatter is certainly intrinsic to the population - we do not expect tight linear correlations. However, the scatter in the optical is larger than in the $\mathrm{X}$-ray, and this may be partially due to observational effects. The optical data comes from a wider variety of sources: different telescopes, filters, instruments, observing teams, reduction and calibration methods, etc. Line-of-sight source-frame absorption may also play a role in the large optical scatter.

The 126 long and 15 short bursts with measured redshift are presented in the rest-frame in Figures 5 and 6. Figure 5 plots the optical, while Figure 6 plots the X-ray luminosities versus the prompt $\gamma$-ray energy, $E_{\gamma, \mathrm{ISO}}$. As discussed above, these plots exhibit a clear correlation between the afterglow brightness at eleven hours and the prompt GRB fluence (note that in figures 5, 6] and 7) the time is measured in the source frame, and thus is redshift corrected with respect to the observed time). Eight of the short bursts have redshifts derived from hosts found with accurate optical positions or are associated with Abell clusters, while seven have hosts determined by XRT error circles only. These seven bursts are differentiated from the bursts with secure redshifts in Figures 5 and 6, but are included in the fit detailed below. In calculating the isotropic equivalent energy emitted in $\gamma$-rays, $\mathrm{E}_{\gamma, \mathrm{ISO}}=4 \pi F_{\gamma} d_{L}^{2}(1+$ $z)^{-1}$, the luminosity distance, $d_{L}$ is determined assuming $H_{o}=71 \mathrm{~km} \mathrm{~s}^{-1} \mathrm{Mpc}^{-1}, \Omega_{M}=0.27$, 
$\Omega_{\Lambda}=0.73$. We transformed the optical data from observer frame filters to rest-frame $R$ band using an assumed spectral slope of $\beta=-1$. The unabsorbed X-ray fluxes have been transformed to $5 \mathrm{keV}$ in the source frame assuming the integrated flux has a spectral slope of $\beta=-1$ between $0.2-10 \mathrm{keV}$ in the observer frame.

While the existence of correlations for each of the two populations by themselves is not surprising, it is clear from Figures 5 and 6 that the distributions of the long and short bursts over-lap in both prompt and afterglow energies. In afterglow brightness, both the observed and rest-frame populations overlap such that for a given fluence, the two classes are not significantly different. In each instance, both in optical and X-ray, and in observer and restframe, the afterglow brightnesses scale roughly as a power-law with energy. Therefore, we model the data as a one-dimensional Gaussian distribution about a straight line in log-log space: $\log \left(F_{R, X}\right)=a+\alpha \log \left(E_{\gamma, \text { ISO }}\right)$. We take into account errors in both coordinates by minimizing the $\chi^{2}$ merit function:

$$
\chi^{2}(a, \alpha)=\sum_{i=1}^{n} \frac{\left(y_{i}-a-\alpha x_{i}\right)^{2}}{\sigma_{y i}^{2}+\alpha^{2} \sigma_{x i}^{2}}
$$

where we have fit to the line $y(x)=a+\alpha x$. We note that physically, $y(x) \equiv \log \left(F_{R, X}\right)$, $x \equiv \log \left(E_{\gamma, \text { ISO }}\right)$, and $\sigma_{x i}$ and $\sigma_{y i}$ are the $x$ and $y$ standard deviations for the $i$ th point. The errors alone over-constrain the linear fit and produce poor $\chi^{2}$ merit values. Therefore, in order to compensate for the intrinsic width of the distribution, we add a constant $1 / 3$ dex error to each error bar to mimic the scatter effect. Increasing the assumed population width past $1 / 3$ dex does not significantly affect the final best fits. Our fits using this technique can be found in Table 3. The slopes and intercepts of the long and short burst distributions are equivalent, within our ability to fit them. While the small number of short bursts means that the errors on a combined slope and intercept fit are large, if we restrict the slope to $\alpha=1$, then the intercepts (i.e. the brightnesses) of the two distributions are found to be the same well within the error of $\sim 0.2$ dex.

The X-ray sample is dominated by observations performed by Swift, and therefore provides a largely homogeneous sample for our analysis. However, the interpretation of the optical data is hindered by the vast diversity of follow-up programs and typical depths of searches. Many optical limits exist for bursts which do not constrain the distribution, while some are deeply constraining. Without knowing the intrinsic source-frame extinction, it is not possible to determine whether these reflect the intrinsic scatter in the distribution or if they are a result of line-of-sight effects. Limits of X-ray afterglows are rare and often due to a delayed follow-up program rather than intrinsic dimness of the afterglows. Therefore, we do not fit to limits in our analysis in either optical or X-ray. However, ignoring limits may introduce a selection bias to the fits. 
Figures 3 and 4 plot observer-frame quantities and therefore have not been corrected for distance. Given a broadly homogeneous population, we expect the effect of distance to dominate the slope of the populations. In a Euclidean universe we would expect the slope imposed to be $\alpha=1$. The real universe, of course, is expanding, and there is a factor of $1+z$ difference between the correction of a bolometric luminosity and a flux. However, if, as is frequently found, the spectral slope of the afterglow is $\beta \sim-1$, this factor of $1+z$ is largely canceled by the k-correction. And this may largely be responsible for the excellent consistency between the populations seen in the Figures and Table 3.

In Figures 5 and 6 we show the subset of bursts with published redshifts. Compared to the previous plots, the scatter in both figures has been reduced. It is clear from both figures, that the dominant predictor of afterglow brightness is the prompt energy emission. The fits to the optical and X-ray flux, given in Table 4 are quite consistent between long and short bursts. If we again assume that the afterglow luminosities scale directly with energy, and impose a slope of $\alpha=1$ on the rest-frame distributions, we find that the long bursts are only brighter on average than the short bursts for a given $E_{\gamma, \text { ISO }}$ by about a factor of two, while the long bursts themselves vary in brightness in the X-ray and optical by orders of magnitude for a given $E_{\gamma, \text { ISO }}$. However, this difference is only at the $\sim 1 \sigma$ level and thus may be entirely statistical rather than real. We note here that similar results to those discussed so far have been derived by two other groups working contemporaneously, Gehrels et al. (2008) and Kann et al. (2009).

Readers may be concerned by the fact that we have not corrected the Swift data for the loss of total fluence in the $\gamma$-ray due to the rather low high-energy cutoff of the Swift BAT $150 \mathrm{keV}$. This is well below the peak of the spectral energy distribution of most short bursts, although it is above the peak for most LGRBs with $E_{\gamma, \text { ISO }} \lesssim 10^{52.5}$ ergs (Amati 2006). Thus we may be underestimating the true $\gamma$-ray fluence of the short burst compared to the long bursts in some cases by up to a factor several (Kann et al. 2009), particularly at low values of $E_{\gamma, \text { ISO }}$ where the two types of bursts overlap. However, this correction would affect the plots of the afterglow flux density in the optical and X-ray versus $E_{\gamma, \text { ISO }}$ similarly. As we will show in the next section, it is the ratio of the optical afterglow flux density to that in the X-ray which provides this paper's real insight into the density of the media surrounding the bursts, and this ratio is unaffected by corrections to $E_{\gamma}$ or $E_{\gamma, \text { ISO }}$. 


\section{A Comparison of Theory with Observations}

\subsection{The Standard Model}

In order to compare the results presented in the previous section to the predictions of the standard model of GRB afterglow emission, we first give a short description of the model's predictions (For a much more detailed introduction to the theory see, e.g., Sari et al. 1998). Afterglow emission follows the propagation of a relativistic shock wave that moves through the cold ambient medium, whose density is assumed constant, $n=1 n_{0} \mathrm{~cm}^{-3}$. A canonical value of $n_{0}=1$ is often used in the literature for LGRBs, but values fitted from afterglow light curves and spectra of long GRBs range from $\sim 10^{-3}$ to $\sim 10^{2}$ (e.g., Panaitescu \& Kumar 2002; Soderberg et al. 2006a). The shock wave moves in a self-similar motion (Blandford \& McKee 1976), which implies shocked fluid Lorentz factor $\Gamma(E, n, R)=\left(17 E / 16 \pi n m_{p} c^{2} R^{3}\right)^{1 / 2}$. Here, $E \equiv E_{\mathrm{ISO}}$ is the total explosion energy 1 , and $R$ is the radius of the shock wave, which is related to the observed time via $t \approx R / 4 \Gamma^{2} c$ (Waxman 1997).

The shock wave accelerates electrons and generates magnetic field. The accelerated electrons assume power law distribution $n_{e l}(\gamma) d \gamma \propto \gamma^{-p} d \gamma$ above $\gamma_{m}=\epsilon_{e}\left(m_{p} / m_{e}\right) g(p) \Gamma$. Here, $\epsilon_{e}$ is the fraction of post-shock thermal energy that goes into the electrons, $\Gamma$ is the Lorentz factor of the shocked fluid, and $g(p)$ is a function of the power law index of the accelerated electrons, $g(p)=(p-2) /(p-1)$ for $p>2, g(p)=\log \left(\gamma_{\max } / \gamma_{m}\right)^{-1} \approx 1 / 7$ for $p=2$, where $\gamma_{\max }$ is the maximum Lorentz factor in which power law distribution of the electrons exist (e.g., Pe'er \& Waxman 2005). A fraction $\epsilon_{B}$ of the post shock thermal energy is assumed to be converted to the magnetic field. With these definitions, synchrotron flux peaks at observed frequency

$$
\nu_{m}^{o b}=\frac{3}{4 \pi} \Gamma \gamma_{m}^{2} \frac{q B}{m_{e} c}=7.8 \times 10^{12} g(p)^{2}(1+z)^{1 / 2} E_{52}^{1 / 2} \epsilon_{e,-1}^{2} \epsilon_{B,-2}^{1 / 2} t_{d}^{-3 / 2} \mathrm{~Hz},
$$

where $Q=10^{x} Q_{x}$ in cgs units was used, and the observed time is measured in days.

The peak of the observed spectral flux at $\nu_{m}$ is

$$
F_{\nu, \text { max }}^{o b}=\frac{1}{4 \pi d_{L}^{2}} N_{e} P_{\nu_{m}} \approx \frac{N_{e}}{4 \pi d_{L}^{2}} \frac{P_{t o t}}{\nu_{m}^{o b}}=7.3(1+z) \mathrm{d}_{\mathrm{L}, 28}^{-2} \mathrm{E}_{52} \epsilon_{\mathrm{B},-2}^{1 / 2} \mathrm{n}_{0}^{1 / 2} \mathrm{mJy},
$$

where $P_{t o t}=\Gamma^{2}(4 / 3) c \sigma_{T} \gamma_{m}^{2}\left(B^{2} / 8 \pi\right)$ is the total synchrotron power radiated by electrons at $\gamma_{m}, \sigma_{T}$ is Thomson cross section, and $N_{e}=4 \pi R^{3} n / 3$ is the total number of radiating electrons. In estimating the observed flux, luminosity distance $d_{L}=10^{28} d_{L, 28} \mathrm{~cm}$ is assumed.

\footnotetext{
${ }^{1}$ Note the difference between the total explosion energy $E_{\mathrm{ISO}}$, and the energy emitted in $\gamma$-rays, $E_{\gamma, \text { ISO }}$.
} 
The synchrotron peak flux frequency is below the break frequency $\nu_{c}$, the characteristic synchrotron emission frequency of electrons for which the synchrotron cooling time, $6 \pi m_{e} c / B^{2} \sigma_{T} \gamma_{c}(1+Y)$ is comparable to the ejecta (rest frame) expansion time, $\sim r / \Gamma c$,

$$
\nu_{c}^{o b}=4.0 \times 10^{15}(1+Y)^{-2}(1+z)^{-1 / 2} E_{52}^{-1 / 2} \epsilon_{B,-2}^{-3 / 2} n_{0}^{-1} t_{d}^{-1 / 2} \mathrm{~Hz} .
$$

Here, $Y$ is the Compton parameter. As we show below, for the canonical parameters values used here, after 11 hours $Y \ll 1$. Therefore, the inclusion of $Y$ does not change the parametric dependence of $\nu_{c}$.

The results in equation 2 indicate that the peak frequency is below the optical band $\left(\nu_{R}=5 \times 10^{14} \mathrm{~Hz}\right)$ after 11 hours, even for equipartition values of $\epsilon_{e}$ and $\epsilon_{B}$. Since $\nu_{c}>\nu_{m}$, (the "slow cooling" regime), the flux at frequencies above $\nu_{m}$ is given by

$$
F_{\nu}^{o b}= \begin{cases}F_{\nu, \max }^{o b}\left(\nu / \nu_{m}\right)^{-(p-1) / 2}, & \nu_{m}<\nu<\nu_{c} \\ F_{\nu, \max }^{o b}\left(\nu_{c} / \nu_{m}\right)^{-(p-1) / 2}\left(\nu / \nu_{c}\right)^{-p / 2}, & \nu_{c}<\nu\end{cases}
$$

\subsection{The Comparison}

Two of the primary observational results obtained in this paper, summarized in figures 5 and 6, can now be compared to the predictions in equations 2 - 5. For bursts with known redshift, $E_{\text {ISO }}$ and observed time $t_{d}=11 / 24$, adopting the assumption that $2 \leq p \leq 2.5$, there are 3 free parameters whose values are unknown: the ambient medium number density $n$ and the parameters $\epsilon_{e}$ and $\epsilon_{B}$. In order to remove some of the degeneracy, we look at the ratio of the fluxes at the $R$ and the $X$ bands. This ratio for the long and short GRBs is presented in Figure 7 .

While the peak flux frequency is below the optical $(\mathrm{R})$ band, the value of the cooling break depends on the density of the ambient medium and on $\epsilon_{B}$. Thus, three options exist:

1. The break frequency is above the observed X-ray frequency, $\nu_{R}<\nu_{X}=1.25 \times 10^{18} \mathrm{~Hz}<$ $\nu_{\mathrm{c}}^{\mathrm{ob}}$. In this case the ratio of the fluxes at the $\mathrm{R}$ and $\mathrm{X}$-ray bands is predicted by equation 5 to be $F_{R} / F_{X}=\left(\nu_{x} / \nu_{R}\right)^{(p-1) / 2}=50$ (for $\mathrm{p}=2$ ). This ratio is inconsistent with the results presented in Figure [7, which clearly indicate a ratio $F_{R} / F_{X} \simeq 1-2 \times 10^{3}$ for both the short and long bursts. The flux ratio increases with $p$, and for $p=2.5$ it is

$\sim 350$. Still, this is lower than that observed for the vast majority of bursts, and is more than an order of magnitude below the ratio of many.

2. The break frequency is in between the optical and the X-ray bands, $\nu_{R}<\nu_{c}^{o b}<\nu_{X}$. As noted earlier many studies of afterglows from long GRBs have found this to be 
the case (e.g. Galama et al. 1998b; Harrison et al. 1999; Panaitescu \& Kumar 2002; Soderberg et al. 2006a). Indeed, in this situation, the expected ratio of the fluxes, $F_{R} / F_{X}=\left(\nu_{x} / \nu_{R}\right)^{(p-1) / 2}\left(\nu_{x} / \nu_{c}\right)^{1 / 2}=720 E_{52}^{1 / 4} \epsilon_{B,-2}^{3 / 4} n_{0}^{1 / 2}$ (calculated for $\nu_{c}$ after 11 hours and for $p=2)$ is consistent with the results obtained in Figure 7 for the long bursts. For $p=2.5$, this ratio is somewhat higher $\approx 5 \times 10^{3} E_{52}^{1 / 4} \epsilon_{B,-2}^{3 / 4} n_{0}^{1 / 2}$, which is slightly above the results in Figure 7 .

On the other hand, for characteristic energy $E \simeq 10^{50}$ erg typical for the short bursts, this ratio is $F_{R} / F_{x} \simeq 130 E_{50}^{1 / 4} \epsilon_{B,-2}^{3 / 4} n_{0}^{1 / 2}$ (for $p=2$ ), nearly an order of magnitude lower than the result obtained in Figure 7 for the short bursts. For $p=2.5$, the obtained value is $F_{R} / F_{x} \simeq 1600 E_{50}^{1 / 4} \epsilon_{B,-2}^{3 / 4} n_{0}^{1 / 2}$, consistent with the results in this figure. The value of $F_{R} / F_{X} \approx 1600$ is obtained for values of the ambient number density of short bursts which are comparable to that expected for long bursts, $n_{0}=1$. Lower values of the number density would be inconsistent with the results in Figure 7 , unless the value of $\epsilon_{B}$ is larger for short bursts than for the long ones. This discrepancy is further aggravated by observed $F_{R} / F_{x}$ values around $10^{4}$ for several short bursts. It is hard to make these consistent with the theory using any set of parameters consistent with standard expectations.

3. The third possibility is that the cooling frequency is below the $\mathrm{R}$ band, i.e., $\nu_{c}^{o b}<$ $\nu_{R}<\nu_{X}$. Using equation 5 one obtains the ratio of the $\mathrm{R}$ to $\mathrm{X}$-ray fluxes to be $F_{R} / F_{X}=\left(\nu_{x} / \nu_{R}\right)^{p / 2}=2500$ (for $p=2$ ), which is consistent with the results presented in Figure 7. On the other hand, the requirement that $\nu_{c}^{o b}<\nu_{R}$ for short GRBs for which $E=10^{30} \mathrm{erg}$ implies $n_{0}>130 \epsilon_{B,-2}^{-3 / 2}$. Even for equipartition value of the magnetic field this requirement implies value of $n$ which is at least as high for short GRBs as for the long GRBs.

The analysis presented above implies that under the assumption of the synchrotron emission model, the characteristic values of the number densities of short GRBs are required to be at least similar to the characteristic values obtained for long GRBs, $n \gtrsim 1 \mathrm{~cm}^{-3}$. Lower values of the number density in short bursts require stronger magnetic field. Moreover, the results hint toward power law index $p \simeq 2.5$ in short bursts.

The ratios calculated above are done in the framework of the standard model, which assumes knowledge of total explosion energy $E_{\mathrm{ISO}}$, while for bursts with measured redshift we only measure the energy emitted in $\gamma$ rays, $E_{\gamma, \text { ISO }}$. However, as we show in $\$ 4.3$ below, the efficiency in converting the explosion energy into $\gamma$ rays is very high, $E_{\gamma, \mathrm{ISO}} / \epsilon_{e} E_{\mathrm{ISO}} \simeq 1$ for both the long and short bursts. We note that in principle it is possible that the average values of the microphysical parameter $\epsilon_{e}, \epsilon_{B}$ and the power law index $p$ are different for short and long GRBs, which may affect our result. However, we find this possibility unlikely, since 
after 11 hours both types of bursts are well in the self-similar phase, in which the properties of the blast wave - that determines the values of these parameters - are well defined.

The basic results found do not change if one assumes that the flux at the X-ray band is dominated by Compton scattering (see, e.g., Sari \& Esin 2001; Zhang \& Mészáros 2001). For $\nu_{c}^{o b}<\nu_{X}$ and $\nu_{m}^{o b} \gamma_{m}^{2}<\nu_{X}<\nu_{c}^{o b} \gamma_{m}^{2}$, one obtains the ratio of the Compton to the synchrotron flux at the X-band to be $F_{X, I C} / F_{X, s y n} \approx(16 / 3) \sigma_{T} n r \gamma_{m}^{p-1}\left(\nu_{x} / \nu_{c}\right)^{1 / 2}=2.8 \times$ $10^{-2} g(p) E_{52}^{5 / 8} \epsilon_{e,-1} \epsilon_{B,-2}^{3 / 4} n_{0}^{9 / 8} t_{d}^{1 / 8}$, where $p=2$ is assumed. This ratio clearly indicates that inverse Compton emission does not dominate the flux at the $\mathrm{X}$ band unless the number density is significantly higher than $n_{0}=1$. Therefore, addition of IC component does not ease the requirement for high values of the number density in short GRBs.

We have so far assumed that the density of the ambient medium is constant, i.e. independent of radius from the burst. This, however, may not be the case. Motivated by the association of long GRBs to the deaths of massive stars, some authors (c.f. Woosley 1993; Paczynski 1998) have proposed that the explosion producing a GRB may occur into a wind, ejected by the progenitor prior to its explosion. This scenario results in an ambient matter density profile, $\rho(r)=A r^{-2}$, with characteristic value $A=5 \times 10^{11} A_{*} \mathrm{gr} \mathrm{cm}^{-2}$ typical for Wolf-Rayet star (Chevalier \& Li 2000).

In an explosion into density profile, the evolution of the Lorentz factor of the flow in its similar expansion phase is $\Gamma(E, r)=\left(9 E / 16 \pi \rho c^{2}\right)^{1 / 2} r^{-3 / 2}$, and the relation between the radius of the shock front and the observed time is $t \approx r / 2 \Gamma^{2} c$ (Pe'er \& Wijers 2006). Similar calculations to the ones presented in equations 2-4 results in (see, e.g., Panaitescu \& Kumar 2001)

$$
\begin{aligned}
& \nu_{m}^{o b .}=1.6 \times 10^{11} g(p)^{2}(1+z)^{1 / 2} E_{52}^{1 / 2} \epsilon_{e,-1}^{2} \epsilon_{B,-2}^{1 / 2} t_{d}^{-3 / 2} \mathrm{~Hz}, \\
& \nu_{c}^{o b .} \quad=1.4 \times 10^{14}(1+Y)^{-2}(1+z)^{-3 / 2} E_{52}^{1 / 2} A_{*}^{-2} \epsilon_{B,-2}^{-3 / 2} t_{d}^{1 / 2} \mathrm{~Hz}, \\
& F_{\nu, \max }^{o b} \simeq 45(1+z) \mathrm{d}_{\mathrm{L}, 28}^{-2} \mathrm{E}_{52}^{1 / 2} \mathrm{~A}_{*} \epsilon_{\mathrm{B},-2}^{1 / 2} \mathrm{t}_{\mathrm{d}}^{-1 / 2} \mathrm{mJy} .
\end{aligned}
$$

Focusing on the case $\nu_{m}^{o b}<\nu_{R}<\nu_{c}^{o b}<\nu_{X}$, using similar arguments to the ones presented for the constant density scenario discussed above imply that the observed ratio $F_{R} / F_{X} \simeq 10^{3}$ is obtained for combination of the parameters which fulfill $E_{52}^{1 / 2} A_{*}^{-2} \epsilon_{B,-2}^{-3 / 2} \approx 33$. For long GRBs characterized by $E \simeq 10^{52} \mathrm{erg}$ and canonical value of $\epsilon_{B}=0.01$, the obtained value of $A_{*}$ is $A_{*} \approx 0.15$. For short GRBs for which $E \approx 10^{49} \mathrm{erg}$, the obtained value is lower, $A_{*} \lesssim 0.03$. The assumption $\nu_{c}^{o b}>\nu_{X}$ is inconsistent with the results presented in Figure 7 and the assumption $\nu_{c}^{o b}<\nu_{R}$ leads to higher values of $A_{*}$.

We thus find it possible to explain the results found for short GRBs in a scenario in which the explosion that produces short GRBs occurs into a wind profile, with wind density which is $\sim 30$ times lower than the typical value for Wolf-Rayet stars. There are both strong theoretical (c.f. Chevalier \& Li 2000) and observational (Starling et al. 2008) 
reasons to believe that a substantial fraction of long GRBs would explode into a medium with a wind structure. However, the standard models of SGRB production involve the merger of compact binaries. The mass loss from the supernovae producing the compact remnants should, however, impart velocities to the binaries that would take them far outside of any remnant stellar winds long before they merge. Therefore, if a wind density profile is indeed a key to understanding the emission of SGRBs, a novel progenitor system will be required.

\subsection{A Further Limit on Emission Mechanisms}

The results presented here also place strong constraints on the efficiency of $\gamma$-ray production during prompt emission, significantly extending the limits derived in earlier works (Freedman \& Waxman 2001; Berger et. al. 2003). For $\nu_{c}^{o b}<\nu_{X}$ and assuming power law index $p=2$, equations 2 - 5 predict the flux at the X-ray band after 11 hours to be $F_{X}^{o b} \simeq 0.3(1+z) \mathrm{d}_{\mathrm{L}, 28}^{-2} \mathrm{E}_{52} \epsilon_{\mathrm{e},-1} \mu \mathrm{Jy}$. This result depends only on the energy in the lepton component, which is a fraction $\epsilon_{e}$ of the total explosion energy $E$. For bursts with known luminosity distance, the rest-frame brightness is thus

$$
F_{\nu, X} \simeq 3 \times 10^{26} E_{52} \epsilon_{e,-1} \mathrm{erg} \mathrm{s}^{-1} \mathrm{~Hz}^{-1}
$$

(note that an order unity uncertainty may exist due to the approximations used in deriving the equations, as well as the assumption that $p=2$ ). Comparison with the results presented in Table 4 and Figure 6 shows that for a given X-ray brightness, the energy emitted in $\gamma$ rays is similar to the energy carried by the leptons. Thus, we can conclude that the $\gamma$ ray production is efficient, $E_{\gamma, \mathrm{ISO}} / \epsilon_{e} E_{\mathrm{ISO}} \simeq 1$. This result holds for at least the seven orders of magnitude in energies considered in this work.

The similarity found between the X-ray fluxes of short and long GRBs implies that the high efficiency in $\gamma$ ray production is a property of both types. This result is consistent with the tentative conclusion of Bloom et al. (2006), who studied a single SGRB, namely GRB050509b.

\section{Systematic biases and possible caveats}

Our sample includes total of 37 short GRBs, out of which 16 (43\%) have measured redshift. From those bursts, seven have measured optical and X-ray fluxes at 11 hours and thus the fluence ratio at 11 hours (rest frame) can be obtained, and nine have only upper limits on the observed optical fluence, and thus only upper limits on the ratio at 11 hours

are known (see figures 5, 7). Therefore, a question may arise as to whether our conclusions 
could be biased by selection effects, and in particular, whether bursts in low density regions might be excluded from our sample because their afterglows would be expected to be too weak.

Consider, however, all bursts with $\gamma$-ray fluence larger than $10^{-7} \mathrm{erg} \mathrm{cm}^{-2}$ (see figure 3 and tables 1, 2). Essentially all long GRBs are in this group, as are more than half of the short bursts (22 out of 37). Furthermore, above this fluence, 8 out of 9 short bursts which have reasonably deep $(<3 \mu J y$ or $A B=T B D)$ mag early searches, have identified optical counterparts. Indeed, with one exception, GRB 061210, all the optical upper limits lie above detections with comparable $E_{\gamma}$.

If the standard model is correct and the prompt gamma-ray fluence arises from an internal process, then choosing this sample in no way selects bursts by the properties of their external medium. However, because an optical ID has been necessary to get a secure redshift for a short GRB, this is the prime sample used to derive a relationship between the fluxes $F_{R}, F_{x}$ and $E_{\gamma, \text { ISO }}$ in Figures 5 - 7. Our fits then (in particular Figure 5) depend on a sample which is essentially complete, and which does not depend upon the properties of the external medium, unless the external medium influences the observed $E_{\gamma}$.

Of course, if $E_{\gamma, \text { ISO }}$ depends upon the external medium, and in particular its density, then our conclusions would be biased. But this bias only arises if perhaps the most interesting (and potentially controversial) of our possible conclusions is true: the initial gamma-ray fluence is not entirely due to an internal process but is also regulated by the medium external to the exploding object.

A further comparison of optical and X-ray fluxes is presented in figure 8. In this figure, we plot the ratio of the observed fluxes for bursts, using the full sample of bursts for which detections or upper limits exist. Since the redshift, and thus $E_{\gamma, \text { ISO }}$, is in most cases unknown, we plot a histogram of the optical to X-ray flux ratio at 11 hours observed time. The histogram clearly shows that the two distributions exhibit a Gaussian-like behavior, with very similar means, $\left\langle F_{R} / F_{X}\right\rangle=770$ for the long bursts and $\left\langle F_{R} / F_{X}\right\rangle=1130$ for the short bursts, where only those bursts with optical and X-ray detections (no upper limits) were used in this calculation. The similar Gaussian shapes and similar means of the SGRB and LGRB populations also suggests that contaminations, in particular incorrect identification of a long burst as short, does not significantly affect the distribution (otherwise a bi-modal distribution would likely have been seen - unless, of course both distributions have the same ratio, which is the main conclusion here).

Again, it is the fact that the values $\left\langle F_{R} / F_{X}>\right.$ for the short bursts are near 1000 (and comparable or larger than those of long bursts) which is surprising. It is then interesting 
that three very clear, well studied short bursts, 050709, 050724 and 051221A have values of $<F_{R} / F_{X}>$ of about 7000, 10,000 and 500 respectively. Examination of the table by the reader will show that even if some of the short bursts in our sample are falsely identified short bursts, the bursts which are clearly short agree with the claims presented here.

Additionally, it is possible that the ratio of $f_{R} / f_{X}$ could be affected absorption of photons in the host galaxy. While many long GRBs show substantial X-ray columns Campana et al. (2006); Schady et al. (2007); Galama \& Wijers (2001), the large majority of the observed 10 $\mathrm{keV}$ passband of the XRT is unaffected, and thus our X-ray fluxes should be fairly accurate. The measured optical absorption of long gamma-ray burst afterglows often suggests lower column densities than the X-ray, with typical optical absorptions in the range of one-tenth to one magnitude Schady et al. (2007); Cenko et al. (2009). The effect of host absorption on short bursts has not yet been well studied. But again, the main danger would be a suppression of the optical relative to the X-ray. However, our main result is that the short bursts have brighter optical emission relative to the X-ray than expected by the standard model and typically assumed environmental densities.

\section{Summary and discussion}

In this work, we present a comprehensive study of the optical and X-ray afterglows obtained after 11 hours of 37 short and 421 long GRBs, and compared the results to the energy emitted in $\gamma$-rays during the prompt emission phase. This sample is the largest used so far for this type of study. We find a strong correlation between the optical and X-ray afterglow brightnesses and prompt $\gamma$-ray fluence of GRBs, a result similar to one reported contemporaneously by by Gehrels et al. (2008) Kann et al. (2009). However, we also show that the ratio of the optical to X-ray emission in the short burst afterglows is comparable to that of the long-bursts. This equivalence between burst types, and the absolute ratio we find can only be understood in the framework of the standard theory if the circumburst density of SGRBs is typically comparable to, or even higher than the circumburst density of LGRBs. We point out that in a wind-like scenario for SGRBs can also explain this result if the average mass loss rate of the SGRB progenitor is $\sim 30$ times lower than the characteristic

mass loss rate from a Wolf-Rayet star. Finally, we have show that in the framework of the standard model, in which the prompt emission fluence is independent of the environmental density, by selecting bursts with $\gamma$-ray fluence larger than $10^{-7} \mathrm{erg} \mathrm{cm}^{-2}$ we obtain for short bursts above this fluence an essentially complete sample for analysis.

These results indicate that SGRB afterglows are not necessarily dim due to a lower density in the circumburst environment. While the average SGRB afterglow is significantly 
dimmer than the average LGRB afterglow, this appears to be largely due to the lower total energy release of the burst, as indicated by the fainter prompt $\gamma$-ray emission. We can thus conclude that if the prompt emission is a result of internal shocks, then either the external density of long and short bursts is similar, or the afterglow flux is less sensitive to the density of the surrounding medium than predicted. On the other hand, if the prompt emission is due to an external shock, both quantities the gamma-ray fluence and afterglow intensity will depend on the external density and may scale accordingly. In this case we could attribute the faintness of both the prompt and afterglow emission to the external medium density.

Due to the short lifetimes of the massive star progenitors of long-duration GRBs, they have been traditionally expected to occur in or near giant molecular clouds $\left(n \sim 10^{2.5} \mathrm{~cm}^{-3}\right.$; Shu et al. 1987). Some observational evidence supports this hypothesis. The hosts of LGRBs are typically blue and actively forming massive stars (Fruchter et al. 1999; Sokolov et al. 2001; Le Floc'h et al. 2003; Christensen et al. 2004; Gorosabel et al. 2005). Fruchter et al. (2006) find that LGRBs are preferentially located on the brightest regions of these galaxies, which they interpret as evidence that GRBs frequently occur on or near massive OB associations. X-ray observations of GRB afterglows show neutral hydrogen column densities consistent those of Galactic giant molecular clouds (Galama \& Wijers 2001; Jakobsson et al. 2006; Schady et al. 2007). At optical wavelengths, moderate extinction characteristic of dense regions is seen in some bursts (Kann et al. 2007), while in others the afterglow appears significantly extinguished (Djorgovski et al. 2001; Levan et al. 2006a; Watson et al. 2006; Pellizza et al. 2006; Rol et al. 2007), and at high redshifts where Ly $\alpha$ absorption can be found in the optical spectrum, large hydrogen column densities (up to $10^{23} \mathrm{~cm}^{-3}$ ) are frequently found (c.f. Jakobsson et al. 2007). Nonetheless, as noted earlier, the density of the medium into which the relativistic flow of the GRB expands may be dominated by an earlier wind from the progenitor star rather than the ISM.

In contrast, SGRBs are found in both elliptical and star-forming galaxies, a fact consistent with merger scenarios. The ISM of elliptical galaxies is sparse and has a typical density of only $n \sim 10^{-2.5} \mathrm{~cm}^{-3}$ (Fukazawa et al. 2006). Panaitescu (2006), however, estimates a circumburst density of $10^{-1}<n<10^{3} \mathrm{~cm}^{-3}$ for GRB 050724, a burst in an E/S0 galaxy (Berger et al. 2005). At the same time circumburst densities of $10^{-4}<n<10^{-1} \mathrm{~cm}^{-3}$ Panaitescu (2006) and $10^{-3}$ to $10^{-1} \mathrm{~cm}^{-3}$ Soderberg et al. (2006a) are found for GRBs 050709 and $051221 \mathrm{~A}$, respectively, both of which occurred in apparently star-forming galaxies. As we have shown here, the lower ranges of these two latter densities are inconsistent with the distribution of ratios of $F_{X} / F_{R}$ seen in Figure 7 . These estimates of the SGRB circumburst density, however, assume a locally uniform medium. If the SGRBs were to typically form in a windlike structure, this ratio would be acceptable. Still, the common presence of a wind structure around SGRBs would rule out the standard merger models. 
The velocity by mass loss during the supernovae, let alone any kicks by the supernovae, would easily move the compact remnants by more than 100 pc from their natal region before a binary merger occurred (Belczynski et al. 2006a).

A potentially simple explanation of the results presented here is that both the afterglow and the prompt emission are due to an external interaction (e.g., Dermer 2008). In this case, both the afterglow and prompt emission might be expected to scale similarly. However, it is difficult to explain the strong variability observed in many bursts in such a model (see, e.g., Mészáros 2006). Furthermore, observational evidence for a correlation between the density of a circumburst medium and $\mathrm{E}_{\gamma, \mathrm{ISO}}$ is so far either weak or potentially damaging. Troja et al. (2008) have suggested that SGRBs far from the center of their host may be fainter than those closer in. However, this work uses a number of associations based on the brightest galaxy in a rather large Swift XRT error circle, and therefore may in some cases assign an incorrect offset, and thus will need to be confirmed on larger, better samples. In contrast, the only work to examine this issue for long bursts has found a positive correlation between the brightness of the burst and the distance of the burst from the center of the host (Ramirez-Ruiz et al. 2002). This is, to first order, the opposite of one would predict if the external medium played a role in the gamma-ray emission. It has, however, been noted that the bursts GRBG 050509b and 050724, both of which were in early type galaxies were intrinsically weaker than bursts found in star-forming hosts Rhoads (2009). Nonetheless, we note again that the work by Panaitescu (2006) suggests that the circumburst medium around at GRB 0507024 was reasonably dense.

Several recent advances in the theory and modelling of the binary merger process and the local insterstellar medium could potentially explain our results. van den Heuvel (2007) has pointed out that the large majority of known double neutron-star binaries have low eccentricities, suggesting that the second born neutron stars in binaries, unlike single neutron stars, typically did not receive substantial "kick" velocities at birth. Lower kicks will lead to lower systemic velocities, and a higher probability that the system remains in a moderately dense ISM when the binary merges. Furthermore, the time to merger may often be relatively short. Belczynski et al. (2009) estimate that up to $70 \%$ of NS-NS and NS-BH binaries may merge with $100 \mathrm{Myr}$ after formation. Indeed, one burst that appears to have likely been a short burst, GRB 060505, lies in an [HII] region on the outskirts of its spiral host (Fynbo et al. 2006; Ofek et al. 2007; Levesque \& Kewley 2007). Some short bursts, however, occur in early-type galaxies. Here recent result could also provide an explanation for an apparent ISM density of $1 \mathrm{~cm}^{-3}$. Binary mergers in early-type galaxies are almost certainly dominated by the evolving binaries in globular clusters (Grindlay et al. 2006), and the most rapid evolution is likely to occur in the densest clusters. These clusters however, could have ionized gas densities of the required magnitude due to the colliding winds of giants contained 
in the cluster (Parsons, Ramirez-Ruiz \& Lee 2009).

Although we may not fully understand the mechanism, we cannot escape the conclusion that SGRBs are fundamentally similar in emission properties to LGRBs - particularly the fainter LGRBs that have typically been found at low redshift. It has been suggested that low-luminosity LGRBs (e.g. GRB 980425, GRB 031203 and GRB 060218) have large, and perhaps nearly quasi-spherical, opening angles when compared to traditional LGRBs (Soderberg et al. 2004b, 2006b; Liang et al. 2007). Similarly, of the three SGRBs with estimated opening angles, GRB 050709 and GRB 050724 have large measured angles, $\Theta \sim 14^{\circ}$ (Fox et al. 2005) and $\sim 25^{\circ}$ (Grupe et al. 2006; Malesani et al. 2007) respectively, while GRB 051221 has $4^{\circ}<\Theta<8^{\circ}$ (Burrows et al. 2006; Soderberg et al. 2006a), which is more typical of traditional high $\mathrm{E}_{\gamma, \text { ISO }}$ LGRB opening angles. Yet both long and short populations appear to largely fall on a simple log-linear relationship between afterglow intensity and gamma-ray luminosity over six orders of magnitude in energy. Within this relationship there may be strong hints as to the nature of the progenitors of SGRBs, or perhaps a new more fundamental understanding of the GRB emission mechanism.

This work made use of data supplied by the UK Swift Science Data Centre at the University of Leicester. We gratefully acknowledge J. Racusin, N. Gehrels, D. Burrows, and the Swift team for generously sharing pre-published XRT values. We also thank A. Levan, N. Gehrels, E, Waxman, K. Belczynski, E. Ramirez-Ruiz, E. van den Heuvel, R. Sari and J. Graham for helpful discussions, J. Greiner for his online GRB Table and R. Quimby, E. McMahon and J. Murphy for the GRBlog. AP wishes to acknowledge the support of the Riccardo Giacconi fellowship award of the Space Telescope Science Institute.

\section{REFERENCES}

Amati, L. 2006 MNRAS, 372, 233

Antonelli, L. A., et al. 1999, A\&AS, 138, 435

Barbier, L., et al. 2007, GRB Coordinates Network, 6623, 1

Barthelmy, S. D., et al. 2005a, Nature, 438, 994

Barthelmy, S., Gehrels, N., Norris, J., \& Sakamoto, T. 2005b, GRB Coordinates Network, 4401,1

Belczynski, K., Perna, R., Bulik, T., Kalogera, V., Ivanova, N., \& Lamb, D. Q. 2006a, ApJ, 648,1110 
Belczynski, K., Sadowski, A., Rasio, F. A., \& Bulik, T. 2006b, ApJ, 650, 303

Belczynski, K., Stanek, K. Z., \& Fryer, C. L. 2009, ApJ, submitted astro-ph/0712.3309

Berger, E., Kulkarni, S.R. \& Frail, D.A. 2003, ApJ, 590, 379

Berger, E. 2005, GRB Coordinates Network, 3801, 1

Berger, E., \& Boss, A. 2005, GRB Coordinates Network, 4323, 1

Berger, E., \& Fox, D. 2005, GRB Coordinates Network, 4316, 1

Berger, E., et al. 2005a, Nature, 438, 988

Berger, E. 2006a, GRB Coordinates Network, 5965, 1

Berger, E. 2007a, ApJ, 670, 1254

Berger, E. 2007b, GRB Coordinates Network, 5995, 1

Berger, E., \& Kaplan, D. L. 2007, GRB Coordinates Network, 6680, 1

Berger, E., et al. 2007b, ApJ, 664, 1000

Berger, E., Morrell, N., \& Roth, M. 2007b, GRB Coordinates Network, 7154, 1

Bikmaev, I., et al. 2005a, GRB Coordinates Network, 3797, 1

Blandford, R.D., \& McKee, C.F. 1976, Phys. Fluids, 19, 1130

Bloom, J. S., Kulkarni, S. R., \& Djorgovski, S. G. 2002, AJ, 123, 1111

Bloom, J. S., et al. 2006, ApJ, 638, 354

Bloom, J. S., et al. 2007, ApJ, 654, 878

Burrows, D. N., et al. 2006, ApJ, 653, 468

Campana et al. 2006, A\&A, 449, 61

Castro-Tirado, A. J., et al. 1997, IAU Circ., 6598, 2

Castro-Tirado, A., Alises, M., \& Greiner, J. 2000, GRB Coordinates Network, 870, 1

Castro-Tirado, A. J., Bond, I., Kilmartin, P., de Ugarte Postigo, A., Gorosabel, J., Jelinek, M., \& Yock, P. 2005, GRB Coordinates Network, 3018, 1 
Cenko, S. B., Fox, D. B., \& Price, P. A. 2006, GRB Coordinates Network, 5912, 1

Cenko, S. B., Rau, A., Berger, E., Price, P. A., \& Cucchiara, A. 2007, GRB Coordinates Network, 6664, 1

Cenko, S. B. et al. 2009 ApJ693 1484

Chevalier, R. A., Li, Z.-Y. 2000, ApJ, 536, 195

Christensen, L., Hjorth, J., \& Gorosabel, J. 2004, A\&A, 425, 913

Cobb, B. E. 2006, GRB Coordinates Network, 5935, 1

Connaughton, V., et al. 1997, IAU Circ., 6683, 1

Cucchiara, A., Fox, D. B., Berger, E., \& Price, P. A. 2006, GRB Coordinates Network, 5470, 1

Cucchiara, A., Fox, D. B., Cenko, S. B., Berger, E., Price, P. A., \& Radomski, J. 2007, GRB Coordinates Network, 6665, 1

Cusumano, G., Burrows, D. N., Hunsberger, S., Pagani, C., La Parola, V., \& Mangano, V. 2005, GRB Coordinates Network, 4326, 1

D'Avanzo, P., et al. 2008, in preparation

de Pasquale, M., et al. 2006, A\&A, 455, 813

Dermer, C.d. 2008, ApJ, in press (astro-ph/0703223)

Diercks, A. H., et al. 1998, ApJ, 503, L105

Djorgovski, S. G., et al. 1997, Nature, 387, 876

Djorgovski, S. G., Kulkarni, S. R., Goodrich, R., Frail, D. A., \& Bloom, J. S. 1998, GRB Coordinates Network, 137, 1

Djorgovski, S. G., Kulkarni, S. R., Bloom, J. S., \& Frail, D. A. 1999, GRB Coordinates Network, 289, 1

Djorgovski, S. G., Frail, D. A., Kulkarni, S. R., Bloom, J. S., Odewahn, S. C., \& Diercks, A. 2001, ApJ, 562, 654

Djorgovski, S. G., Bloom, J. S., \& Kulkarni, S. R. 2003, ApJ, 591, L13 
Donaghy, T. Q., et al. 2006, ArXiv Astrophysics e-prints, arXiv:astro-ph/0605570

Eichler, D., Livio, M., Piran, T., \& Schramm, D. N. 1989, Nature, 340, 126

Evans, P. A., et al. 2007, A\&A, 469, 379

Fox, D. B., et al. 2005, Nature, 437, 845

Freedman, D.L., \& Waxman, E. 2001, ApJ, 547, 922

Fruchter, A. S., et al. 1999, ApJ, 519, L13

Fruchter, A. S., et al. 2006, Nature, 441, 463

Fukazawa, Y., Botoya-Nonesa, J. G., Pu, J., Ohto, A., \& Kawano, N. 2006, ApJ, 636, 698

Fynbo, J. P. U. et al. 2006, Nature, 444, 1047

Galama, T., et al. 1997, Nature, 387, 479

Galama, T., et al., 1998a, GRB Coordinates Network, 62, 1

Galama, T., et al., 1998b, ApJ, 500L, 101

Galama, T. J., \& Wijers, R. A. M. J. 2001, ApJ, 549, L209

Gehrels, N., et al. 2005, Nature, 437, 851

Gehrels, N., et al. 2008, ApJ, 189, 1161

Grindlay, J., Portegies Zwart, S. \& McMillan, S., Nature Physics, 2, 116

Golenetskii, S., Aptekar, R., Mazets, E., Pal'Shin, V., Frederiks, D., \& Cline, T. 2007, GRB Coordinates Network, 6671, 1

Gorosabel, J., et al. 1999, A\&AS, 138, 455

Gorosabel, J., et al. 2005, A\&A, 444, 711

Graham, J. F., Fruchter, A. S., Levan, A. J., Nysewander, M., Tanvir, N. R., Dahlen, T., Bersier, D., \& Pe'Er, A. 2007, GRB Coordinates Network, 6836, 1

Groot, P. J., et al. 1997a, IAU Circ., 6616, 2

Groot, P. J. et al. 1997b, GRB Coordinates Network, 17, 1

Groot, P. J., et al. 1998a, ApJ, 493, L27 
Groot, P. J., et al. 1998b, ApJ, 502, L123

Grupe, D., Burrows, D. N., Patel, S. K., Kouveliotou, C., Zhang, B., Mészáros, P., Wijers, R. A. M., \& Gehrels, N. 2006, ApJ, 653, 462

Guidorzi, C., et al. 2005, GRB Coordinates Network, 4356, 1

Guidorzi, C., et al. 2007, GCNR, 77, 1 (2007), 77, 1

Halpern, J. P., Tonnesen, S., Tuttle, S., \& Mirabal, N. 2005a, GRB Coordinates Network, 4202, 1

Harrison, F. A., et al. 1999, ApJ, 523, L121

Hjorth, J., et al. 2002, ApJ, 576, 113

Hjorth, J., et al. 2003a, Nature, 423, 847

Hjorth, J., et al. 2005, Nature, 437, 859

Holland, S. T., Barthelmy, S., Beardmore, A., Gehrels, N., Kennea, J., Page, K., Palmer, D., \& Rosen, S. 2005, GRB Coordinates Network, 4034, 1

Holland, S. T., de Pasquale, M., \& Markwardt, C. B. 2007, GRB Coordinates Network, 7145,1

Huang, K. Y., et al. 2005, ApJ, 628, L93

Hurley, K., Cline, T., Frontera, F., Guidorzi, C., Montanari, E., Mazets, E., \& Golenetskii, S. 2000a, GRB Coordinates Network, 895, 1

Hurley, K., Cline, T., Mazets, E., \& Golenetskii, S. 2000b, GRB Coordinates Network, 865, 1

Hurley, K., Cline, T., Mazets, E., \& Golenetskii, S. 2001, GRB Coordinates Network, 916, 1

Hurley, K., et al. 2002a, GRB Coordinates Network, 1409, 1

Hurley, K., et al. 2002b, GRB Coordinates Network, 1719, 1

Jakobsson, P., et al. 2006, A\&A, 460, L13

Jakobsson, P., et al. 2007, A\&A, 406, 13

Johnson, S., et al. 2007, GRB Coordinates Network, 6218, 1 
Kann, D. A., et al. 2007, ArXiv e-prints, 712, arXiv:0712.2186

Kann, D. A., et al. 2008, ApJ, submitted (arXiv:0804.1959)

Kocevski, D., \& Bloom, J. S. 2007, GRB Coordinates Network, 7107, 1

Lamb, D. Q., et al. 2002, GRB Coordinates Network, 1403, 1

Le Floc'h, E., et al. 2003, A\&A, 400, 499

Lee, W. H., Ramirez-Ruiz, E., \& Granot, J. 2005, ApJ, 630, L165

Levan, A., et al. 2006a, ApJ, 647, 471

Levan, A. J., et al. 2006b, ApJ, 648, L9

Levan, A. J., et al. 2007, GRB Coordinates Network, 6603, 1

Levan, A. J., et al. 2008, MNRAS, 384, 541

Levesque, E. M., \& Kewley, L. J., 2007 ApJ667, 121

Liang, E., Zhang, B., Virgili, F., \& Dai, Z. G. 2007, ApJ, 662, 1111

Malesani, D., et al. 2007, A\&A, 473, 77

Marshall, F. E., Takeshima, T. 2000, GRB Coordinates Network, 58, 1

Marshall, F. E., Barthelmy, S. D., Burrows, D. N., Chester, M. M., Cummings, J., Evans, P. A., Roming, P., \& Gehrels, N. 2007a, GCNR, 80, 1 (2007), 80, 1

Marshall, F. E., Barthelmy, S. D., Brown, P. J., Burrows, D. N., Cummings, J., Roming, P., Starling, R., \& Gehrels, N. 2007b, GCNR, 81, 1 (2007), 81, 1

McBreen, S., et al. 2007, GCNR, 46, 2 (2007), 46, 2

Mészáros, P. 2006, Rep. Prog. Phys., 69, 2259

Mészáros, P., \& Rees, M.J. 1997, ApJ, 476, 232

Metzger, M. R., Djorgovski, S. G., Kulkarni, S. R., Steidel, C. C., Adelberger, K. L., Frail, D. A., Costa, E., \& Frontera, F. 1997, Nature, 387, 878

Mineo, T., Tagliaferri, G., Malesani, D., Giommi, P., Burrows, D., Fox, D., Chincarini, G., \& Page, K. 2005, GRBCoordinates Network, 4195, 1 
Mirabal, N., Halpern, J. P., Gotthelf, E. V., \& Mukherjee, R. 2005, ApJ, 620, 379

Murakami, T., Ueda, Y., Yoshida, A., Kawai, N., Marshall, F. E., Corbet, R. H. D., \& Takeshima, T. 1997, IAU Circ., 6732, 1

Nakar, E. 2007, Phys. Rep., 442, 166

Narayan, R., Paczynski, B., \& Piran, T. 1992, ApJ, 395, L83

Nysewander, M., Moran, J., Johnson, L., Moschler, D., Reichart, D., \& Henden, A. 2002, GRB Coordinates Network, 1760, 1

Ofek, E. O.. et al. 2007, ApJ, 66211290

Paciesas, W. S., et al. 1999, ApJS, 122, 465

Paczyński, B. 1998, ApJ, 494, L45

Pagani, C., La Parola, V., \& Burrows, D. N. 2005, GRB Coordinates Network, 3934, 1

Page, K. L., et al. 2006a, ApJ, 637, L13

Panaitescu, A. 2006, MNRAS, 367, L42

Panaitescu, A. \& Kumar, P. 2000, ApJ, 543, 66

Panaitescu, A. \& Kumar, P. 2002, ApJ, 571, 779

Panaitescu, A., Kumar, P., \& Narayan, R. 2001, ApJ, 561, L171

Parson, R. K., Ramirez-Ruiz, E. \& Lee, W. H., 2009, ApJ, submitted (astro-ph/0904.1768)

Pe'er, A., \& Waxman, E. 2005, ApJ, 633, 1018

Pe'er, A., \& Wijers, R.A.M.J. 2006, ApJ, 643, 1036

Pellizza, L. J., et al. 2006, A\&A, 459, L5

Perley, D. A., Thoene, C. C., \& Bloom, J. S. 2007a, GRB Coordinates Network, 6774, 1

Perri, M., Stratta, G., Fenimore, E., Schady, P., Barthelmy, S. D., Burrows, D. N., Roming, P., \& Gehrels, N. 2007a, GCNR, 103, 1 (2007), 103, 1

Piranomonte, S., Covino, S., Malesani, D., Tagliaferri, G., Chincarini, G., \& Stella, L. 2006, GRB Coordinates Network, 5392, 1 
Piranomonte, S., Vergani, S., D’Avanzo, P., \& Tagliaferri, G. 2007, GRB Coordinates Network, 6612,1

Perley, D. A., Bloom, J. S., Modjaz, M., Poznanski, D., \& Thoene, C. C. 2007, GRB Coordinates Network, 7140, 1

Price, P. A., Axelrod, T. S., \& Schmidt, B. P. 2000, GRB Coordinates Network, 898, 1

Price, P. A., Morrison, G., \& Bloom, J. S. 2001a, GRB Coordinates Network, 919, 1

Price, P. A., Axelrod, T. S., Schmidt, B. P., \& Reichart, D. E. 2001b, GRB Coordinates Network, 1020, 1

Price, P. A., Schmidt, B. P., \& Axelrod, T. S. 2002, GRB Coordinates Network, 1410, 1

Price, P. A., Berger, E., Fox, D. B., Cenko, S. B., \& Rau, A. 2006, GRB Coordinates Network, 5077, 1

Ramirez-Ruiz, E., Lazzati, D., \& Blain, A. 2002, ApJ, 565, 9

Reichart, D. E., et al. 1999, ApJ, 517, 692

Rhoads, J. 2009, ApJ, in press (astro-ph/0807.2642)

Rol, E., et al. 2007, ApJ, 669, 1098

Sakamoto, T., et al. 2005, ApJ, 629, 311

Sakamoto, T., et al. 2008, ApJS, 175, 179

Sari, R., \& Esin, A. 2001,ApJ, 548, 787

Sari, R., Piran, T., \& Narayan, R. 1998, ApJ, 497, L17

Schady, P., et al. 2007, MNRAS, 377, 273

Schlegel, D. J., Finkbeiner, D. P., \& Davis, M. 1998, ApJ, 500, 525

Shu, F. H., Adams, F. C., \& Lizano, S. 1987, ARA\&A, 25, 23

Sokolov, V. V., et al. 2001, A\&A, 372, 438

Soderberg, A., Djorgovski, S. G., Halpern, J. P., \& Mirabal, N. 2004a, GRB Coordinates Network, 2837, 1

Soderberg, A. M., et al. 2004b, Nature, 430, 648 
Soderberg, A. M., et al. 2006a, ApJ, 650, 261

Soderberg, A. M., et al. 2006b, Nature, 442, 1014

Stanek, K. Z., et al. 2003, ApJ, 591, L17

Starling, R., Osborne, J. P., Page, K. L., \& Marshall, F. E. 2007, GRB Coordinates Network, 6852,1

Starling, R. L. C., et al. 2008, A\&A, 481,319

Stratta, G., et al. 2007a, A\&A, 461, 485

Stratta, G., et al. 2007b, A\&A, 474, 827

Stratta, G., Perri, M., Conciatore, M., Burrows, D., \& Sato, G. 2007c, GRB Coordinates Network, 6119, 1

Tinney, C., et al. 1998, IAU Circ., 6896, 1

Troja, E., King, A. R., O'Brien, P. T., Lyons, N., \& Cusumano, G. 2008, MNRAS, L5

van den Heuvel, E. P. J. 2007, AIP Conf. Proceedings, 924, 598 (astro-ph/0704.1215)

Villasenor, J. S., et al. 2005, Nature, 437, 855

Vrba, F. J., et al. 2000, ApJ, 528, 254

Vreeswijk, P. M., et al. 1999, ApJ, 523, 171

Watson, D., et al. 2006, ApJ, 652, 1011

Waxman, E. 1997, ApJ, 491, L19

Wiersema, K., et al. 2008, in preparation

Woosley, S. 1993, ApJ, 405, 273

Woosley, S. E., \& Bloom, J. S. 2006, ARA\&A, 44, 507

Xin, L. P., Zhai, M., Qiu, Y. L., Wei, J. Y., Hu, J. Y., Deng, J. S., Urata, Y., \& Zheng, W. K. 2007, GRB Coordinates Network, 6747, 1

Zhang, B., \& Mésáros, P. 2001, ApJ, 559, 110

Zheng, W. K., et al. 2007, GRB Coordinates Network, 6250, 1 
Ziaeepour, H., Barthelmy, S. D., Parsons, A., Page, K. L., de Pasquale, M., \& Schady, P. 2007a, GCNR, 74, 2 (2007), 74, 2 


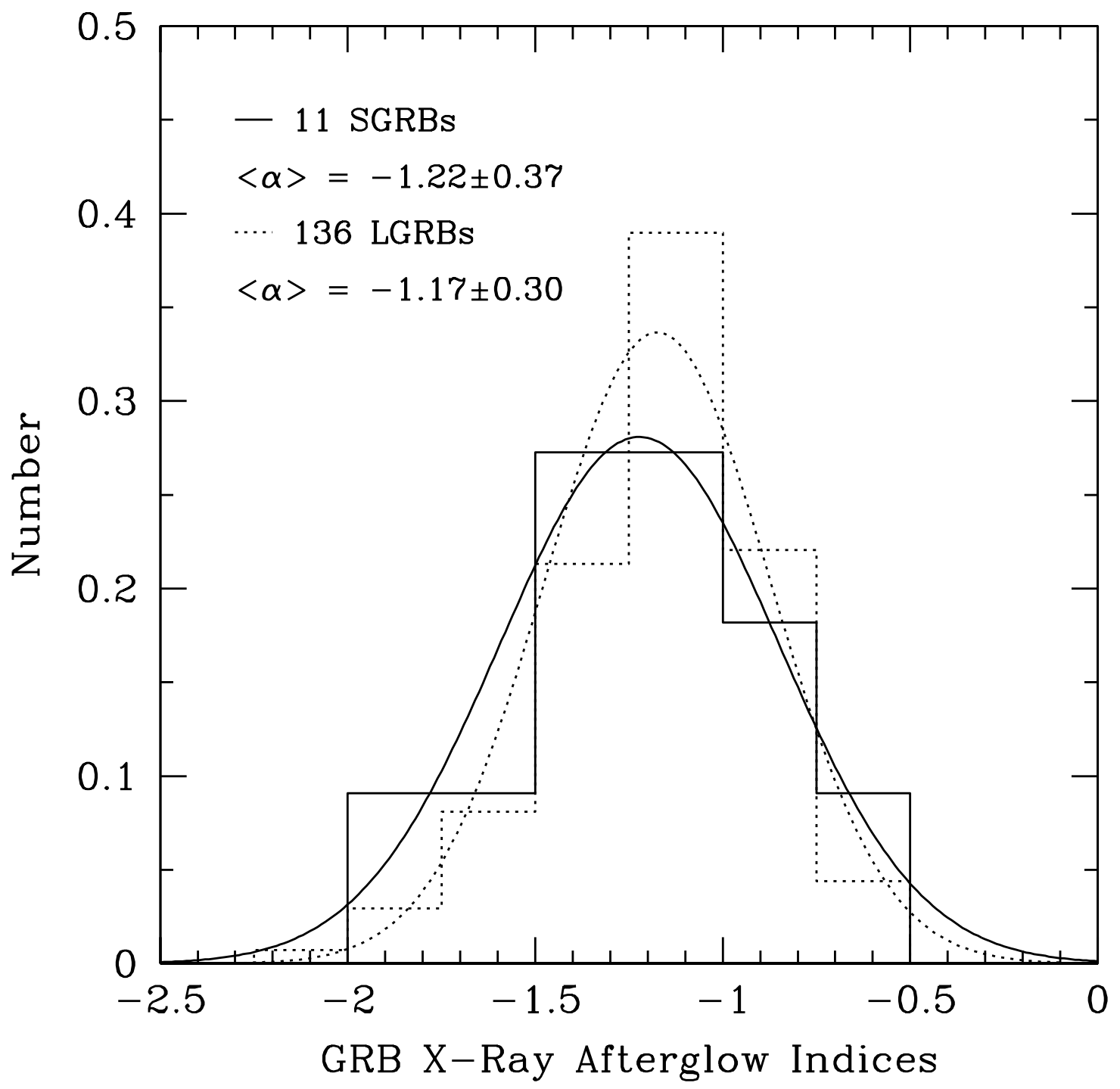

Fig. 1. - A compilation of the X-ray afterglow temporal indices of the two populations and presents the best-fit Gaussian distribution to each data set. We include only afterglow indices of bursts with a rapid XRT detection and with data extending to at least 11 hours. The solid line indicates SGRBs; the dashed indicates LGRBs. The plot has been normalized by the sum of the bursts used: 11 short and 136 long bursts. 


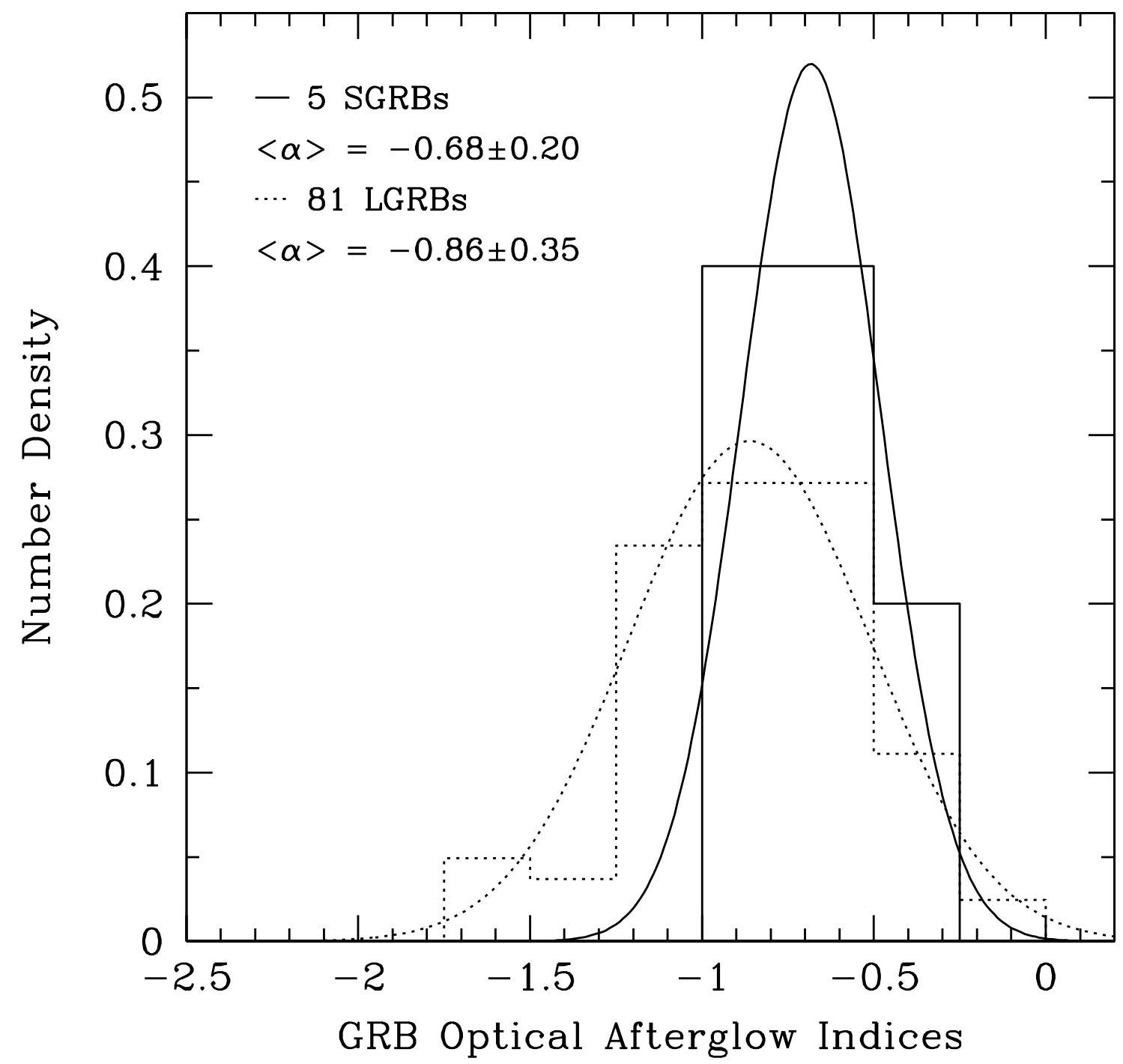

Fig. 2.- A compilation of all short and long burst optical afterglow indices and the best Gaussian fits to the distributions. We include only afterglow data that have light curves extending from at most three hours to at least eleven hours. The solid line indicates SGRBs; the dashed indicates LGRBs. The plot has been normalized by the sum of bursts used: 5 short and 81 long. 


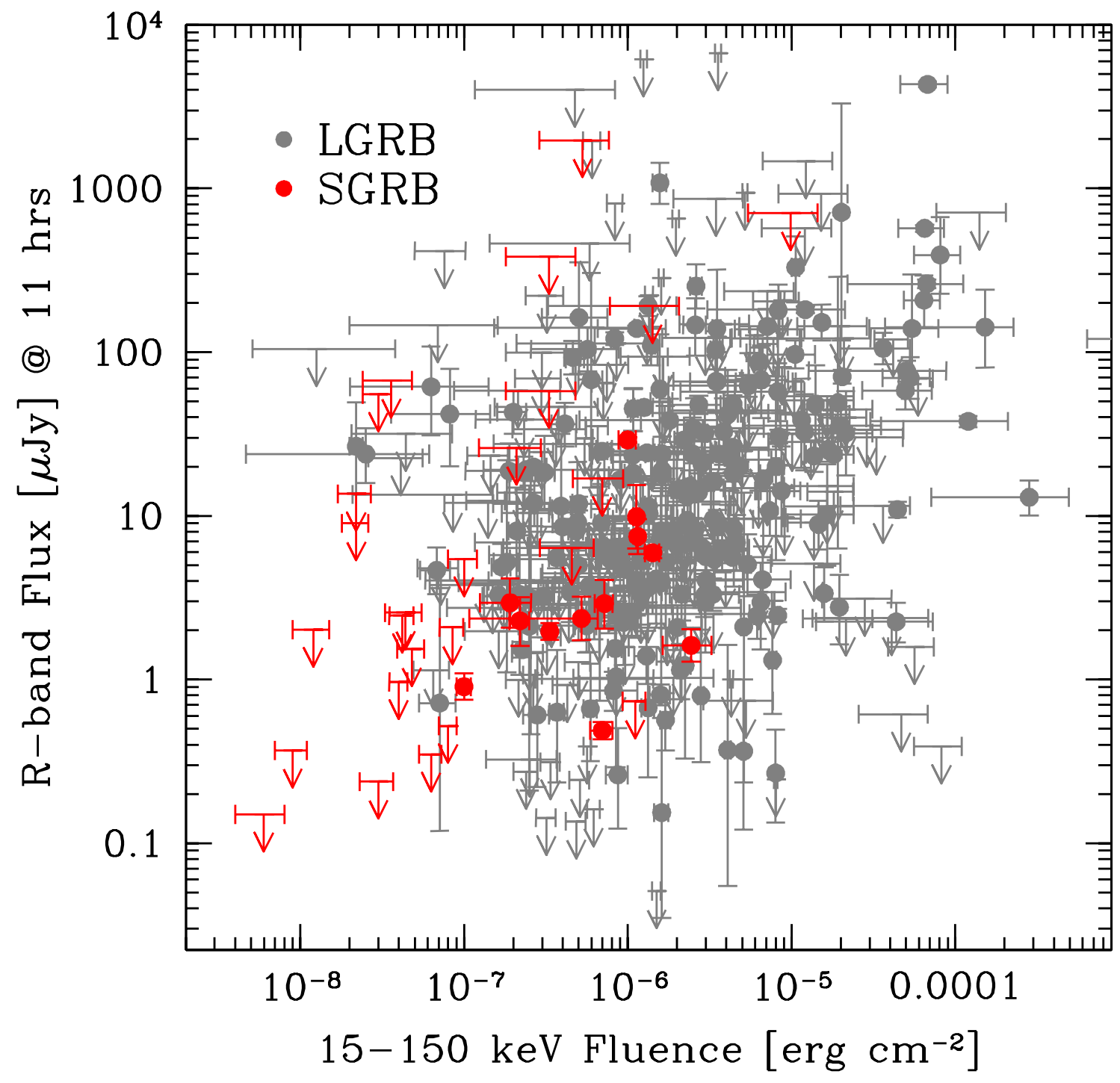

Fig. 3.- A plot of the optical $R$-band flux (corrected for Galactic extinction) at eleven hours (observed frame) versus prompt $15-150 \mathrm{keV} \gamma$-ray fluence for both long (grey) and short (red) bursts. Note that below a fluence of $10^{-7} \mathrm{ergs} \mathrm{cm}^{-2}$, no optical afterglow of an SGRB has been discovered, while above $10^{-7}$, all reasonably deep observing campaigns but one (GRB 061210) have detected an optical afterglow. 


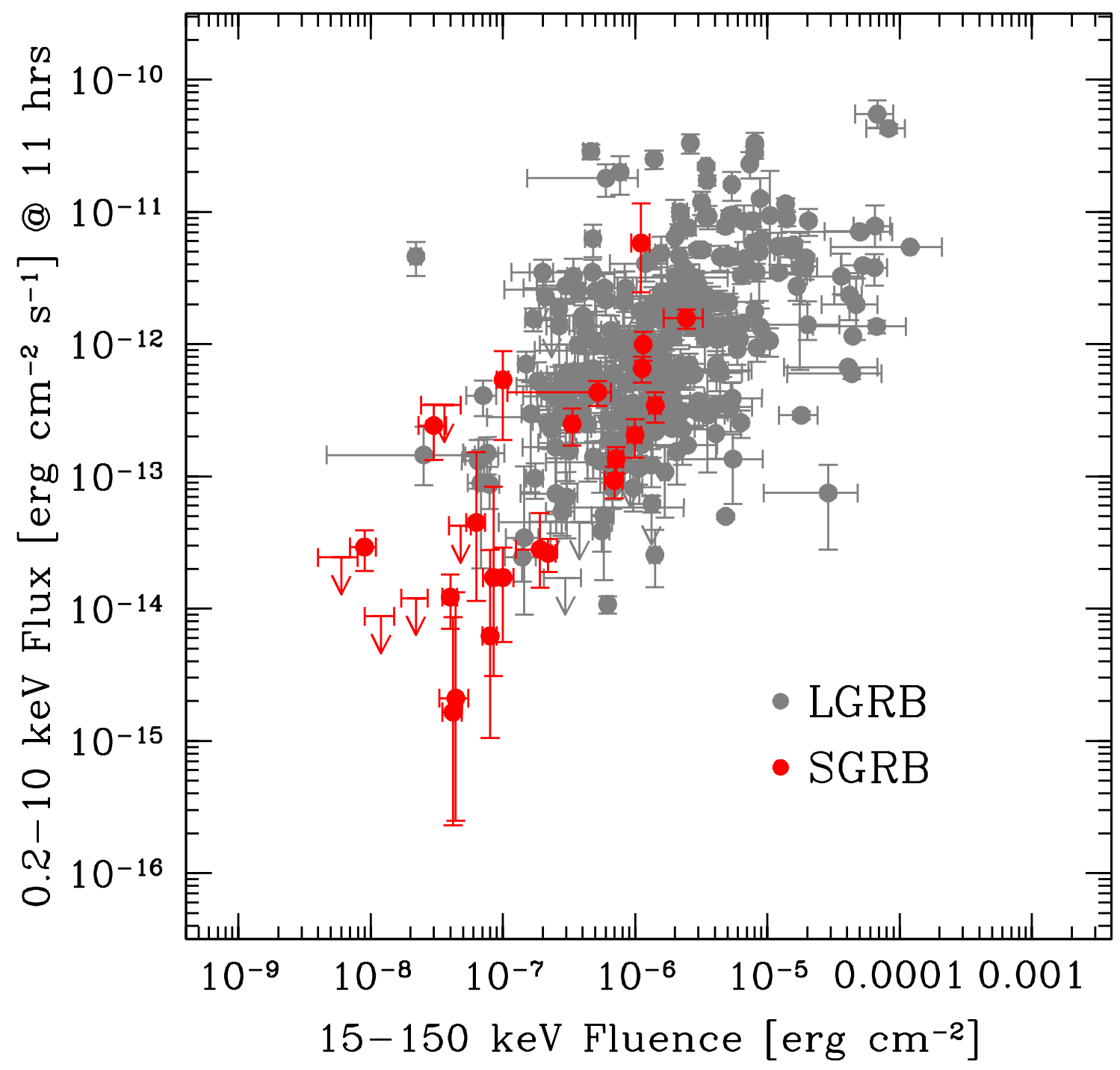

Fig. 4.- A plot of the X-ray flux at eleven hours (observed frame) versus the prompt 15-150 $\mathrm{keV} \gamma$-ray fluence for both long (grey) and short (red) bursts. 


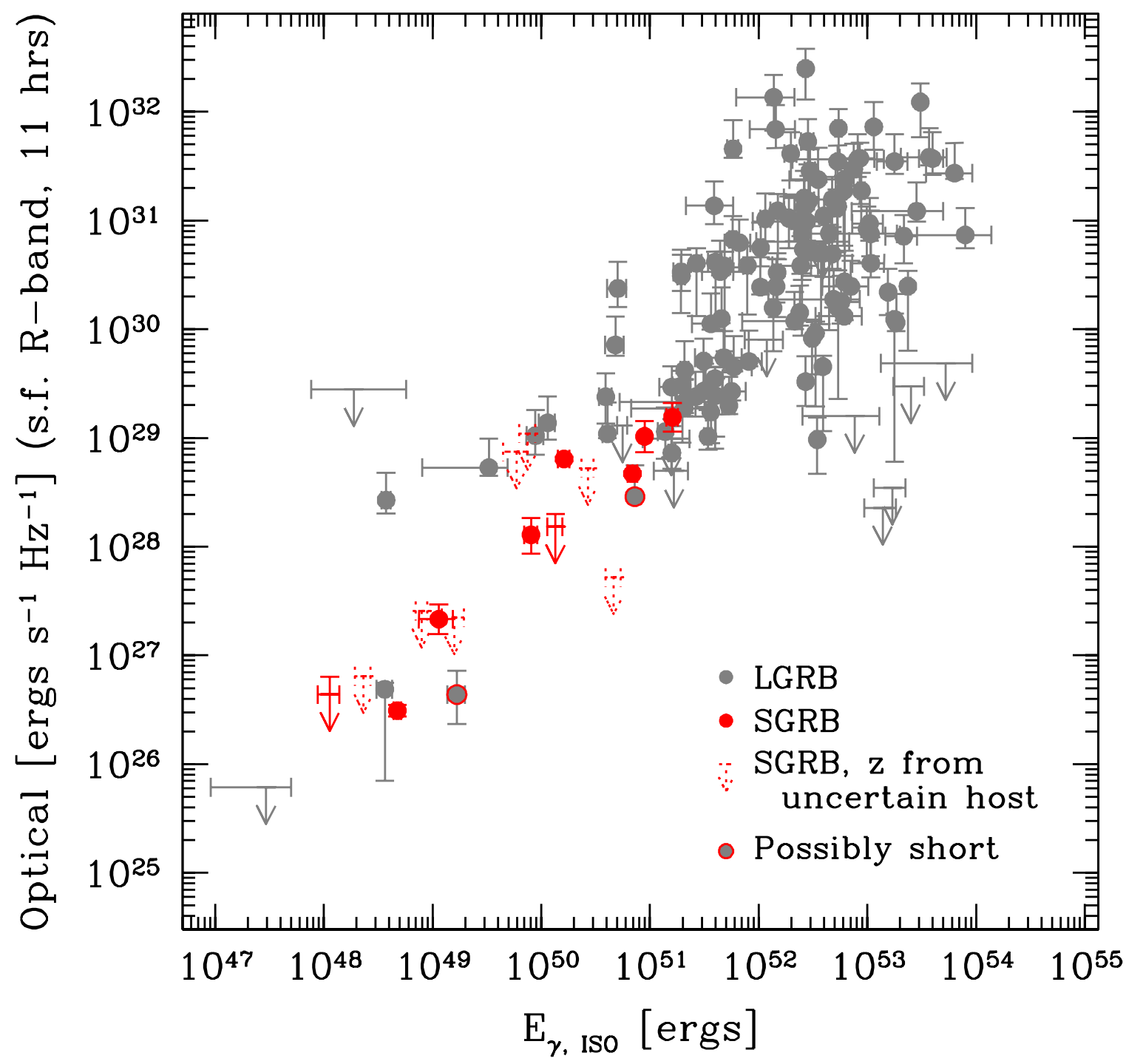

Fig. 5.- A plot of the rest-frame optical $R$-band (corrected for Galactic extinction) afterglow brightness at eleven hours (in the source frame) versus $\mathrm{E}_{\gamma, \mathrm{ISO}}$, the total prompt emission of the burst in gamma-rays. Dashed upper limits represent SGRBs with a host galaxy determined by XRT error circle only. The classification of GRB 060614 and GRB 060505 is uncertain, therefore they are labeled as "Possibly short". 


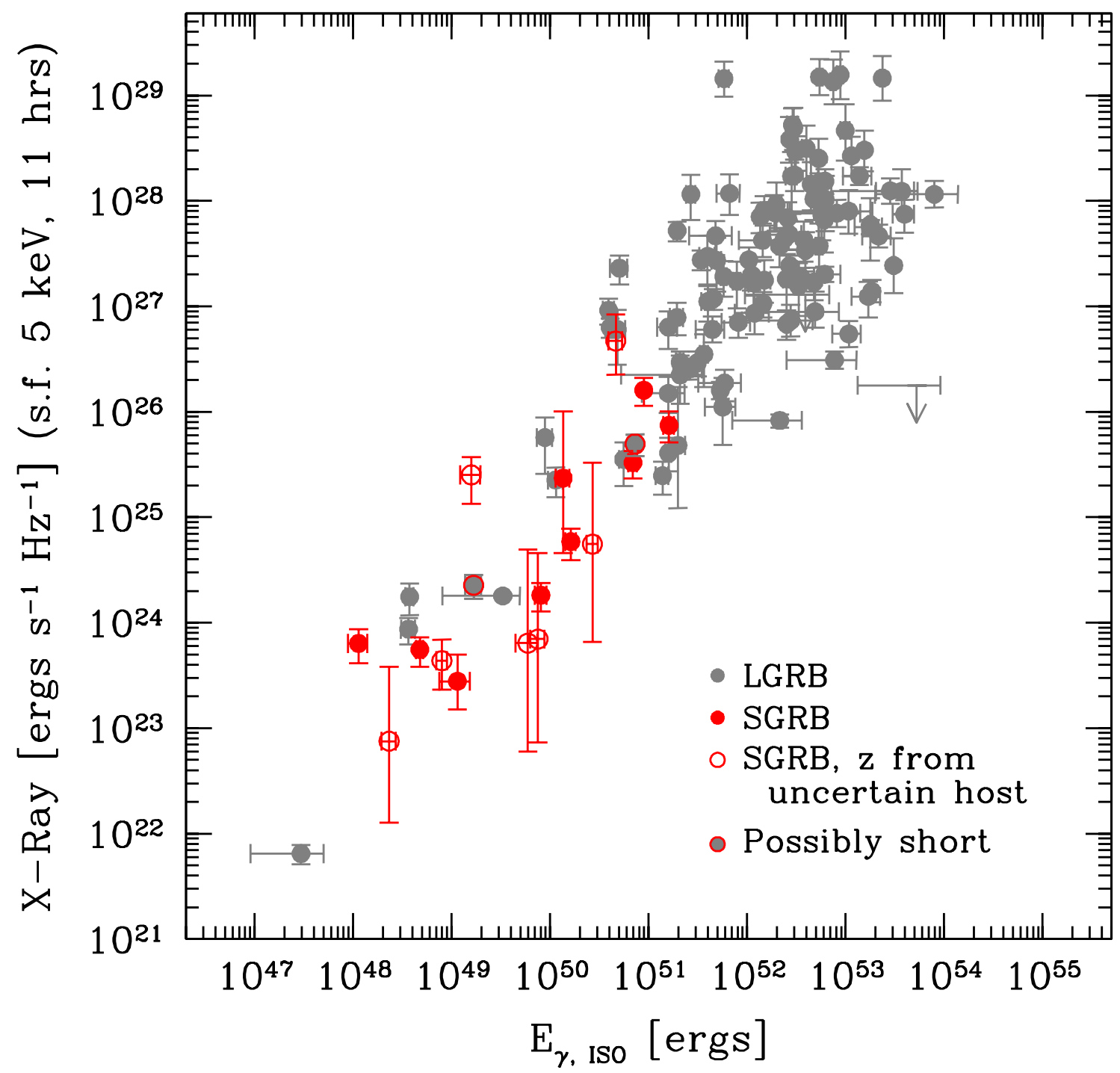

Fig. 6.- A plot of the rest-frame $5 \mathrm{keV}$ X-ray afterglow brightness at eleven hours (rest frame) versus $\mathrm{E}_{\gamma, \mathrm{ISO}}$. The open circles represent $\mathrm{SGRBs}$ with a host galaxy determined by XRT error circle only. The classification of GRB 060614 and GRB 060505 is uncertain, therefore they are labeled as "Possibly short." 


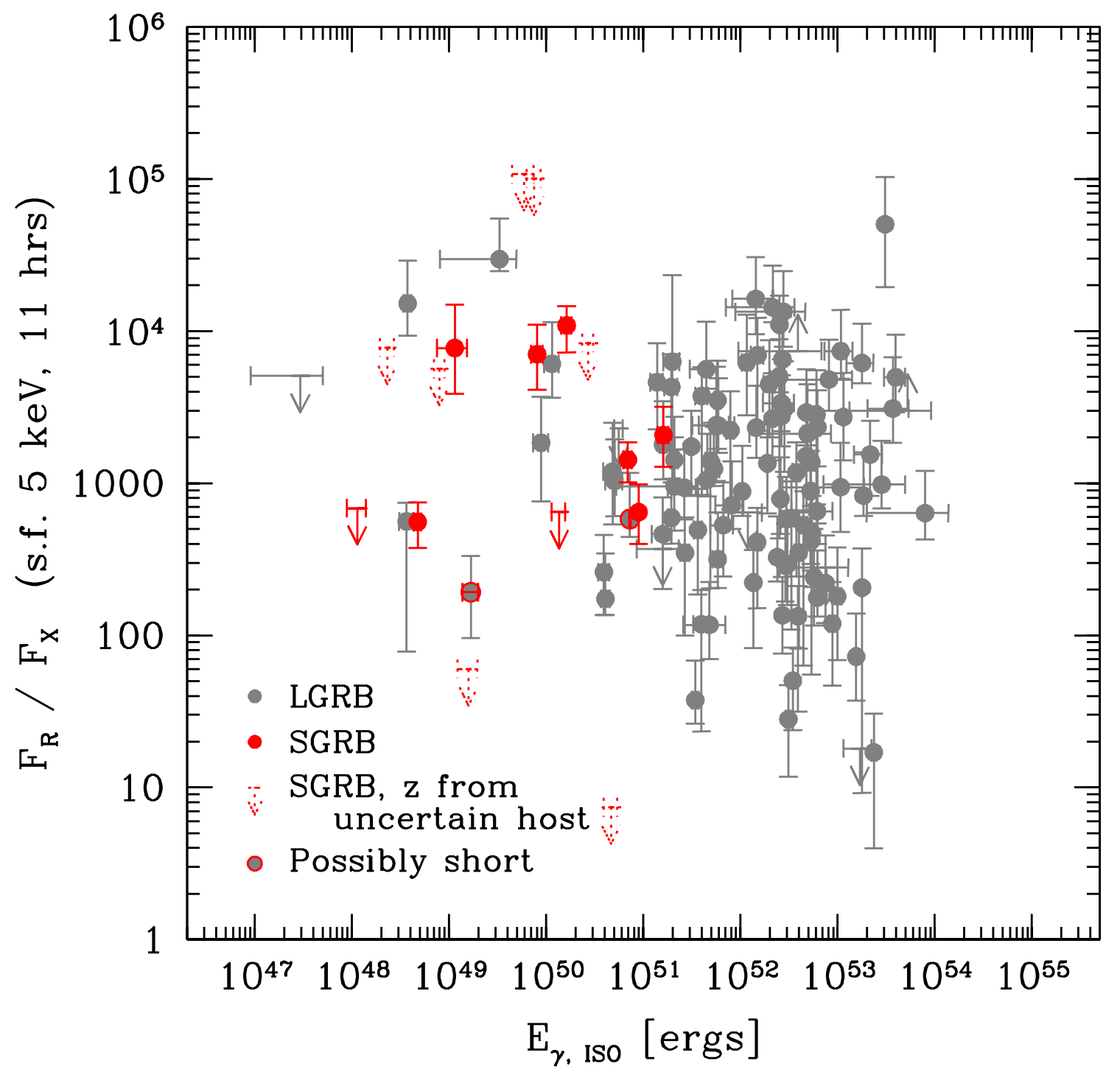

Fig. 7.- The ratio of the total fluence in the optical over that in the $\mathrm{x}$-ray versus the $\mathrm{E}_{\gamma, \mathrm{ISO}}$, measured at 11 hours (source frame). Once again the short and long bursts do not appear to differ, except in their typical $\mathrm{E}_{\gamma, \mathrm{ISO}}$. There is no evidence in a suppression of this ratio either by a low density medium surrounding the short bursts or their lower typical $\mathrm{E}_{\gamma, \mathrm{ISO}}$, as would be expected from the standard theory if the synchroton frequency of the bursts were typically between the optical and X-ray. However, the observed absolute values of this ratio are hard to explain by the standard theory if this is not the case. 


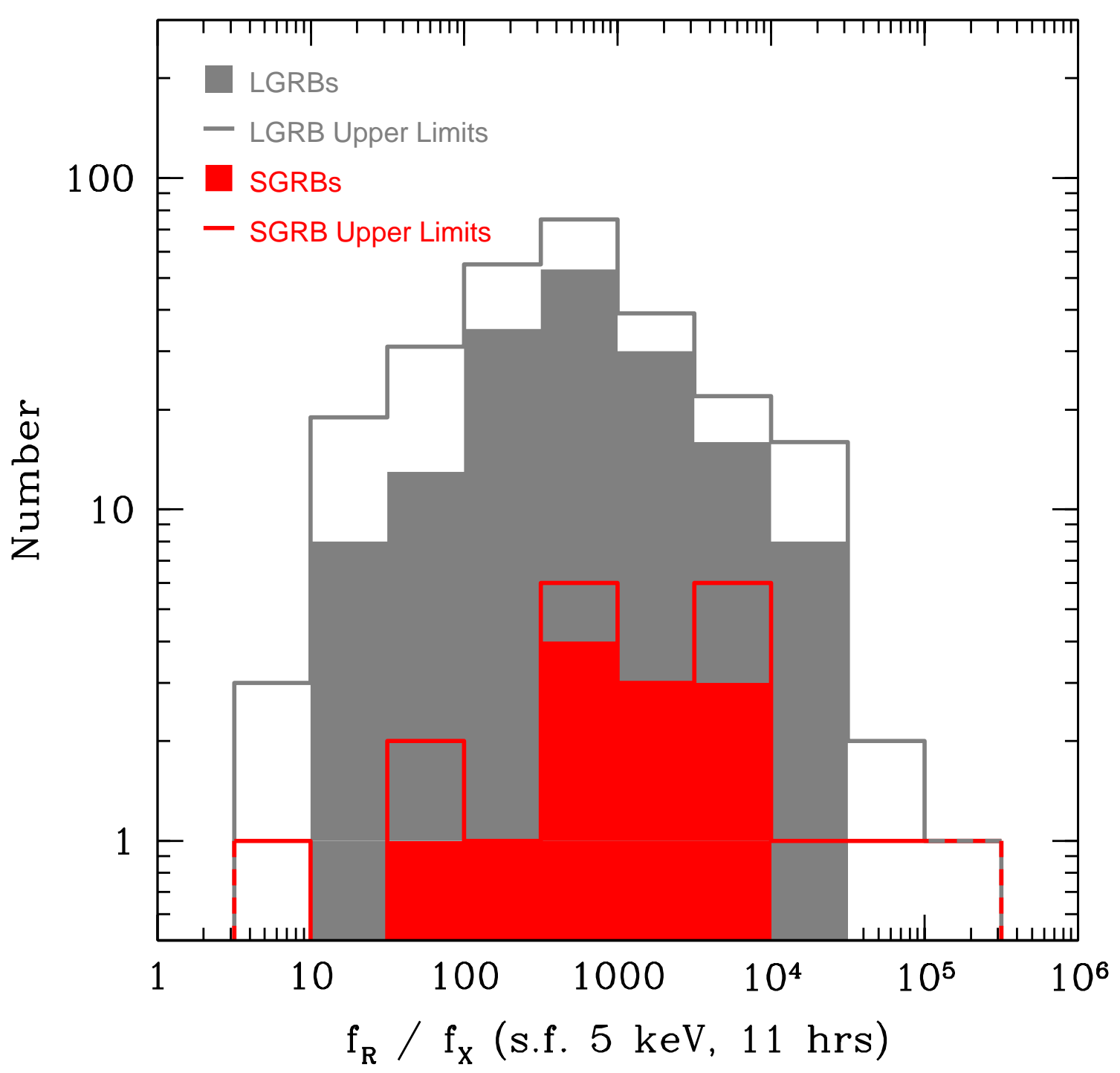

Fig. 8. - Histogram of the ratio of the observed fluxes at the optical and X-band after 11 hours (observed time). Long GRBs are marked in gray, and short ones in red. When only upper limits are known, empty boxes are drawn. Clearly, both samples show a nearly Gaussian shape in a log-normal plot, with similar means. 
Table 1:: Observed Prompt and Afterglow Properties of

Short-Duration GRBs

\begin{tabular}{|c|c|c|c|c|c|c|c|c|c|}
\hline GRB & Satellite & $\begin{array}{c}\text { Channel } \\
{[\mathrm{keV}]}\end{array}$ & $\mathrm{z}$ & $\begin{array}{c}\text { Fluence } \\
{\left[10^{-7}\right.} \\
\left.\mathrm{erg} \mathrm{cm}^{-2}\right]\end{array}$ & $\begin{array}{c}\log \\
\mathrm{E}_{\gamma, I S O} \\
{[\mathrm{erg}]}\end{array}$ & $\begin{array}{c}11 \mathrm{hr} \\
R \text {-band } \\
{[\mu \mathrm{Jy}]}\end{array}$ & 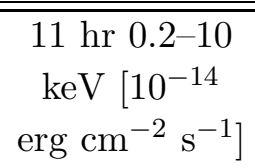 & $\begin{array}{l}\log \\
\mathrm{F}_{R} / \\
\mathrm{F}_{X}\end{array}$ & Ref. \\
\hline 001025B & Ulysses & $25-100$ & & 2.0 & & $<382.6$ & & & 1,2 \\
\hline 001204 & Ulysses & $25-100$ & & 8.6 & & $<190.9$ & & & 3,4 \\
\hline 010119 & Ulysses & $25-100$ & & 3.2 & & $<1970.3$ & & & 5,6 \\
\hline 010326B & HETE & $30-400$ & & $3.3 \pm 0.8$ & & $<26.0$ & & & 7,8 \\
\hline 020531 & HETE & $30-400$ & & $11.1_{-1.3}^{+1.4}$ & & $<16.9$ & & & 9,10 \\
\hline 020603 & Ulysses & $25-100$ & & 60.0 & & $<708.7$ & & & 11,12 \\
\hline 021201 & Ulysses & $25-100$ & & 2.0 & & $<58.0$ & & & 13,14 \\
\hline 050202 & Swift & $15-150$ & & $0.3 \pm 0.1$ & & $<55.3$ & & & 15,16 \\
\hline 050509B & Swift & $15-150$ & 0.225 & $0.09 \pm 0.02$ & $48.05_{-0.11}^{+0.09}$ & $<0.4$ & $2.9 \pm 1.0$ & $<2.93$ & $15,17,18$ \\
\hline 050709 & HETE & $30-400$ & 0.161 & $3.0 \pm 0.4$ & $49.06_{-0.18}^{+0.13}$ & $2.9_{-0.9}^{+1.2}$ & $2.8_{-1.4}^{+2.5}$ & $3.85_{-0.33}^{+0.31}$ & 19,20 \\
\hline 050724 & Swift & $15-150$ & 0.257 & $10.0 \pm 1.2$ & $50.21_{-0.06}^{+0.05}$ & $29.1 \pm 1.1$ & $20.5 \pm 6.6$ & $3.98_{-0.17}^{+0.12}$ & $15,21,22,23$ \\
\hline 050813 & Swift & $15-150$ & $0.722^{\dagger}$ & $0.4 \pm 0.1$ & $49.77_{-0.12}^{+0.10}$ & $<2.6$ & $0.21_{-0.18}^{+1.12}$ & $<4.92$ & $15,24,25,26$ \\
\hline 050906 & Swift & $15-150$ & & $0.06 \pm 0.02$ & & $<0.1$ & $<2.44^{a}$ & & $15,27,28$ \\
\hline 051105A & Swift & $15-150$ & & $0.22 \pm 0.04$ & & $<9.0$ & & & $15,29,30$ \\
\hline 051210 & Swift & $15-150$ & $0.114^{C}$ & $0.9 \pm 0.1$ & $48.36_{-0.08}^{+0.07}$ & $<2.1$ & $1.72_{-1.41}^{+6.66}$ & $<3.91$ & $15,24,31,32$ \\
\hline 051211 & HETE & $30-400$ & & $7.2 \pm 1.2$ & & $<6.4$ & & & $7,33,34$ \\
\hline 051221A & Swift & $15-150$ & 0.546 & $11.5 \pm 0.4$ & $50.95 \pm 0.01$ & $7.5_{-1.6}^{+2.1}$ & $99.4 \pm 24.5$ & $2.71_{-0.16}^{+0.14}$ & $15,21,35$ \\
\hline 051227 & Swift & $15-150$ & & $7.0 \pm 1.1$ & & $0.5 \pm 0.1$ & $9.3 \pm 2.5$ & & $15,21,36$ \\
\hline 060121 & HETE & $30-400$ & & $38.7 \pm 2.7$ & & $1.6_{-0.3}^{+0.4}$ & $157.0 \pm 26.4$ & & $7,21,37$ \\
\hline 060313 & Swift & $15-150$ & & $11.3 \pm 0.5$ & & $9.9_{-3.6}^{+5.5}$ & $65.5 \pm 14.6$ & & $15,21,36$ \\
\hline 060502B & Swift & $15-150$ & $0.287^{\dagger}$ & $0.4 \pm 0.1$ & $48.90_{-0.06}^{+0.05}$ & $<1.0$ & $1.2_{-0.5}^{+0.6}$ & $<3.73$ & $15,24,38,39$ \\
\hline 060801 & Swift & $15-150$ & $1.131^{\dagger}$ & $0.8 \pm 0.1$ & $50.43_{-0.06}^{+0.05}$ & $<0.5$ & $0.6_{-0.5}^{+2.0}$ & $<3.75$ & $15,24,40,41$ \\
\hline 061006 & Swift & $15-150$ & 0.438 & $14.2 \pm 1.4$ & $50.84_{-0.05}^{+0.04}$ & $6.0 \pm 0.4$ & $34.4 \pm 8.9$ & $3.07_{-0.13}^{+0.10}$ & $15,21,36,42$ \\
\hline 061201 & Swift & $15-150$ & $0.084^{C}$ & $3.3 \pm 0.3$ & $48.68_{-0.04}^{+0.03}$ & $2.0 \pm 0.2$ & $24.9 \pm 7.7$ & $2.73_{-0.17}^{+0.13}$ & $15,21,43,44$ \\
\hline
\end{tabular}

Continued on Next Page... 
Table 1 - Continued

\begin{tabular}{|c|c|c|c|c|c|c|c|c|c|}
\hline GRB & Satellite & $\begin{array}{c}\text { Channel } \\
{[\mathrm{keV}]}\end{array}$ & $\mathrm{z}$ & $\begin{array}{c}\text { Fluence } \\
{\left[10^{-7}\right.} \\
\left.\text { erg } \mathrm{cm}^{-2}\right]\end{array}$ & $\begin{array}{c}\log \\
\mathrm{E}_{\gamma, I S O} \\
{[\mathrm{erg}]}\end{array}$ & $\begin{array}{c}11 \mathrm{hr} \\
R \text {-band } \\
{[\mu \mathrm{Jy}]}\end{array}$ & 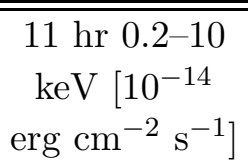 & $\begin{array}{l}\log \\
\mathrm{F}_{R} / \\
\mathrm{F}_{X}\end{array}$ & Ref. \\
\hline 061210 & Swift & $15-150$ & $0.410^{\dagger}$ & $11.1 \pm 1.8$ & $50.67_{-0.07}^{+0.06}$ & $<0.7$ & $580.8_{-3352}^{+581.3}$ & $<0.93$ & $15,24,36,45$ \\
\hline 061217 & Swift & $15-150$ & $0.827^{\dagger}$ & $0.4 \pm 0.1$ & $49.88_{-0.08}^{+0.07}$ & $<2.5$ & $0.2_{-0.1}^{+0.7}$ & $<5.00$ & $15,24,46,47$ \\
\hline 070209 & Swift & $15-150$ & & $0.2 \pm 0.1$ & & $<13.6$ & $<1.2$ & & $15,48,49$ \\
\hline 070406 & Swift & $15-150$ & & $0.4 \pm 0.1$ & & $<67.2$ & $<34.6$ & & $15,50,51$ \\
\hline 070429B & Swift & $15-150$ & 0.904 & $0.6 \pm 0.1$ & $50.13_{-0.08}^{+0.06}$ & $<0.3$ & $4.5_{-3.4}^{+10.7}$ & $<2.72$ & $15,24,52,53$ \\
\hline 070707 & Konus-Wind & $20-2000$ & & $14.1_{-10.7}^{+1.6}$ & & $2.4_{-0.6}^{+0.8}$ & $43.3 \pm 9.3$ & & $54,24,55$ \\
\hline 070714B & Swift & $15-150$ & 0.922 & $7.2 \pm 0.9$ & $51.21_{-0.06}^{+0.05}$ & $2.9_{-0.9}^{+1.1}$ & $13.6 \pm 3.0$ & $3.16_{-0.19}^{+0.17}$ & $56,21,57,58$ \\
\hline $070724 \mathrm{~A}$ & Swift & $15-150$ & $0.457^{\dagger}$ & $0.3 \pm 0.1$ & $49.20_{-0.12}^{+0.09}$ & $<0.2$ & $24.2 \pm 10.9$ & $<1.82$ & $59,21,60,61$ \\
\hline 070729 & Swift & $15-150$ & & $1.0 \pm 0.2$ & & $<5.4$ & $1.7 \pm 1.2$ & & $62,24,63$ \\
\hline 070809 & Swift & $15-150$ & & $1.0 \pm 0.1$ & & $0.9 \pm 0.2$ & $13.6 \pm 3.0$ & & $64,21,65$ \\
\hline 070810B & Swift & $15-150$ & & $0.12 \pm 0.03$ & & $<2.0$ & $<0.9^{a}$ & & $66,67,68$ \\
\hline 071112B & Swift & $15-150$ & & $0.5 \pm 0.1$ & & $<1.5$ & $<4.2$ & & 69,70 \\
\hline 071227 & Swift & $15-150$ & 0.384 & $2.3 \pm 0.3$ & $49.91 \pm 0.06$ & $2.2_{-0.7}^{+0.9}$ & $2.6 \pm 0.7$ & $3.77_{-0.21}^{+0.18}$ & $71,21,72,73$ \\
\hline
\end{tabular}

${ }^{\dagger}$ : probable redshift, ${ }^{C}$ : cluster redshift, ${ }^{a}$ Uses estimated $\mathrm{N}_{H}$, 1. Hurlev et al. (2000b), 2. Castro-Tirado et al. (2000), 3. Hurlev et al. (2000a), 4. Price et al. (2000), 5. Hurlev et al. (2001), 6. Price et al. (2001a), 7. Donaghv et al. (2006), 8. Price et al. (2001b), 9. Sakamoto et al. (2005), 10. Lamb et al. (2002), 11. Hurlev et al. (2002a), 12. Price et al. (2002), 13. Hurlev et al. (2002b), 14. Nysewander et al. (2002), 15. Sakamoto et al. (2008), 16. Castro-Tirado et al. (2005), 17. Gehrels et al. (2005), 18. Bloom et al. (2006), 19. Villasenor et al. (2005), 20. Fox et al. (2005), 21. Gehrels et al. (2008), 22. Malesani et al. (2007), 23. Berger et al. (2005), 24. Evans et al. (2007), 25. Bikmaev et al. (2005), 26. Berger (2005), 27. Levan et al. (2008), 28. Pagani et al. (2005), 29. Halpern et al. (2005a), 30. Mineo (2005), 31. Berger \& Boss (2005), 32. Berger \& Fox (2005), 33. Cusumano et al. (2005), 34. Guidorzi et al. (2005), 35. Soderberg et al. (2006a), 36. Berger et al. (2007a), 37. Levan et al. (2006b), 38. Price et al. (2006), 39. Bloom et al. (2007), 40. Piranomonte et al. (2006), 41. Cucchiara et al. (2006), 42. D' Avanzo et al. (2008), 43. Stratta et al. (2007b), 44. Berger (2007b), 45. Cenko et al. (2006), 46. Cobb (2006), 47. Berger (2006a), 48. Stratta et al. (2007c), 49. Johnson et al. (2007), 50. McBreen et al. (2007), 51. Zheng et al. (2007), 52. Holland et al. (2007), 53. Perlev et al. (2007), 54. Golenetskii et al. (2007), 55. Piranomonte et al. (2007), 56. Barbier et al. (2007), 57. Levan et al. (2007), 58. Graham et al. (2007) 
59. Ziaeepour et al. (2007a) 60. Cenko et al. (2007), 61. Cucchiara et al. (2007), 62. Guidorzi et al. (2007), 63. Berger \& Kaplan (2007), 64. Marshall et al. (2007a), 65. Perley et al. (2007a), 66. Marshall et al. (2007b), 67. Starling et al. (2007), 68. Xin et al. (2007), 69. Perri et al. (2007a), 70. Kocevski \& Bloom (2007), 71. Stratta et al. (2007a), 72. Wiersema et al. (2008), 73. Berger et al. (2007b) 
Table 2:: Observed Prompt and Afterglow Properties of

Long-Duration GRBs

\begin{tabular}{|c|c|c|c|c|c|c|c|c|c|}
\hline GRB & Satellite & $\begin{array}{c}\text { Channel } \\
{[\mathrm{keV}]}\end{array}$ & $\mathrm{z}$ & $\begin{array}{c}\text { Fluence } \\
{\left[10^{-7}\right.} \\
\left.\text { erg } \mathrm{cm}^{-2}\right]\end{array}$ & $\begin{array}{c}\log \\
\mathrm{E}_{I S O} \\
{[\mathrm{erg}]}\end{array}$ & $\begin{array}{c}11 \mathrm{hr} \\
R \text {-band } \\
{[\mu \mathrm{Jy}]}\end{array}$ & $\begin{array}{c}11 \mathrm{hr} 0.2-10 \\
\mathrm{keV}\left[10^{-14}\right. \\
\left.\mathrm{erg} \mathrm{cm} \mathrm{cm}^{-2} \mathrm{~s}^{-1}\right]\end{array}$ & $\begin{array}{l}\mathrm{F}_{R} / \\
\mathrm{F}_{X}\end{array}$ & Ref. \\
\hline 970111 & BeppoSAX & $40-700$ & & $430 \pm 30$ & & $<33.6$ & $7.5 \pm 4.7^{a}$ & & 1,2 \\
\hline 970228 & BeppoSAX & $40-700$ & 0.695 & 64.5 & $51.91_{-0.17}^{+0.12}$ & $22.0_{-3.5}^{+4.0}$ & $208.0 \pm 27.0^{a}$ & $2.86_{-0.15}^{+0.29}$ & $1,3,4$ \\
\hline 970402 & BeppoSAX & $40-700$ & & $82.0 \pm 9.0$ & & $<44.0$ & $13.5 \pm 7.3^{a}$ & & 1,5 \\
\hline 970508 & BeppoSAX & $40-700$ & 0.835 & 14.5 & $51.42_{-0.17}^{+0.12}$ & $6.5_{-1.3}^{+1.6}$ & $57.2 \pm 9.0^{a}$ & $2.97_{-0.21}^{+0.25}$ & $1,6,7$ \\
\hline 970616 & BATSE & $20-100$ & & $109.7 \pm 0.6$ & & $<14.0$ & $385.8_{-186.8}^{+208.3 b, d}$ & & $8,9,10$ \\
\hline 970815 & $B A T S E$ & $20-100$ & & $52.5 \pm 0.6$ & & $14.2_{-6.7}^{+12.3}$ & $498.9_{-286.4}^{+471.8 b}$ & & $8,11,12$ \\
\hline 970828 & BATSE & $20-100$ & 0.958 & 700.0 & $53.23_{-0.17}^{+0.12}$ & $<0.6$ & $198.8_{-91.1}^{+113.5 b, d}$ & $<1.26$ & $13,14,15$ \\
\hline 971214 & BeppoSAX & $40-700$ & 3.42 & 64.9 & $53.03_{-0.60}^{+0.24}$ & $5.6_{-0.4}^{+0.5}$ & $63.5 \pm 9.1^{a}$ & $2.97 \pm 0.29$ & 1,16 \\
\hline 971227 & BeppoSAX & $40-700$ & & $6.6 \pm 0.7$ & & $<5.8$ & $40.4_{-6.2}^{+14.3 a}$ & & $1,17,18$ \\
\hline 980326 & BeppoSAX & $40-700$ & & $7.5 \pm 1.5$ & & $11.9_{-1.1}^{+1.2}$ & $<152.6^{b, d}$ & & $1,19,20$ \\
\hline 980329 & BeppoSAX & $40-700$ & & $650 \pm 50$ & & $2.3_{-0.6}^{+0.7}$ & $59.9 \pm 5.6^{a}$ & & 1,21 \\
\hline 980425 & BeppoSAX & $40-700$ & 0.009 & $28.5 \pm 5.0$ & $47.47_{-0.51}^{+0.23}$ & $<24.6$ & $28.2 \pm 5.9^{a}$ & $<3.71$ & $1,22,23$ \\
\hline 980515 & BeppoSAX & $40-700$ & & $23.0 \pm 3.0$ & & & $56.0 \pm 22.0^{a}$ & & 1 \\
\hline 980519 & BeppoSAX & $40-700$ & & $81.0 \pm 5.0$ & & $62.4_{-9.0}^{+10.1}$ & $39.0_{-11.0}^{+12.0 a}$ & & 1,24 \\
\hline 980613 & BeppoSAX & $40-700$ & 1.097 & 9.9 & $51.31_{-0.60}^{+0.24}$ & $2.9_{-0.6}^{+0.7}$ & $26.0_{-11.0}^{+12.0 a}$ & $2.98_{-0.29}^{+0.32}$ & $1,25,26$ \\
\hline 980703 & BeppoSAX & $40-700$ & 0.966 & $300 \pm 100$ & $52.69_{-0.60}^{+0.24}$ & $35.5_{-9.2}^{+11.3}$ & $139.9_{-32.0}^{+69.9 a}$ & $3.33_{-0.17}^{+0.33}$ & $1,27,28$ \\
\hline
\end{tabular}

${ }^{a}: 1.6-10.0 \mathrm{keV},{ }^{b}: 2-10 \mathrm{keV},{ }^{c}: 0.5-6.0 \mathrm{keV},{ }^{d}:$ Uses estimated $\mathrm{N}_{H}, 1$. de Pasquale et al. (2006) 2. Castro-Tirado et al. (1997) 3. Galama et al. (1997), 4. Diorgovski et al. (1999), 5. Groot et al. (1997a), 6. Diorgovski et al. (1997), 7. Metzger et al. (1997), 8. Paciesas et al. (1999), 9. Connaughton et al. (1997), 10. Gorosabel et al. (1999), 11. Mirabal et al. (2005), 12. Soderberg et al. (2004a), 13. Groot et al. (1998a), 14. Murakami et al. (1997), 15. Diorgovski et al. (2001), 16. Diercks et al. (1998), 17. Antonelli et al. (1999), 18. Groot et al. (1997b), 19. Marshall et al. (1998), 20. Groot et al. (1998b), 21. Reichart et al. (1999), 22. Tinnev et al. (1998), 23. Galama et al. (1998a), 24. Vrba et al. (2000), 25. Diorgovski et al. (2003), 26. Hiorth et al. (2002), 27. Diorgovski et al. (1998), 28. Vreeswiik et al. (1999) 
Table 3: Best-fit Results of the Observed Properties of Long and Short GRBs

\begin{tabular}{lccccc}
\hline \hline & slope & zero-point & $\chi^{2} / d o f$ & zero-point, & $\chi^{2} / d o f$ \\
& $\alpha$ & $a$ & & $\mathrm{w} / \alpha=1$ & \\
Short, optical & $1.63 \pm 0.91$ & $10.66 \pm 7.52$ & 0.65 & $6.74 \pm 0.17$ & 0.73 \\
Long, optical & $0.97 \pm 0.11$ & $6.58 \pm 0.62$ & 1.30 & $6.78 \pm 0.05$ & 1.30 \\
Short, X-ray & $1.06 \pm 0.30$ & $-5.94 \pm 2.02$ & 0.67 & $-6.33 \pm 0.16$ & 0.66 \\
Long, X-ray & $1.10 \pm 0.09$ & $-5.59 \pm 0.54$ & 1.05 & $-6.18 \pm 0.04$ & 1.06 \\
\hline
\end{tabular}

Table 4: Best-fit Results of the Rest-Frame Properties of Long and Short GRBs

\begin{tabular}{lccccc}
\hline \hline & slope & zero-point & $\chi^{2} /$ dof & zero-point, & $\chi^{2} /$ dof \\
& $\alpha$ & $a$ & & w/ $\alpha=1$ & \\
Short, optical & $1.05 \pm 0.26$ & $-24.42 \pm 13.20$ & 0.31 & $-21.90 \pm 0.22$ & 0.31 \\
Long, optical & $0.93 \pm 0.09$ & $-19.20 \pm 4.44$ & 0.90 & $-21.68 \pm 0.07$ & 0.90 \\
Short, X-ray & $1.00 \pm 0.21$ & $-25.18 \pm 10.54$ & 0.71 & $-25.04 \pm 0.19$ & 0.71 \\
Long, X-ray & $1.05 \pm 0.07$ & $-27.32 \pm 3.73$ & 1.09 & $-24.74 \pm 0.07$ & 1.10 \\
\hline
\end{tabular}




\section{arXiv:0806.3607v2 [astro-ph] 27 May 2009:}

Table 1:: Observed Prompt and Afterglow Properties of

Short-Duration GRBs

\begin{tabular}{|c|c|c|c|c|c|c|c|c|c|c|}
\hline GRB & Satellite & $\begin{array}{c}\text { Channel } \\
{[\mathrm{keV}]}\end{array}$ & $\mathrm{z}$ & $\begin{array}{c}\text { Fluence } \\
{\left[10^{-7}\right.} \\
\left.\text { erg } \mathrm{cm}^{-2}\right]\end{array}$ & $\begin{array}{c}\log \\
\mathrm{E}_{\gamma, I S O} \\
{[\mathrm{erg}]}\end{array}$ & $\begin{array}{c}11 \mathrm{hr} \\
R \text {-band } \\
{[\mu \mathrm{Jy}]}\end{array}$ & $\begin{array}{c}11 \text { hr } 0.2-10 \\
\mathrm{keV}\left[10^{-14}\right. \\
\left.\text { erg } \mathrm{cm}^{-2} \mathrm{~s}^{-1}\right]\end{array}$ & $\begin{array}{c}\log \\
\mathrm{F}_{R} / \\
\mathrm{F}_{X}\end{array}$ & Ref. & \\
\hline 001025B & Ulysses & $25-100$ & & 2.0 & & $<382.6$ & & & 1,2 & \\
\hline 001204 & Ulysses & $25-100$ & & 8.6 & & $<190.9$ & & & 3,4 & \\
\hline 010119 & Ulysses & $25-100$ & & 3.2 & & $<1970.3$ & & & 5,6 & \\
\hline 010326B & HETE & $30-400$ & & $3.3 \pm 0.8$ & & $<26.0$ & & & 7,8 & \\
\hline 020531 & HETE & $30-400$ & & $11.1_{-1.3}^{+1.4}$ & & $<16.9$ & & & 9,10 & \\
\hline 020603 & Ulysses & $25-100$ & & 60.0 & & $<708.7$ & & & 11,12 & \\
\hline 021201 & Ulysses & $25-100$ & & 2.0 & & $<58.0$ & & & 13,14 & \\
\hline 050202 & Swift & $15-150$ & & $0.3 \pm 0.1$ & & $<55.3$ & & & 15,16 & \\
\hline 050509B & Swift & $15-150$ & 0.225 & $0.09 \pm 0.02$ & $48.05_{-0.11}^{+0.09}$ & $<0.4$ & $2.9 \pm 1.0$ & $<2.93$ & $15,17,18$ & \\
\hline 050709 & HETE & $30-400$ & 0.161 & $3.0 \pm 0.4$ & $49.06_{-0.18}^{+0.13}$ & $2.9_{-0.9}^{+1.2}$ & $2.8_{-1.4}^{+2.5}$ & $3.85_{-0.33}^{+0.31}$ & 19,20 & । \\
\hline 050724 & Swift & $15-150$ & 0.257 & $10.0 \pm 1.2$ & $50.21_{-0.06}^{+0.05}$ & $29.1 \pm 1.1$ & $20.5 \pm 6.6$ & $3.98_{-0.17}^{+0.12}$ & $15,21,22,23$ & । \\
\hline 050813 & Swift & $15-150$ & $0.722^{\dagger}$ & $0.4 \pm 0.1$ & $49.77_{-0.12}^{+0.10}$ & $<2.6$ & $0.21_{-0.18}^{+1.12}$ & $<4.92$ & $15,24,25,26$ & \\
\hline 050906 & Swift & $15-150$ & & $0.06 \pm 0.02$ & & $<0.1$ & $<2.44^{a}$ & & $15,27,28$ & \\
\hline $051105 \mathrm{~A}$ & Swift & $15-150$ & & $0.22 \pm 0.04$ & & $<9.0$ & & & $15,29,30$ & \\
\hline 051210 & Swift & $15-150$ & $0.114^{C}$ & $0.9 \pm 0.1$ & $48.36_{-0.08}^{+0.07}$ & $<2.1$ & $1.72_{-1.41}^{+6.66}$ & $<3.91$ & $15,24,31,32$ & \\
\hline 051211 & HETE & $30-400$ & & $7.2 \pm 1.2$ & & $<6.4$ & & & $7,33,34$ & \\
\hline 051221A & Swift & $15-150$ & 0.546 & $11.5 \pm 0.4$ & $50.95 \pm 0.01$ & $7.5_{-1.6}^{+2.1}$ & $99.4 \pm 24.5$ & $2.71_{-0.16}^{+0.14}$ & $15,21,35$ & \\
\hline 051227 & Swift & $15-150$ & & $7.0 \pm 1.1$ & & $0.5 \pm 0.1$ & $9.3 \pm 2.5$ & & $15,21,36$ & \\
\hline 060121 & HETE & $30-400$ & & $38.7 \pm 2.7$ & & $1.6_{-0.3}^{+0.4}$ & $157.0 \pm 26.4$ & & $7,21,37$ & \\
\hline 060313 & Swift & $15-150$ & & $11.3 \pm 0.5$ & & $9.9_{-3.6}^{+5.5}$ & $65.5 \pm 14.6$ & & $15,21,36$ & \\
\hline 060502B & Swift & $15-150$ & $0.287^{\dagger}$ & $0.4 \pm 0.1$ & $48.90_{-0.06}^{+0.05}$ & $<1.0$ & $1.2_{-0.5}^{+0.6}$ & $<3.73$ & $15,24,38,39$ & \\
\hline 060801 & Swift & $15-150$ & $1.131^{\dagger}$ & $0.8 \pm 0.1$ & $50.43_{-0.06}^{+0.05}$ & $<0.5$ & $0.6_{-0.5}^{+2.2}$ & $<3.75$ & $15,24,40,41$ & \\
\hline 061006 & Swift & $15-150$ & 0.438 & $14.2 \pm 1.4$ & $50.84_{-0.05}^{+0.04}$ & $6.0 \pm 0.4$ & $34.4 \pm 8.9$ & $3.07_{-0.13}^{+0.10}$ & $15,21,36,42$ & \\
\hline 061201 & Swift & $15-150$ & $0.084^{C}$ & $3.3 \pm 0.3$ & $48.68_{-0.04}^{+0.03}$ & $2.0 \pm 0.2$ & $24.9 \pm 7.7$ & $2.73_{-0.17}^{+0.13}$ & $15,21,43,44$ & \\
\hline
\end{tabular}

Continued on Next Page... 
Table 1 - Continued

\begin{tabular}{|c|c|c|c|c|c|c|c|c|c|}
\hline GRB & Satellite & $\begin{array}{c}\text { Channel } \\
{[\mathrm{keV}]}\end{array}$ & $\mathrm{z}$ & $\begin{array}{c}\text { Fluence } \\
{\left[10^{-7}\right.} \\
\left.\mathrm{erg} \mathrm{cm}^{-2}\right]\end{array}$ & $\begin{array}{c}\log \\
\mathrm{E}_{\gamma, I S O} \\
{[\mathrm{erg}]}\end{array}$ & $\begin{array}{c}11 \mathrm{hr} \\
R \text {-band } \\
{[\mu \mathrm{Jy}]}\end{array}$ & $\begin{array}{c}11 \mathrm{hr} 0.2-10 \\
\mathrm{keV}\left[10^{-14}\right. \\
\left.\mathrm{erg} \mathrm{cm}^{-2} \mathrm{~s}^{-1}\right]\end{array}$ & $\begin{array}{c}\log \\
\mathrm{F}_{R} / \\
\mathrm{F}_{X}\end{array}$ & Ref. \\
\hline 061210 & Swift & $15-150$ & $0.410^{\dagger}$ & $11.1 \pm 1.8$ & $50.67_{-0.07}^{+0.06}$ & $<0.7$ & $580.8_{-335.2}^{+581.3}$ & $<0.93$ & $15,24,36,45$ \\
\hline 061217 & Swift & $15-150$ & $0.827^{\dagger}$ & $0.4 \pm 0.1$ & $49.88_{-0.08}^{+0.07}$ & $<2.5$ & $0.2_{-0.1}^{+0.7}$ & $<5.00$ & $15,24,46,47$ \\
\hline 070209 & Swift & $15-150$ & & $0.2 \pm 0.1$ & & $<13.6$ & $<1.2$ & & $15,48,49$ \\
\hline 070406 & Swift & $15-150$ & & $0.4 \pm 0.1$ & & $<67.2$ & $<34.6$ & & $15,50,51$ \\
\hline 070429B & Swift & $15-150$ & 0.904 & $0.6 \pm 0.1$ & $50.13_{-0.08}^{+0.06}$ & $<0.3$ & $4.5_{-3.4}^{+10.7}$ & $<2.72$ & $15,24,52,53$ \\
\hline 070707 & Konus-Wind & $20-2000$ & & $14.1_{-10.7}^{+1.6}$ & & $2.4_{-0.6}^{+0.8}$ & $43.3 \pm 9.3$ & & $54,24,55$ \\
\hline 070714B & Swift & $15-150$ & 0.922 & $7.2 \pm 0.9$ & $51.21_{-0.06}^{+0.05}$ & $2.9_{-0.9}^{+1.1}$ & $13.6 \pm 3.0$ & $3.16_{-0.19}^{+0.17}$ & $56,21,57,58$ \\
\hline 070724A & Swift & $15-150$ & $0.457^{\dagger}$ & $0.3 \pm 0.1$ & $49.20_{-0.12}^{+0.09}$ & $<0.2$ & $24.2 \pm 10.9$ & $<1.82$ & $59,21,60,61$ \\
\hline 070729 & Swift & $15-150$ & & $1.0 \pm 0.2$ & & $<5.4$ & $1.7 \pm 1.2$ & & $62,24,63$ \\
\hline 070809 & Swift & $15-150$ & & $1.0 \pm 0.1$ & & $0.9 \pm 0.2$ & $13.6 \pm 3.0$ & & $64,21,65$ \\
\hline 070810B & Swift & $15-150$ & & $0.12 \pm 0.03$ & & $<2.0$ & $<0.9^{a}$ & & $66,67,68$ \\
\hline 071112B & Swift & $15-150$ & & $0.5 \pm 0.1$ & & $<1.5$ & $<4.2$ & & 69,70 \\
\hline 071227 & Swift & $15-150$ & 0.384 & $2.3 \pm 0.3$ & $49.91 \pm 0.06$ & $2.2_{-0.7}^{+0.9}$ & $2.6 \pm 0.7$ & $3.77_{-0.21}^{+0.18}$ & $71,21,72,73$ \\
\hline
\end{tabular}

${ }^{\dagger}$ : probable redshift, ${ }^{C}$ : cluster redshift, ${ }^{a}$ Uses estimated $\mathrm{N}_{H}$, 1. Hurlev et al. (2000c), 2. Castro-Tirado et al. (2000), 3. Hurlev et al. (2000b), 4. Price et al. (2000d), 5. Hurlev et al. (2001a), 6. Price et al. (2001a), 7. Donaghv et al. (2006), 8. Price et al. (2001b), 9. Sakamoto et al. (2005b), 10. Lamb et al. (2002), 11. Hurlev et al. (2002a), 12. Price et al. (2002c), 13. Hurlev et al. (2002i), 14. Nvsewander et al. (2002a), 15. Sakamoto et al. (2008), 16. Castro-Tirado et al. (2005), 17. Gehrels et al. (2005), 18. Bloom et al. (2006), 19. Villasenor et al. (2005), 20. Fox et al. (2005), 21. Gehrels et al. (2009), 22. Malesani et al. (2007a), 23. Berger et al. (2005a), 24. Evans et al. (2007), 25. Bikmaev et al. (2005), 26. Berger (2005), 27. Levan et al. (2008), 28. Pagani et al. (2005), 29. Halpern et al. (2005a), 30. Mineo (2005), 31. Berger \& Boss (2005), 32. Berger \& Fox (2005), 33. Cusumano et al. (2005), 34. Guidorzi et al. (2005a), 35. Soderberg et al. (2006a), 36. Berger et al. (2007c), 37. Levan et al. (2006b), 38. Price et al. (2006), 39. Bloom et al. (2007a), 40. Piranomonte et al. (2006a), 41. Cucchiara et al. (2006a), 42. D' Avanzo et al. (2008), 43. Stratta et al. (2007b), 44. Berger (2007), 45. Cenko et al. (2006a), 46. Cobb (2006), 47. Berger (2006a), 48. Stratta et al. (2007d), 49. Johnson et al. (2007), 50. McBreen et al. (2007a), 51. Zheng et al. (2007), 52. Holland et al. (2007a), 53. Perley et al. (2007c), 54. Golenetskii et al. (2007b), 55. Piranomonte et al. (2007), 56. Barbier et al. (2007), 57. Levan et al. (2007a), 58. Graham et al. (2007) 
59. Ziaeepour et al. (2007a), 60. Cenko et al. (2007a), 61. Cucchiara et al. (2007a), 62. Guidorzi et al. (2007a), 63. Berger \& Kaplan (2007), 64. Marshall et al. (2007a), 65. Perley et al. (2007a), 66. Marshall et al. (2007b), 67. Starling et al. (2007), 68. Xin et al. (2007a), 69. Perri et al. (2007a), 70. Kocevski \& Bloom (2007), 71. Stratta et al. (2007a), 72. Wiersema et al. (2008), 73. Berger et al. (2007b) 
Table 2:: Observed Prompt and Afterglow Properties of

Long-Duration GRBs (con’t)

\begin{tabular}{|c|c|c|c|c|c|c|c|c|c|}
\hline GRB & Satellite & $\begin{array}{c}\text { Channel } \\
{[\mathrm{keV}]}\end{array}$ & $\mathrm{z}$ & $\begin{array}{c}\text { Fluence } \\
{\left[10^{-7}\right.} \\
\left.\text { erg } \mathrm{cm}^{-2}\right]\end{array}$ & $\begin{array}{c}\log \\
\mathrm{E}_{\gamma, I S O} \\
{[\mathrm{erg}]}\end{array}$ & $\begin{array}{c}11 \mathrm{hr} \\
R \text {-band } \\
{[\mu \mathrm{Jy}]}\end{array}$ & 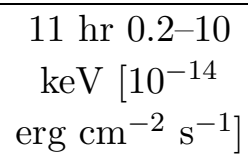 & $\begin{array}{l}\mathrm{Log} \\
\mathrm{F}_{R} / \\
\mathrm{F}_{X}\end{array}$ & Ref. \\
\hline 970111 & BeppoSAX & $40-700$ & & $430 \pm 30$ & & $<33.6$ & $7.5 \pm 4.7^{a}$ & & 1,2 \\
\hline 970402 & BeppoSAX & $40-700$ & & $82.0 \pm 9.0$ & & $<44.0$ & $13.5 \pm 7.3^{a}$ & & 1,5 \\
\hline 970508 & BeppoSAX & $40-700$ & 0.835 & 14.5 & $51.42_{-0.17}^{+0.12}$ & $6.5_{-1.3}^{+1.6}$ & $57.2 \pm 9.0^{a}$ & $2.97_{-0.21}^{+0.25}$ & $1,6,7$ \\
\hline 970828 & $B A T S E$ & $20-100$ & 0.958 & 700.0 & $53.23_{-0.17}^{+0.12}$ & $<0.6$ & $198.8_{-91.1}^{+113.5 b, d}$ & $<1.26$ & $13,14,15$ \\
\hline 971214 & BeppoSAX & $40-700$ & 3.42 & 64.9 & $53.03_{-0.60}^{+0.24}$ & $5.6_{-0.4}^{+0.5}$ & $63.5 \pm 9.1^{a}$ & $2.97 \pm 0.29$ & 1,16 \\
\hline 971227 & BeppoSAX & $40-700$ & & $6.6 \pm 0.7$ & & $<5.8$ & $40.4_{-6.2}^{+14.3 a}$ & & $1,17,18$ \\
\hline 980326 & BeppoSAX & $40-700$ & & $7.5 \pm 1.5$ & & $11.9_{-1.1}^{+1.2}$ & $<152.6^{b, d}$ & & 1,19, \\
\hline 980329 & BeppoSAX & $40-700$ & & $650 \pm 50$ & & $2.3_{-0.6}^{+0.7}$ & $59.9 \pm 5.6^{a}$ & & $1,21^{\prime}$ \\
\hline 980703 & BeppoSAX & $40-700$ & 0.966 & $300 \pm 100$ & $52.69_{-0.60}^{+0.24}$ & $35.5_{-9.2}^{+11.3}$ & $139.9_{-32.0}^{+69.9 a}$ & $3.33_{-0.17}^{+0.33}$ & $1,27,28$ \\
\hline 981220 & BeppoSAX & $40-700$ & & $100 \pm 20$ & & $<9.6$ & & & 29,30 \\
\hline 981226 & BeppoSAX & $40-700$ & & $4.0 \pm 1.0$ & & $12.0_{-3.3}^{+4.4}$ & $28.1_{-13.1}^{+21.1 a}$ & & 1,31 \\
\hline 990123 & BeppoSAX & $40-700$ & 1.6 & 1790.0 & $53.90_{-0.60}^{+0.24}$ & $37.8_{-2.9}^{+3.1}$ & $541.5 \pm 17.1^{a}$ & $2.80_{-0.17}^{+0.28}$ & 1,32 \\
\hline 990217 & BeppoSAX & $40-700$ & & $12.7 \pm 1.5$ & & $<1.0$ & $<28.0^{a}$ & & 1,33 \\
\hline 990308 & BATSE & $20-100$ & & $21.2 \pm 0.5$ & & $65.8_{-22.7}^{+34.2}$ & & & 8,34 \\
\hline 990506 & BATSE & $20-100$ & 1.307 & $342.6 \pm 0.6$ & $53.40_{-0.16}^{+0.12}$ & $<1.6$ & & & $8,35,36$ \\
\hline 990510 & BeppoSAX & $40-700$ & 1.619 & 181.0 & $52.91_{-0.60}^{+0.24}$ & $181.9_{-8.2}^{+8.5}$ & $346.7 \pm 21.1^{a}$ & $3.68_{-0.21}^{+0.26}$ & $1,37,38$ \\
\hline 990520 & BATSE & $20-200$ & & $8.0 \pm 3.0$ & & $<5.8$ & & & 39,40 \\
\hline
\end{tabular}


Table 2 - Continued

\begin{tabular}{|c|c|c|c|c|c|c|c|c|c|}
\hline GRB & Satellite & $\begin{array}{c}\text { Channel } \\
{[\mathrm{keV}]}\end{array}$ & $\mathrm{z}$ & $\begin{array}{c}\text { Fluence } \\
{\left[10^{-7}\right.} \\
\left.\text { erg } \mathrm{cm}^{-2}\right]\end{array}$ & $\begin{array}{c}\log \\
\mathrm{E}_{\gamma, I S O} \\
{[\mathrm{erg}]}\end{array}$ & $\begin{array}{c}11 \mathrm{hr} \\
R \text {-band } \\
{[\mu \mathrm{Jy}]}\end{array}$ & 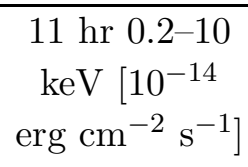 & $\begin{array}{l}\log \\
\mathrm{F}_{R} / \\
\mathrm{F}_{X}\end{array}$ & Ref. \\
\hline 990704 & BерроSAX & $40-700$ & & $10.0 \pm 1.0$ & & $<2.1$ & $58.9 \pm 8.4^{a}$ & & 1,43 \\
\hline 990705 & BeppoSAX & $40-700$ & 0.842 & 4230.0 & $53.72_{-0.60}^{+0.24}$ & $13.0_{-2.9}^{+3.5}$ & $<46.8^{a}$ & $>3.48$ & $1,44,45$ \\
\hline 990712 & BeppoSAX & $40-700$ & 0.434 & $65.0 \pm 3.0$ & $51.31_{-0.49}^{+0.22}$ & $26.2 \pm 1.0$ & & & $1,46,47$ \\
\hline 991105 & BATSE & $20-100$ & & $15.6 \pm 0.4$ & & $<4.6$ & & & 8,50 \\
\hline 991208 & Ulysses & $25-100$ & 0.707 & $490.0 \pm 49.0$ & $53.02_{-0.16}^{+0.12}$ & $392.5_{-164.9}^{+276.3}$ & & & $51,52,53$ \\
\hline 991216 & $B A T S E$ & 20-100 & 1.02 & $393.9 \pm 0.5$ & $53.25_{-0.16}^{+0.12}$ & $571.1_{-26.1}^{+27.3}$ & $780.7_{-238.1}^{+339.5 b}$ & $3.79_{-0.13}^{+0.26}$ & $8,54,55,56$ \\
\hline 000115 & $B A T S E$ & $20-100$ & & $82.9 \pm 0.2$ & & $<9.1$ & & & 8,57 \\
\hline 000126 & Ulysses & $25-100$ & & 100.0 & & $<11.4$ & & & 58,59 \\
\hline 000307 & Ulysses & $25-100$ & & 10.0 & & $<94.0$ & & & 67,68 \\
\hline 000323 & Ulysses & $25-100$ & & 43.0 & & $<235.1$ & & & 69,70 \\
\hline 000408 & Ulysses & $25-100$ & & 74.0 & & $<1467.3$ & & & $8,71,72$ \\
\hline 000416 & BерроSAX & $40-700$ & & $29000.0 \pm 0.3$ & & $<120.5$ & & & 73,74 \\
\hline 000418 & Ulysses & $25-100$ & 1.118 & 130.0 & $52.85_{-0.26}^{+0.16}$ & $32.1_{-14.3}^{+25.9}$ & & & $35,75,76$ \\
\hline 000424 & $B A T S E$ & $20-100$ & & $11.2 \pm 0.3$ & & $<11.6$ & & & 8,77 \\
\hline 000429 & $B A T S E$ & $20-100$ & & $51.3 \pm 0.5$ & & $<204.4$ & & & 8,78 \\
\hline 000508B & BATSE & $20-100$ & & $132.5 \pm 1.1$ & & $<39.8$ & & & 8,79 \\
\hline 000519 & BATSE & $20-100$ & & $19.4 \pm 0.2$ & & $<35.0$ & & & 8,80 \\
\hline 000528 & BeppoSAX & $40-700$ & & $14.4 \pm 0.4$ & & $<3.4$ & $30.0_{-14.0}^{+41.0 a}$ & & 1,81 \\
\hline
\end{tabular}


Table 2 - Continued

\begin{tabular}{|c|c|c|c|c|c|c|c|c|c|}
\hline GRB & Satellite & $\begin{array}{c}\text { Channel } \\
{[\mathrm{keV}]}\end{array}$ & $\mathrm{z}$ & $\begin{array}{c}\text { Fluence } \\
{\left[10^{-7}\right.} \\
\left.\text { erg } \mathrm{cm}^{-2}\right]\end{array}$ & $\begin{array}{c}\log \\
\mathrm{E}_{\gamma, I S O} \\
{[\mathrm{erg}]}\end{array}$ & $\begin{array}{c}11 \mathrm{hr} \\
R \text {-band } \\
{[\mu \mathrm{Jy}]}\end{array}$ & $\begin{array}{c}11 \mathrm{hr} 0.2-10 \\
\mathrm{keV}\left[10^{-14}\right. \\
\left.\mathrm{erg} \mathrm{cm} \mathrm{cm}^{-2} \mathrm{~s}^{-1}\right]\end{array}$ & $\begin{array}{l}\log \\
\mathrm{F}_{R} / \\
\mathrm{F}_{X}\end{array}$ & Ref. \\
\hline 000615 & BeppoSAX & $40-700$ & & $9.8 \pm 0.9$ & & $<19.1$ & $12.9 \pm 3.8^{a}$ & & 1,84 \\
\hline 000616 & Ulysses & $25-100$ & & 92.0 & & $<928.6$ & & & 85,86 \\
\hline 000623 & Ulysses & $25-100$ & & 50.0 & & $<138.4$ & & & 87,88 \\
\hline 000630 & Ulysses & $25-100$ & & 20.0 & & $3.3_{-0.7}^{+0.8}$ & & & 89,90 \\
\hline 000801 & Ulysses & $25-100$ & & 170.0 & & $<3.1$ & & & 91,92 \\
\hline 000812 & Ulysses & $25-100$ & & 200.0 & & $<30.2$ & & & 93,94 \\
\hline 000830 & Ulysses & $25-100$ & & 52.0 & & $<42.5$ & & & 95,96 \\
\hline 000911 & Ulysses & $25-100$ & 1.058 & 50.0 & $52.39_{-0.26}^{+0.16}$ & $57.3_{-19.3}^{+28.5}$ & & & 97,98 \\
\hline 000926 & Ulysses & $25-100$ & 2.038 & 220.0 & $53.57_{-0.26}^{+0.16}$ & $105.4_{-20.9}^{+26.1}$ & $325.7_{-86.9}^{+156.9 a}$ & $3.49_{-0.23}^{+0.34}$ & $1,99,100$ \\
\hline 001007 & Ulysses & $25-100$ & & 330.0 & & $139.5_{-75.5}^{+158.0}$ & & & 101,102 \\
\hline 001019 & Ulysses & $25-100$ & & 73.0 & & $<573.1$ & & & 103,104 \\
\hline 001025A & Ulysses & $25-100$ & & 32.0 & & $<0.7$ & $33.4_{-11.6}^{+17.6 b}$ & & 105,106 \\
\hline 001109 & BeppoSAX & $40-700$ & & $49.7 \pm 1.9$ & & $<2.5$ & $231.8_{-45.0}^{+58.0 a}$ & & 1,107 \\
\hline 001120 & Ulysses & $25-100$ & & 100.0 & & $<15.1$ & & & 108,109 \\
\hline 001212 & Ulysses & $25-100$ & & 120.0 & & $<143.7$ & & & 110,111 \\
\hline 010126 & HETE & $30-400$ & & 29.9 & & $<2.8$ & & & 112,113 \\
\hline 010213 & HETE & $30-400$ & & $0.7_{-0.3}^{+0.6}$ & & $<31.7$ & & & 114,115 \\
\hline 010214 & BeppoSAX & $40-700$ & & $45.0 \pm 0.8$ & & $4.0_{-1.1}^{+1.3}$ & $30.6_{-6.4}^{+7.1 a}$ & & 1,116 \\
\hline 010222 & BeppoSAX & $40-700$ & 1.477 & 753.0 & $53.45_{-0.60}^{+0.24}$ & $76.9_{-10.8}^{+12.0}$ & $705.4 \pm 34.0^{a}$ & $2.99_{-0.16}^{+0.29}$ & $1,117,118$ \\
\hline 010324 & Ulysses & $25-100$ & & 18.0 & & $<122.4$ & & & 119,120 \\
\hline 010326A & HETE & $30-400$ & & 160.0 & & $<31.7$ & & & 112,121 \\
\hline 010412 & Ulysses & $25-100$ & & 94.0 & & $<41.7$ & & & 122,123 \\
\hline 010629B & HETE & $30-400$ & & $28.6 \pm 2.7$ & & $38.4_{-10.1}^{+13.7}$ & & & 114,124 \\
\hline 010921 & HETE & $30-400$ & 0.45 & $113.0 \pm 9.0$ & $51.56_{-0.17}^{+0.12}$ & $143.7_{-31.6}^{+40.3}$ & & & 114,125 \\
\hline 011019 & HETE & $30-400$ & & $1.1 \pm 1.4$ & & $<145.7$ & & & 114,126 \\
\hline 011030 & BeppoSAX & $2-28$ & & 9.0 & & $<27.5$ & $1800 \pm 500^{b}$ & & $54,127,128$ \\
\hline
\end{tabular}


Table 2 - Continued

\begin{tabular}{|c|c|c|c|c|c|c|c|c|c|}
\hline GRB & Satellite & $\begin{array}{c}\text { Channel } \\
{[\mathrm{keV}]}\end{array}$ & $\mathrm{z}$ & $\begin{array}{c}\text { Fluence } \\
{\left[10^{-7}\right.} \\
\left.\text { erg } \mathrm{cm}^{-2}\right]\end{array}$ & $\begin{array}{c}\log \\
\mathrm{E}_{\gamma, I S O} \\
{[\mathrm{erg}]}\end{array}$ & $\begin{array}{c}11 \mathrm{hr} \\
R \text {-band } \\
{[\mu \mathrm{Jy}]}\end{array}$ & $\begin{array}{c}11 \mathrm{hr} 0.2-10 \\
\mathrm{keV}\left[10^{-14}\right. \\
\left.\mathrm{erg} \mathrm{cm}^{-2} \mathrm{~s}^{-1}\right]\end{array}$ & $\begin{array}{c}\log \\
\mathrm{F}_{R} / \\
\mathrm{F}_{X}\end{array}$ & Ref. \\
\hline 011130 & HETE & $30-400$ & & $1.0 \pm 0.6$ & & $61.5_{-30.5}^{+47.1}$ & & & $54,114,131$ \\
\hline 011211 & BeppoSAX & $40-700$ & 2.14 & $37.0 \pm 4.0$ & $52.44_{-0.49}^{+0.23}$ & $23.8 \pm 0.9$ & $17.2 \pm 0.5^{b}$ & $4.13_{-0.23}^{+0.27}$ & $1,54,132,133$ \\
\hline 011212 & HETE & $30-400$ & & $3.4 \pm 2.5$ & & $<58.2$ & & & 114,134 \\
\hline 020305 & HETE & $30-400$ & & 104.0 & & $67.6_{-28.0}^{+42.6}$ & & & 112,139 \\
\hline 020317 & HETE & $30-400$ & & $1.3 \pm 0.9$ & & $41.9_{-21.8}^{+37.3}$ & & & 114,140 \\
\hline 020321 & BeppoSAX & $40-700$ & & 30.0 & & $<38.5$ & $<34.0^{a}$ & & 1,141 \\
\hline 020331 & HETE & $30-400$ & & $53.0 \pm 9.0$ & & $16.2_{-1.8}^{+1.9}$ & & & 114,142 \\
\hline 020405 & Ulysses & $25-100$ & 0.695 & 300.0 & $52.79_{-0.26}^{+0.16}$ & $58.3_{-13.7}^{+17.8}$ & $710.0 \pm 30.0^{b}$ & $2.82_{-0.08}^{+0.29}$ & $54,143,144$ \\
\hline 020604 & Ulysses & $25-100$ & & 30.0 & & $<17.5$ & & & 152,153 \\
\hline 020714 & Ulysses & $25-100$ & & 83.0 & & $<5.1$ & & & 154,155 \\
\hline 020715 & Ulysses & $25-100$ & & 130.0 & & $<2.4$ & & & 156,157 \\
\hline 020801 & HETE & $30-400$ & & $95.0 \pm 11.0$ & & $<71.4$ & & & 114,158 \\
\hline 020812 & HETE & $30-400$ & & $19.0 \pm 8.0$ & & $<16.9$ & & & 114,159 \\
\hline 020813 & HETE & $30-400$ & 1.25 & $840.0 \pm 12.0$ & $53.34_{-0.17}^{+0.12}$ & $69.7_{-17.7}^{+23.7}$ & $393.0 \pm 8.0^{b}$ & $3.19_{-0.27}^{+0.22}$ & $54,114,160,161$ \\
\hline 020819B & HETE & $30-400$ & 0.41 & $63.0 \pm 8.0$ & $<4.7$ & $51.22_{-0.19}^{+0.13}$ & & & $114,162,163$ \\
\hline 020903 & HETE & $30-400$ & 0.25 & $0.2_{-0.1}^{+0.4}$ & $48.28_{-0.39}^{+0.48}$ & $<104.6$ & & & $114,164,165$ \\
\hline 021004 & HETE & $30-400$ & 2.3 & $18.0 \pm 7.0$ & $52.16_{-0.24}^{+0.18}$ & $139.3_{-5.2}^{+5.4}$ & $84.0 \pm 5.0^{b}$ & $4.21_{-0.23}^{+0.27}$ & $54,114,166,167$ \\
\hline 021008 & Ulysses & $25-100$ & & 850.0 & & $<713.1$ & & & 168,169 \\
\hline
\end{tabular}

Continued on Next Page... 
Table 2 - Continued

\begin{tabular}{|c|c|c|c|c|c|c|c|c|c|}
\hline GRB & Satellite & $\begin{array}{c}\text { Channel } \\
{[\mathrm{keV}]}\end{array}$ & $\mathrm{z}$ & $\begin{array}{c}\text { Fluence } \\
{\left[10^{-7}\right.} \\
\left.\text { erg } \mathrm{cm}^{-2}\right]\end{array}$ & $\begin{array}{c}\log \\
\mathrm{E}_{\gamma, I S O} \\
{[\mathrm{erg}]}\end{array}$ & $\begin{array}{c}11 \mathrm{hr} \\
R \text {-band } \\
{[\mu \mathrm{Jy}]}\end{array}$ & $\begin{array}{c}11 \mathrm{hr} 0.2-10 \\
\mathrm{keV}\left[10^{-14}\right. \\
\left.\mathrm{erg} \mathrm{cm}^{-2} \mathrm{~s}^{-1}\right]\end{array}$ & $\begin{array}{c}\log \\
\mathrm{F}_{R} / \\
\mathrm{F}_{X}\end{array}$ & Ref. \\
\hline 021104 & HETE & $30-400$ & & $6.0 \pm 4.0$ & & $<46.2$ & & & 114,173 \\
\hline 021112 & HETE & $30-400$ & & $2.1 \pm 1.1$ & & $<3.8$ & & & 114,174 \\
\hline 021113 & HETE & $8-40$ & & 5.0 & & $<4.2$ & & & 175,176 \\
\hline 030115A & HETE & $30-400$ & & $15.0 \pm 4.0$ & & $4.8_{-1.1}^{+1.4}$ & & & 114,181 \\
\hline 030131 & INTEGRAL & $20-200$ & & 70.0 & & $4.1_{-1.7}^{+2.4}$ & & & 182,183 \\
\hline 030204 & Ulysses & $25-100$ & & 220.0 & & $<11.5$ & & & 184,185 \\
\hline 030226 & HETE & $30-400$ & 1.98 & $43.0 \pm 7.0$ & $52.42_{-0.19}^{+0.13}$ & $47.8_{-5.8}^{+6.5}$ & $135.0 \pm 8.0^{b}$ & $3.53_{-0.19}^{+0.29}$ & $54,114,186$ \\
\hline 030227 & INTEGRAL & $20-200$ & & 7.5 & & $6.4_{-1.1}^{+1.3}$ & $89.6_{-30.7}^{+30.9 b}$ & & $179,187,\left.\right|_{\infty} 188$ \\
\hline 030329 & HETE & $30-400$ & 0.168 & $1076 \pm 13$ & $51.65_{-0.17}^{+0.12}$ & $4328.0 \pm 173.4$ & $5485.9_{-1158.3}^{+1477.4 b}$ & $3.75_{-0.13}^{+0.31}$ & $114,196,197,198$ \\
\hline 030413 & Ulysses & $25-100$ & & 23.0 & & $<148.7$ & & & 199,200 \\
\hline 030414 & Ulysses & $25-100$ & & 120.0 & & $<14086.9$ & & & 201,202 \\
\hline 030416 & HETE & $30-400$ & & $3.7 \pm 1.9$ & & $<38.8$ & & & 114,203 \\
\hline 030418 & HETE & $30-400$ & & $17.0 \pm 7.0$ & & $23.5_{-7.4}^{+9.6}$ & & & 114,204 \\
\hline 030429 & HETE & $30-400$ & 2.66 & $3.8 \pm 1.4$ & $51.59_{-0.26}^{+0.17}$ & $19.1_{-1.3}^{+1.4}$ & & & 114,205 \\
\hline 030528 & HETE & $30-400$ & 0.782 & $56.0 \pm 7.0$ & $51.75_{-0.18}^{+0.13}$ & $8.6_{-1.9}^{+2.2}$ & $29.1_{-18.4}^{+35.6 b}$ & $3.38_{-0.37}^{+0.38}$ & $114,206,207,208$ \\
\hline 030723 & HETE & $30-400$ & & $0.4_{-0.3}^{+0.6}$ & & $23.9_{-8.0}^{+10.6}$ & $14.5_{-5.9}^{+9.3 b}$ & & $114,209,210$ \\
\hline 030725 & HETE & $30-400$ & & $167.0 \pm 10.0$ & & $96.9_{-17.1}^{+20.7}$ & & & 114,211 \\
\hline 030823 & HETE & $30-400$ & & $13.0 \pm 4.0$ & & $<6.7$ & & & 114,212 \\
\hline
\end{tabular}

Continued on Next Page... 
Table 2 - Continued

\begin{tabular}{|c|c|c|c|c|c|c|c|c|c|}
\hline GRB & Satellite & $\begin{array}{c}\text { Channel } \\
{[\mathrm{keV}]}\end{array}$ & $\mathrm{z}$ & $\begin{array}{c}\text { Fluence } \\
{\left[10^{-7}\right.} \\
\left.\text { erg } \mathrm{cm}^{-2}\right]\end{array}$ & $\begin{array}{c}\mathrm{Log} \\
\mathrm{E}_{\gamma, I S O} \\
{[\mathrm{erg}]}\end{array}$ & $\begin{array}{c}11 \mathrm{hr} \\
R \text {-band } \\
{[\mu \mathrm{Jy}]}\end{array}$ & 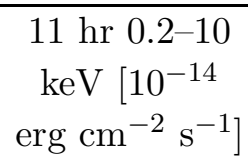 & $\begin{array}{l}\log \\
\mathrm{F}_{R} / \\
\mathrm{F}_{X}\end{array}$ & Ref. \\
\hline 031026 & HETE & $25-100$ & & 23.3 & & $<57.2$ & & & 215,216 \\
\hline 031111A & Ulysses & $25-100$ & & 21.0 & & $<865.3$ & & & 217,218 \\
\hline 031203 & INTEGRAL & 20-200 & 0.105 & 14.0 & $49.52_{-0.61}^{+0.17}$ & $191.5_{-25.0}^{+28.6}$ & $45.0 \pm 1.0^{b}$ & $4.47_{-0.08}^{+0.27}$ & $54,219,220,221$ \\
\hline 040223 & INTEGRAL & $20-200$ & & 20.0 & & $<2.1$ & $23.0 \pm 2.0^{b}$ & & 54,226 \\
\hline 040403 & INTEGRAL & $20-200$ & & 5.0 & & $<6.9$ & & & 227,228 \\
\hline 040422 & INTEGRAL & $20-200$ & & 3.4 & & $<2.7$ & & & 229 \\
\hline 040511 & HETE & $30-400$ & & 100.0 & & $<25.3$ & & & 230,231 \\
\hline 040624 & INTEGRAL & $20-200$ & & 10.0 & & $<2.9$ & & & 226,232 \\
\hline 040916 & HETE & $25-100$ & & $5.3 \pm 0.6$ & & $2.5 \pm 0.5$ & & & 242,243 \\
\hline 040924 & HETE & $30-400$ & 0.859 & 26.0 & $51.50_{-0.27}^{+0.16}$ & $6.9_{-1.1}^{+1.3}$ & & & $244,245,246$ \\
\hline 041006 & HETE & $30-400$ & 0.716 & 70.0 & $51.77_{-0.27}^{+0.16}$ & $18.1_{-1.9}^{+2.1}$ & $60.7_{-21.3}^{+21.7 c}$ & $3.38_{-0.18}^{+0.31}$ & $247,248,249$ \\
\hline 041016 & HETE & $25-100$ & & 46.9 & & $<121.3$ & & & 250,251 \\
\hline 041211 & HETE & $30-400$ & & 100.0 & & $<3.4$ & & & 252,253 \\
\hline 041217 & Swift & $15-150$ & & $27.7 \pm 1.2$ & & $<23.1$ & & & 254,255 \\
\hline 041219A & INTEGRAL & $15-350$ & & 1600.0 & & $142.1_{-61.6}^{+98.9}$ & & & 256,257 \\
\hline 041219C & Swift & $15-150$ & & $13.1 \pm 0.7$ & & $<141.5$ & & & 254,258 \\
\hline 041223 & Swift & $15-150$ & & $167.0 \pm 2.8$ & & $25.4_{-4.0}^{+4.5}$ & $273.7_{-61.3}^{+62.5 b}$ & & $254,259,260$ \\
\hline 041224 & Swift & $15-150$ & & $87.3 \pm 3.5$ & & $<81.0$ & & & 254,261 \\
\hline
\end{tabular}

Continued on Next Page... 
Table 2 - Continued

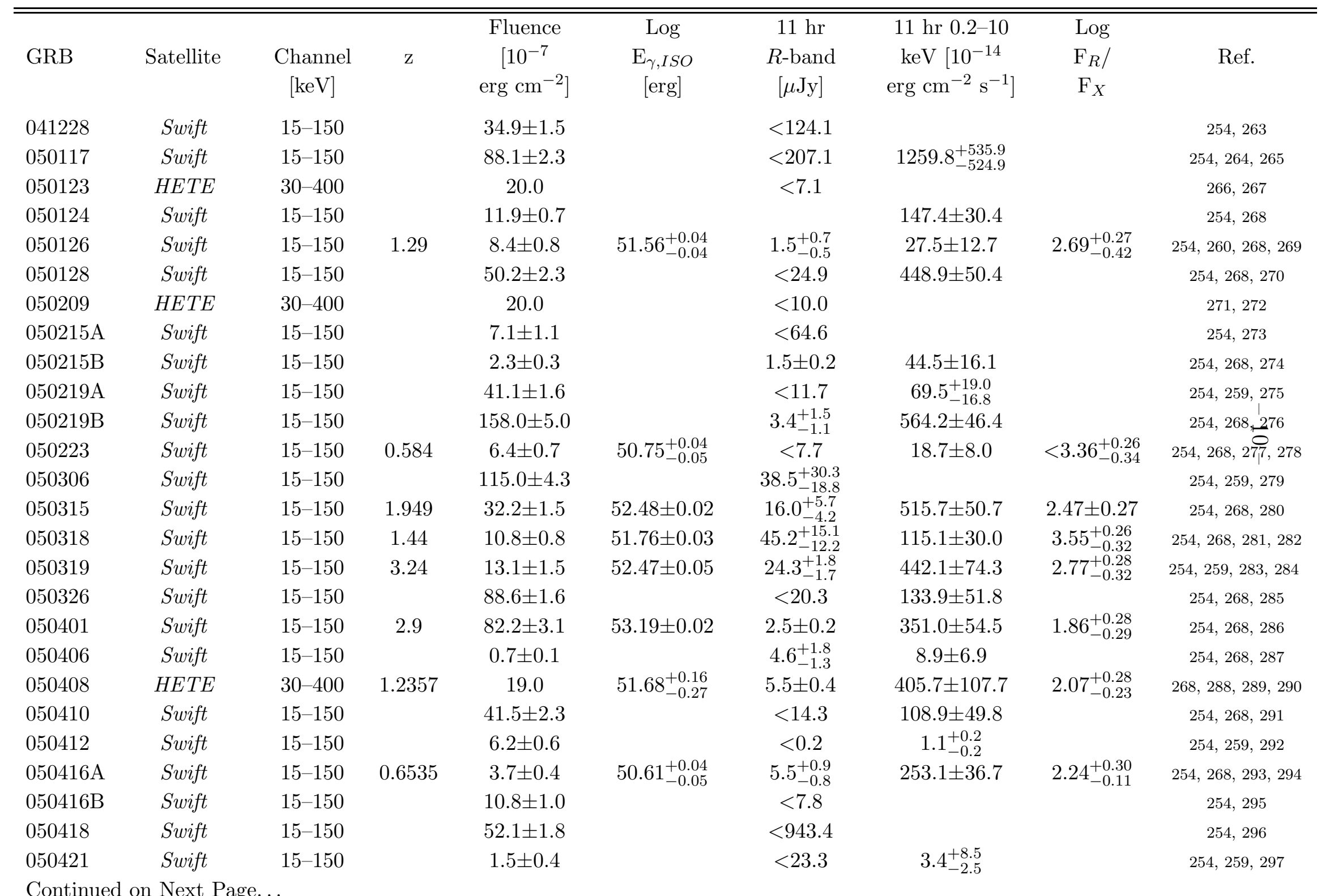


Table 2 - Continued

\begin{tabular}{|c|c|c|c|c|c|c|c|c|c|}
\hline GRB & Satellite & $\begin{array}{c}\text { Channel } \\
{[\mathrm{keV}]}\end{array}$ & $\mathrm{z}$ & $\begin{array}{c}\text { Fluence } \\
{\left[10^{-7}\right.} \\
\left.\text { erg } \mathrm{cm}^{-2}\right]\end{array}$ & $\begin{array}{c}\log \\
\mathrm{E}_{\gamma, I S O} \\
{[\mathrm{erg}]}\end{array}$ & $\begin{array}{c}11 \mathrm{hr} \\
R \text {-band } \\
{[\mu \mathrm{Jy}]}\end{array}$ & $\begin{array}{c}11 \mathrm{hr} 0.2-10 \\
\mathrm{keV}\left[10^{-14}\right. \\
\left.\mathrm{erg} \mathrm{cm}^{-2} \mathrm{~s}^{-1}\right]\end{array}$ & $\begin{array}{c}\log \\
\mathrm{F}_{R} / \\
\mathrm{F}_{X}\end{array}$ & Ref. \\
\hline 050502A & INTEGRAL & $20-200$ & 3.793 & 14.0 & $52.59_{-0.61}^{+0.24}$ & $3.6_{-0.7}^{+0.8}$ & $<5.8^{d}$ & $>3.87$ & $299,300,301,302$ \\
\hline 050504 & INTEGRAL & $20-200$ & & 15.0 & & $<9.0$ & $21.8 \pm 4.7$ & & $259,303,304$ \\
\hline 050505 & Swift & $15-150$ & 4.275 & $24.9 \pm 1.8$ & $52.95 \pm 0.03$ & $8.0_{-1.4}^{+1.7}$ & $757.3 \pm 90.6$ & $2.08_{-0.41}^{+0.27}$ & $254,268,305,306$ \\
\hline 050520 & INTEGRAL & $20-200$ & & 24.0 & & $<6.5$ & $22.7 \pm 10.5$ & & $268,311,312$ \\
\hline 050522 & INTEGRAL & $20-200$ & & 0.9 & & $<13.5$ & & & 313,314 \\
\hline $050525 \mathrm{~A}$ & Swift & $15-150$ & 0.606 & $153.0 \pm 2.2$ & $52.16 \pm 0.01$ & $151.9_{-33.9}^{+43.3}$ & $515.0 \pm 119.4$ & $3.36_{-0.20}^{+0.28}$ & $254,268,315,316$ \\
\hline 050528 & Swift & $15-150$ & & $4.5 \pm 0.7$ & & $<9.6$ & & & 254,317 \\
\hline 050603 & Swift & $15-150$ & 2.821 & $63.6 \pm 2.3$ & $53.06 \pm 0.02$ & $87.6_{-16.0}^{+19.3}$ & $330.1 \pm 44.4$ & $3.44_{-0.28}^{+0.29}$ & $254,268,318,319$ \\
\hline 050713A & Swift & $15-150$ & & $51.1 \pm 2.1$ & & $2.1_{-1.9}^{+11.8}$ & $465.4 \pm 65.1$ & & $254,268,326$ \\
\hline 050713B & Swift & $15-150$ & & $31.8 \pm 3.2$ & & $<24.9$ & $1180.0 \pm 236.8$ & & $254,268,327$ \\
\hline 050714A & INTEGRAL & $20-200$ & & 6.2 & & $<462.3$ & $265.7 \pm 72.2$ & & $259,328,329$ \\
\hline 050714B & Swift & $15-150$ & & $6.0 \pm 1.1$ & & $<3.3$ & $96.6 \pm 41.6$ & & $254,268,330$ \\
\hline 050716 & Swift & $15-150$ & & $61.7 \pm 2.4$ & & $2.5_{-0.7}^{+0.8}$ & $108.7 \pm 17.3$ & & $254,268,331$ \\
\hline 050717 & Swift & $15-150$ & & $63.1 \pm 1.8$ & & $<14.3$ & $25.5 \pm 5.9$ & & $254,268,332$ \\
\hline 050721 & Swift & $15-150$ & & $36.2 \pm 3.2$ & & $23.8_{-4.6}^{+5.7}$ & $202.8 \pm 37.4$ & & $254,268,333$ \\
\hline 050726 & Swift & $15-150$ & & $19.4 \pm 2.1$ & & $<9.7$ & $126.0 \pm 22.5$ & & $254,268,334$ \\
\hline 050730 & Swift & $15-150$ & 3.969 & $23.8 \pm 1.5$ & $52.88 \pm 0.03$ & $15.2_{-3.1}^{+3.7}$ & $766.9 \pm 41.1$ & $2.34_{-0.26}^{+0.31}$ & $254,268,335,336$ \\
\hline 050801 & Swift & $15-150$ & 1.56 & $3.1 \pm 0.5$ & $51.29_{-0.07}^{+0.06}$ & $18.4_{-7.7}^{+12.3}$ & $38.9 \pm 8.1$ & $3.63_{-0.41}^{+0.21}$ & $254,268,337,338$ \\
\hline 050802 & Swift & $15-150$ & 1.71 & $20.0 \pm 1.6$ & $52.17_{-0.04}^{+0.03}$ & $14.4_{-6.5}^{+9.6}$ & $323.8 \pm 38.6$ & $2.61_{-0.44}^{+0.22}$ & $254,268,339,340$ \\
\hline
\end{tabular}


Table 2 - Continued

\begin{tabular}{|c|c|c|c|c|c|c|c|c|c|}
\hline GRB & Satellite & $\begin{array}{c}\text { Channel } \\
{[\mathrm{keV}]}\end{array}$ & $\mathrm{z}$ & $\begin{array}{c}\text { Fluence } \\
{\left[10^{-7}\right.} \\
\left.\mathrm{erg} \mathrm{cm}^{-2}\right]\end{array}$ & $\begin{array}{c}\log \\
\mathrm{E}_{\gamma, I S O} \\
{[\mathrm{erg}]}\end{array}$ & $\begin{array}{c}11 \mathrm{hr} \\
R \text {-band } \\
{[\mu \mathrm{Jy}]}\end{array}$ & $\begin{array}{c}11 \text { hr } 0.2-10 \\
\mathrm{keV}\left[10^{-14}\right. \\
\left.\text { erg } \mathrm{cm}^{-2} \mathrm{~s}^{-1}\right]\end{array}$ & $\begin{array}{c}\log \\
\mathrm{F}_{R} / \\
\mathrm{F}_{X}\end{array}$ & Ref. \\
\hline 050803 & Swift & $15-150$ & & $21.5 \pm 1.4$ & & $<8.2$ & $464.1 \pm 74.1$ & & $254,268,341$ \\
\hline 050807 & HETE & $30-400$ & & 22.0 & & $<25.0$ & & & 342,343 \\
\hline 050814 & Swift & $15-150$ & 5.3 & $20.1 \pm 2.2$ & $53.00 \pm 0.05$ & $2.1_{-0.6}^{+0.8}$ & $138.3 \pm 30.6$ & $2.26_{-0.42}^{+0.32}$ & $254,268,344,345$ \\
\hline 050815 & Swift & $15-150$ & & $0.8 \pm 0.2$ & & & $8.7 \pm 3.0$ & & 254,268 \\
\hline 050819 & Swift & $15-150$ & & $3.5 \pm 0.6$ & & $<4.2$ & $62.1 \pm 31.1$ & & $254,268,346$ \\
\hline 050820A & Swift & $15-150$ & 2.612 & $34.4 \pm 2.4$ & $52.74 \pm 0.03$ & $103.2_{-24.9}^{+31.1}$ & $2212.0 \pm 134.8$ & $2.67_{-0.36}^{+0.25}$ & $254,268,347,348$ \\
\hline 050820B & Swift & $15-150$ & & $20.6 \pm 0.6$ & & & $15.4_{-6.8}^{+9.3}$ & & 254,259 \\
\hline 050822 & Swift & $15-150$ & & $24.6 \pm 1.7$ & & $<8.6$ & $352.5 \pm 45.2$ & & $254,268,349$ \\
\hline 050824 & Swift & $15-150$ & 0.83 & $2.7 \pm 0.5$ & $50.68_{-0.09}^{+0.08}$ & $19.9_{-1.4}^{+1.5}$ & $136.6 \pm 69.5$ & $3.08_{-0.35}^{+0.32}$ & $254,268,350,351$ \\
\hline 050826 & Swift & $15-150$ & 0.296 & $4.1 \pm 0.7$ & $49.95_{-0.08}^{+0.07}$ & $36.4_{-9.9}^{+12.7}$ & $145.2 \pm 78.9$ & $3.27_{-0.39}^{+0.30}$ & $254,268,352$ \\
\hline 050827 & Swift & $15-150$ & & $21.0 \pm 1.2$ & & $<6.7$ & $201.1_{-60.1}^{+62.2}$ & & $254,259,353$ \\
\hline 050904 & Swift & $15-150$ & 6.295 & $48.3 \pm 1.8$ & $53.49 \pm 0.20$ & $20.0_{-1.3}^{+1.4}$ & $5.0 \pm 0.2$ & $4.70_{-0.41}^{+0.31}$ & $254,268,354,355$ \\
\hline 050908 & Swift & $15-150$ & 3.344 & $4.8 \pm 0.5$ & $52.06_{-0.05}^{+0.04}$ & $8.1_{-1.9}^{+2.4}$ & $14.0 \pm 4.3$ & $3.79_{-0.35}^{+0.32}$ & $254,268,356,357$ \\
\hline 050915A & Swift & $15-150$ & & $8.5 \pm 0.9$ & & $1.0_{-0.7}^{+2.5}$ & $51.0 \pm 12.3$ & & $254,268,358$ \\
\hline 050915B & Swift & $15-150$ & & $33.8 \pm 1.4$ & & $5.4_{-2.0}^{+3.3}$ & $110.5 \pm 47.7$ & & $254,268,359$ \\
\hline 050916 & Swift & $15-150$ & & $9.3 \pm 1.3$ & & $<16739.7$ & $92.1 \pm 34.9$ & & $254,259,360$ \\
\hline 050922B & Swift & $15-150$ & & $22.3 \pm 3.6$ & & $<5.3$ & $199.5 \pm 102.3$ & & $254,268,361$ \\
\hline 050922C & Swift & $15-150$ & 2.198 & $16.2 \pm 0.5$ & $52.28_{-0.02}^{+0.01}$ & $23.9_{-5.5}^{+6.6}$ & $171.7 \pm 30.0$ & $3.13_{-0.33}^{+0.26}$ & $254,268,283,362$ \\
\hline 051001 & Swift & $15-150$ & & $17.4 \pm 1.5$ & & $<2.1$ & $26.6 \pm 2.9$ & & $254,268,363$ \\
\hline 051006 & Swift & $15-150$ & & $13.4 \pm 1.4$ & & $11.4_{-3.7}^{+5.1}$ & $6.2 \pm 0.9$ & & $254,259,364$ \\
\hline 051008 & Swift & $15-150$ & & $50.9 \pm 1.5$ & & $0.4_{-0.2}^{+0.6}$ & $206.0 \pm 34.5$ & & $254,268,365$ \\
\hline 051012 & Swift & $15-150$ & & $3.0 \pm 0.4$ & & $7.0_{-3.6}^{+3.8}$ & & & 254,366 \\
\hline 051016A & Swift & $15-150$ & & $8.4 \pm 1.4$ & & $<9.7$ & $30.9 \pm 9.8$ & & $254,268,367$ \\
\hline 051016B & Swift & $15-150$ & 0.936 & $1.7 \pm 0.2$ & $50.59_{-0.06}^{+0.05}$ & $4.9_{-1.5}^{+1.9}$ & $155.2 \pm 30.3$ & $2.42_{-0.28}^{+0.24}$ & $254,268,368,369$ \\
\hline 051021A & HETE & $30-400$ & & 60.0 & & $6.2_{-1.6}^{+2.2}$ & $137.1 \pm 29.2$ & & $268,370,371$ \\
\hline 051021B & Swift & $15-150$ & & $8.4 \pm 0.9$ & & $<813.1$ & $12.3 \pm 4.0$ & & $254,259,372$ \\
\hline
\end{tabular}


Table 2 - Continued

\begin{tabular}{|c|c|c|c|c|c|c|c|c|c|}
\hline GRB & Satellite & $\begin{array}{c}\text { Channel } \\
{[\mathrm{keV}]}\end{array}$ & $\mathrm{z}$ & $\begin{array}{c}\text { Fluence } \\
{\left[10^{-7}\right.} \\
\left.\text { erg } \mathrm{cm}^{-2}\right]\end{array}$ & $\begin{array}{c}\log \\
\mathrm{E}_{\gamma, I S O} \\
{[\mathrm{erg}]}\end{array}$ & $\begin{array}{c}11 \mathrm{hr} \\
R \text {-band } \\
{[\mu \mathrm{Jy}]}\end{array}$ & 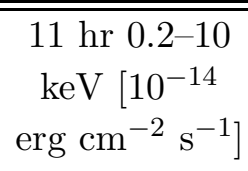 & $\begin{array}{l}\log \\
\mathrm{F}_{R} / \\
\mathrm{F}_{X}\end{array}$ & Ref. \\
\hline 051022 & HETE & $30-400$ & 0.8 & $1310.0 \pm 10.0$ & $53.14_{-0.17}^{+0.12}$ & $<0.4$ & $4265.0 \pm 350.9$ & $<-0.15_{-0.11}^{+0.28}$ & $268,373,374$ \\
\hline 051028 & HETE & $30-400$ & & 60.0 & & $6.3_{-0.7}^{+0.8}$ & $218.8 \pm 53.7$ & & $268,375,376$ \\
\hline 051109A & Swift & $15-150$ & 2.346 & $22.0 \pm 2.7$ & $52.46_{-0.06}^{+0.05}$ & $29.0_{-2.7}^{+2.9}$ & $997.0 \pm 76.1$ & $2.46_{-0.23}^{+0.28}$ & $254,268,377,378$ \\
\hline 051109B & Swift & $15-150$ & 0.08 & $2.6 \pm 0.4$ & $48.56_{-0.08}^{+0.07}$ & $3.1_{-2.7}^{+17.4}$ & $38.8 \pm 11.0$ & $2.75_{-0.85}^{+0.12}$ & $254,268,379,380$ \\
\hline 051117B & Swift & $15-150$ & & $1.4 \pm 0.4$ & & $<12.4$ & $2.4 \pm 0.9$ & & $254,259,384$ \\
\hline 051211B & INTEGRAL & $20-200$ & & 20.0 & & & $135.8 \pm 34.9$ & & 268,385 \\
\hline 051221B & Swift & $15-150$ & & $9.2 \pm 1.0$ & & $<21.4$ & $<11.2$ & & $254,386,387$ \\
\hline 060102 & Swift & $15-150$ & & $2.3 \pm 0.5$ & & $<18.2$ & $<152.2^{d}$ & & $254,388,389$ \\
\hline 060105 & Swift & $15-150$ & & $176.0 \pm 3.0$ & & $<44.1$ & $378.1 \pm 314.1$ & & $254,268,390$ \\
\hline 060111B & Swift & $15-150$ & & $16.0 \pm 1.4$ & & $3.9_{-3.1}^{+11.5}$ & $51.5 \pm 12.8$ & & $254,268,395$ \\
\hline 060114 & INTEGRAL & $20-200$ & & 13.0 & & $<4.5$ & $<38.4^{d}$ & & $396,397,398$ \\
\hline 060115 & Swift & $15-150$ & 3.53 & $17.1 \pm 1.5$ & $52.65 \pm 0.04$ & $5.2_{-3.9}^{+11.6}$ & $106.2 \pm 25$ & $2.73_{-0.92}^{+0.22}$ & $254,268,399,400$ \\
\hline 060116 & Swift & $15-150$ & & $24.1 \pm 2.6$ & & $6.9_{-3.9}^{+8.6}$ & $58.4 \pm 13.2$ & & $254,268,401$ \\
\hline 060117 & Swift & $15-150$ & & $202.0 \pm 3.7$ & & $714.2_{-596.0}^{+2593.9}$ & & & 254,402 \\
\hline 060123 & Swift & $15-150$ & & $3.0 \pm 1.2$ & & $<99.7$ & $275.8_{-74.7}^{+81.9}$ & & $259,403,404$ \\
\hline 060124 & Swift & $15-150$ & 2.296 & $4.6 \pm 0.5$ & $51.76 \pm 0.05$ & $92.8_{-19.6}^{+24.7}$ & $2873.0 \pm 370.7$ & $2.50_{-0.19}^{+0.31}$ & $254,268,405,406$ \\
\hline 060130 & INTEGRAL & $20-200$ & & 3.0 & & $<4.0$ & & & 407,408 \\
\hline 060202 & Swift & $15-150$ & 0.783 & $21.3 \pm 1.7$ & $51.53_{-0.04}^{+0.03}$ & $3.3_{-1.0}^{+1.3}$ & $719.2 \pm 90.1$ & $1.57_{-0.16}^{+0.26}$ & $254,268,409,410$ \\
\hline 060203 & Swift & $15-150$ & & $9.0 \pm 1.3$ & & $5.4_{-2.1}^{+3.0}$ & $72.6 \pm 18.8$ & & $254,268,411$ \\
\hline
\end{tabular}

Continued on Next Page... 
Table 2 - Continued

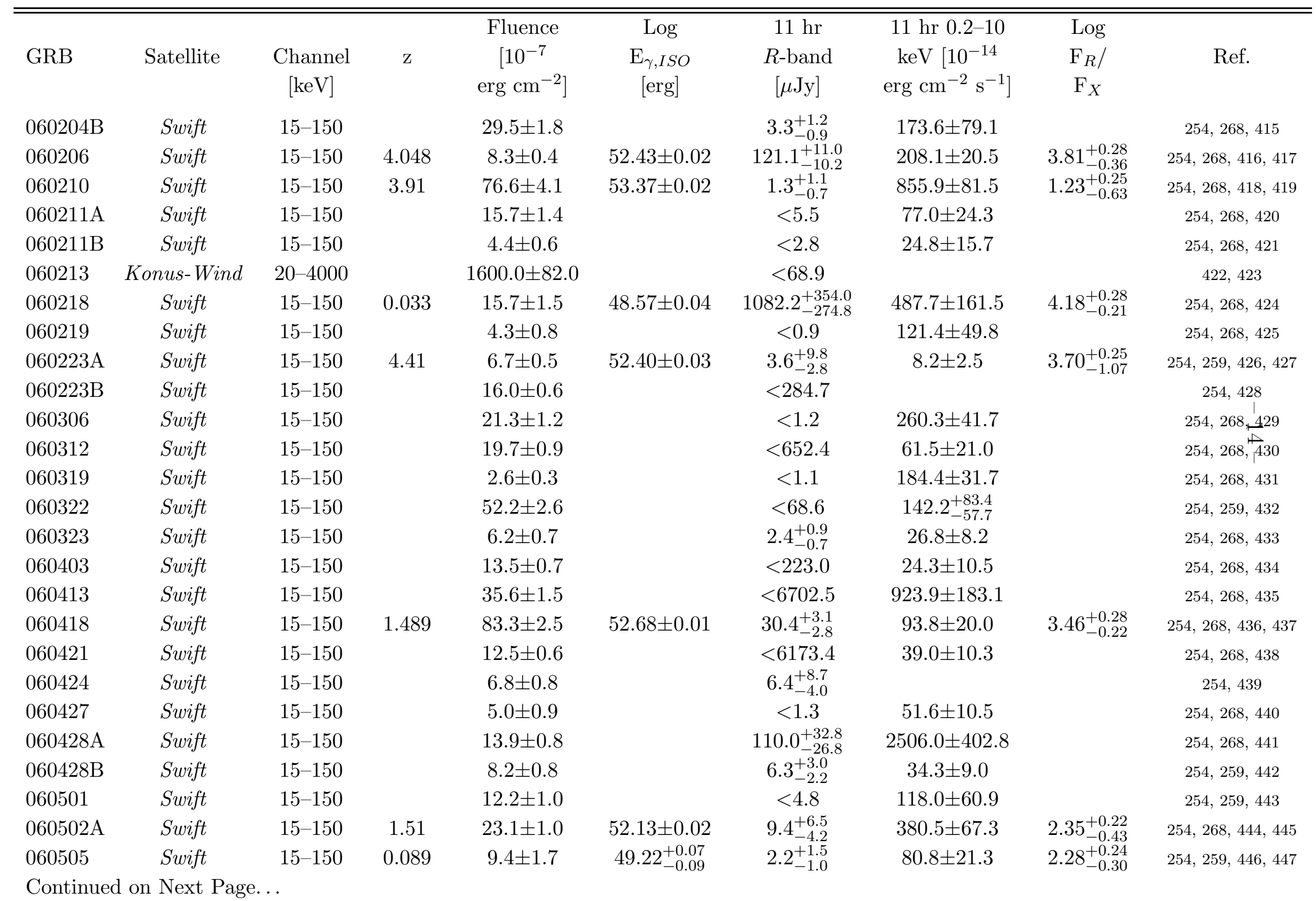


Table 2 - Continued

\begin{tabular}{|c|c|c|c|c|c|c|c|c|c|}
\hline GRB & Satellite & $\begin{array}{c}\text { Channel } \\
{[\mathrm{keV}]}\end{array}$ & $\mathrm{z}$ & $\begin{array}{c}\text { Fluence } \\
{\left[10^{-7}\right.} \\
\left.\text { erg } \mathrm{cm}^{-2}\right]\end{array}$ & $\begin{array}{c}\log \\
\mathrm{E}_{\gamma, I S O} \\
{[\mathrm{erg}]}\end{array}$ & $\begin{array}{c}11 \mathrm{hr} \\
R \text {-band } \\
{[\mu \mathrm{Jy}]}\end{array}$ & $\begin{array}{c}11 \mathrm{hr} 0.2-10 \\
\mathrm{keV}\left[10^{-14}\right. \\
\left.\mathrm{erg} \mathrm{cm}^{-2} \mathrm{~s}^{-1}\right]\end{array}$ & $\begin{array}{l}\log \\
\mathrm{F}_{R} / \\
\mathrm{F}_{X}\end{array}$ & Ref. \\
\hline 060510A & Swift & $15-150$ & & $80.5 \pm 3.1$ & & $20.0_{-14.6}^{+51.1}$ & $3309.0 \pm 639.1$ & & $254,268,449$ \\
\hline 060510B & Swift & $15-150$ & 4.9 & $40.7 \pm 1.8$ & $53.25 \pm 0.02$ & $0.4_{-0.3}^{+1.3}$ & $21.1 \pm 7.5$ & $2.31_{-1.35}^{+0.26}$ & $254,268,450,451$ \\
\hline 060512 & Swift & $15-150$ & 0.443 & $2.3 \pm 0.4$ & $50.06_{-0.08}^{+0.07}$ & $18.3_{-3.9}^{+4.6}$ & $22.8 \pm 6.7$ & $3.79_{-0.22}^{+0.27}$ & $254,268,452,453$ \\
\hline 060526 & Swift & $15-150$ & 3.221 & $12.6 \pm 1.7$ & $52.45_{-0.06}^{+0.05}$ & $45.9 \pm 1.5$ & $156.6 \pm 69.0$ & $3.49_{-0.42}^{+0.31}$ & $254,268,283,457$ \\
\hline 060602A & Swift & $15-150$ & & $16.1 \pm 1.6$ & & $0.2_{-0.1}^{+0.4}$ & $65.9_{-36.6}^{+58.7 d}$ & & $254,259,458$ \\
\hline 060604 & Swift & $15-150$ & 2.68 & $4.0 \pm 1.1$ & $51.82_{-0.13}^{+0.10}$ & $8.6_{-2.1}^{+2.7}$ & $163.8 \pm 31.6$ & $2.72_{-0.34}^{+0.28}$ & $254,268,459,460$ \\
\hline 060605 & Swift & $15-150$ & 3.711 & $7.0 \pm 0.9$ & $52.30_{-0.06}^{+0.05}$ & $24.8 \pm 0.6$ & $60.9 \pm 13.8$ & $3.65_{-0.35}^{+0.29}$ & $254,268,461,462$ \\
\hline 060607A & Swift & $15-150$ & 3.082 & $25.5 \pm 1.1$ & $52.73 \pm 0.02$ & $33.6_{-12.6}^{+18.9}$ & $254.8 \pm 29.7$ & $3.14_{-0.46}^{+0.24}$ & $254,268,463,464$ \\
\hline 060712 & Swift & $15-150$ & & $12.4 \pm 2.2$ & & $<17.5$ & $62.7 \pm 15.4$ & & $254,268,469$ \\
\hline 060714 & Swift & $15-150$ & 2.711 & $28.3 \pm 1.7$ & $52.68 \pm 0.03$ & $20.9_{-5.8}^{+7.7}$ & $141.0 \pm 24.5$ & $3.18_{-0.36}^{+0.27}$ & $254,268,283,470$ \\
\hline 060717 & Swift & $15-150$ & & $0.7 \pm 0.2$ & & $<1.1$ & $13.1_{-5.0}^{+5.8}$ & & $254,259,471$ \\
\hline 060719 & Swift & $15-150$ & & $15.0 \pm 0.9$ & & $<0.1$ & $214.4 \pm 42.4$ & & $254,268,472$ \\
\hline 060728 & Swift & $15-150$ & & $2.3 \pm 0.7$ & & $<13.9$ & $<4.5$ & & $254,473,474$ \\
\hline 060729 & Swift & $15-150$ & 0.54 & $26.1 \pm 2.1$ & $51.29_{-0.04}^{+0.03}$ & $253.3_{-68.7}^{+91.9}$ & $3306.0 \pm 546.5$ & $2.77_{-0.17}^{+0.26}$ & $254,268,475$ \\
\hline 060804 & Swift & $15-150$ & & $6.0 \pm 1.0$ & & $67.9_{-53.1}^{+236.3}$ & $214.7 \pm 48.7$ & & $254,268,476$ \\
\hline $060805 \mathrm{~A}$ & Swift & $15-150$ & & $0.7 \pm 0.2$ & & $<4.8$ & $14.2 \pm 4.8$ & & $254,268,477$ \\
\hline 060805B & RHESSI & $30-10000$ & & $1130.0 \pm 46.0$ & & $<119.9$ & $234.8_{-83.6}^{+109.2}$ & & $259,478,479,480$ \\
\hline 060807 & Swift & $15-150$ & & $8.5 \pm 1.1$ & & & $265.3 \pm 89.6$ & & 254,268 \\
\hline
\end{tabular}


Table 2 - Continued

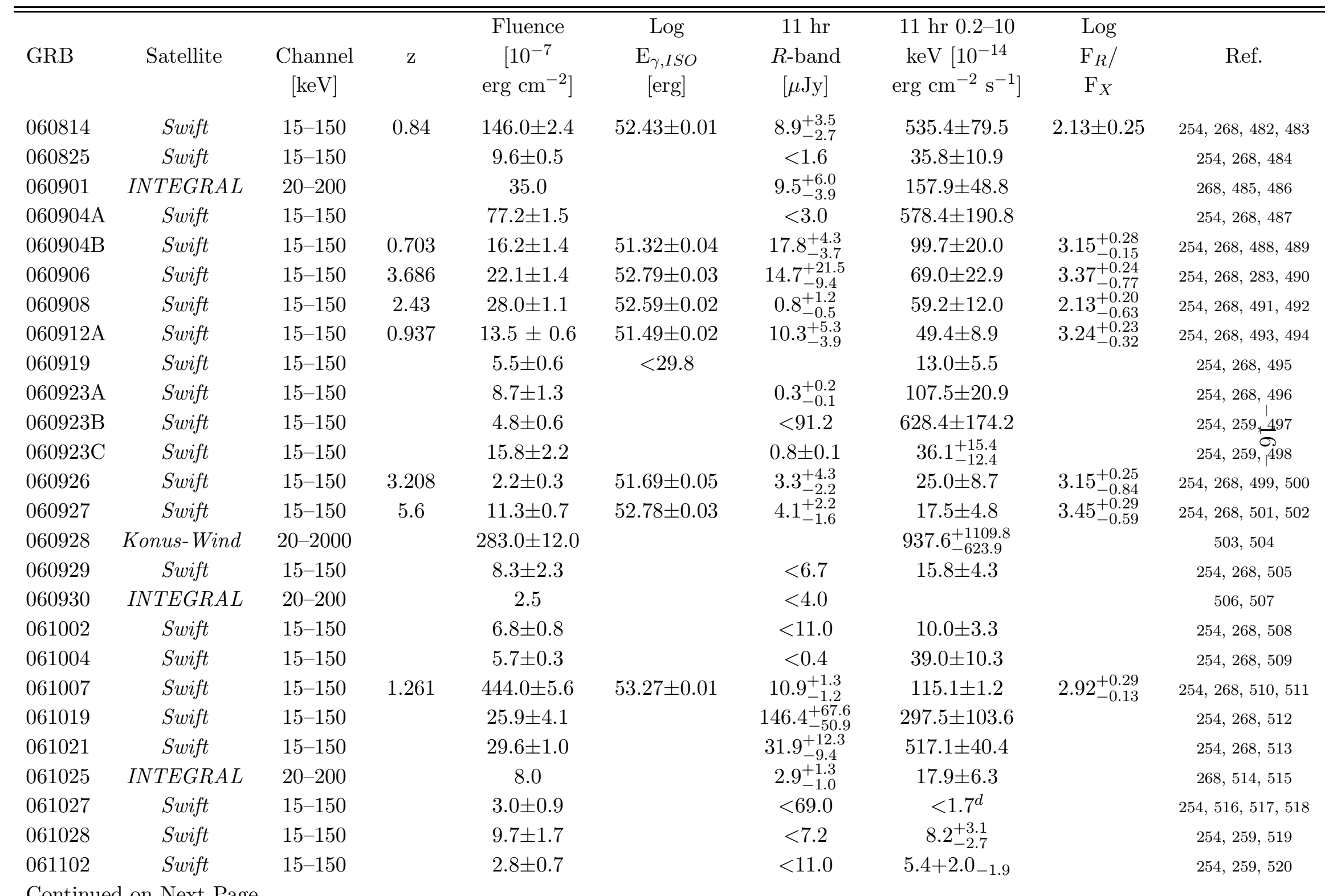


Table 2 - Continued

\begin{tabular}{|c|c|c|c|c|c|c|c|c|c|}
\hline GRB & Satellite & $\begin{array}{c}\text { Channel } \\
{[\mathrm{keV}]}\end{array}$ & $\mathrm{z}$ & $\begin{array}{c}\text { Fluence } \\
{\left[10^{-7}\right.} \\
\left.\text { erg } \mathrm{cm}^{-2}\right]\end{array}$ & $\begin{array}{c}\log \\
\mathrm{E}_{\gamma, I S O} \\
{[\mathrm{erg}]}\end{array}$ & $\begin{array}{c}11 \mathrm{hr} \\
R \text {-band } \\
{[\mu \mathrm{Jy}]}\end{array}$ & $\begin{array}{c}11 \mathrm{hr} 0.2-10 \\
\mathrm{keV}\left[10^{-14}\right. \\
\left.\mathrm{erg} \mathrm{cm}^{-2} \mathrm{~s}^{-1}\right]\end{array}$ & $\begin{array}{c}\log \\
\mathrm{F}_{R} / \\
\mathrm{F}_{X}\end{array}$ & Ref. \\
\hline 061110B & Swift & $15-150$ & 3.44 & $13.3 \pm 1.2$ & $52.52 \pm 0.04$ & $0.7_{-0.4}^{+0.9}$ & $12.2 \pm 3.9$ & $2.77_{-0.73}^{+0.24}$ & $254,259,523,524$ \\
\hline 061121 & Swift & $15-150$ & 1.314 & $137.0 \pm 2.0$ & $52.79 \pm 0.01$ & $23.2_{-4.6}^{+5.7}$ & $1151.0 \pm 114.8$ & $2.25_{-0.13}^{+0.31}$ & $254,268,525,526$ \\
\hline 061122 & INTEGRAL & $20-200$ & & 30.0 & & $6.4_{-1.0}^{+1.2}$ & $318.3 \pm 39.4$ & & $268,527,528$ \\
\hline 061218 & Swift & $15-150$ & & $0.4 \pm 0.2$ & & $<22.5$ & & & 254,532 \\
\hline 061222A & Swift & $15-150$ & & $79.9 \pm 1.6$ & & $0.3_{-0.1}^{+0.2}$ & $2821.0 \pm 307.4$ & & $254,268,533$ \\
\hline 061222B & Swift & $15-150$ & 3.355 & $22.4 \pm 1.8$ & $52.73_{-0.04}^{+0.03}$ & $1.2_{-0.9}^{+2.4}$ & $31.0 \pm 9.3$ & $2.62_{-0.88}^{+0.23}$ & $254,268,534,535$ \\
\hline 070103 & Swift & $15-150$ & & $3.4 \pm 0.5$ & & $<0.3$ & $33.0 \pm 7.6$ & & $254,268,536$ \\
\hline 070107 & Swift & $15-150$ & & $51.7 \pm 2.6$ & & $<42.4$ & $931.0 \pm 98.2$ & & $254,268,537$ \\
\hline 070208 & Swift & $15-150$ & 1.165 & $4.5 \pm 1.0$ & $51.20_{-0.11}^{+0.09}$ & $3.4_{-1.2}^{+1.6}$ & $63.7 \pm 20.1$ & $2.66_{-0.36}^{+0.24}$ & $254,268,545,546$ \\
\hline 070219 & Swift & $15-150$ & & $3.2 \pm 0.4$ & & $<0.1$ & $15.6 \pm 6.8$ & & $254,268,547$ \\
\hline 070220 & Swift & $15-150$ & & $104.0 \pm 2.3$ & & $<66.1$ & $105.9 \pm 25.7$ & & $254,268,548$ \\
\hline 070223 & Swift & $15-150$ & & $17.0 \pm 1.2$ & & $0.6_{-0.2}^{+0.3}$ & $68.9 \pm 20.7$ & & $254,268,549,550$ \\
\hline 070224 & Swift & $15-150$ & & $3.1 \pm 0.5$ & & $3.2_{-0.6}^{+0.7}$ & $60.3 \pm 38.9$ & & $254,268,551$ \\
\hline 070227 & Swift & $15-150$ & & $16.2 \pm 2.5$ & & $<49.7$ & $157.0_{-45.2}^{+45.9}$ & & $254,259,552$ \\
\hline 070306 & Swift & $15-150$ & 1.497 & $53.8 \pm 2.9$ & $52.49 \pm 0.02$ & $5.0_{-1.8}^{+2.7}$ & $161.2 \pm 398.0$ & $1.45_{-0.38}^{+0.22}$ & $254,259,553,554$ \\
\hline 070309 & INTEGRAL & $20-200$ & & 5.0 & & $<4007.4$ & $348.9 \pm 84.7$ & & $259,555,556$ \\
\hline 070311 & INTEGRAL & $20-200$ & & 20.0 & & $8.1_{-4.8}^{+11.4}$ & $135.3 \pm 27.6$ & & $259,557,558$ \\
\hline 070318 & Swift & $15-150$ & 0.836 & $24.8 \pm 1.1$ & $51.66 \pm 0.02$ & $34.0_{-5.1}^{+5.8}$ & $263.7 \pm 34.9$ & $3.02_{-0.11}^{+0.30}$ & $254,268,559,560$ \\
\hline
\end{tabular}

Continued on Next Page... 
Table 2 - Continued

\begin{tabular}{|c|c|c|c|c|c|c|c|c|c|}
\hline GRB & Satellite & $\begin{array}{c}\text { Channel } \\
{[\mathrm{keV}]}\end{array}$ & $\mathrm{z}$ & $\begin{array}{c}\text { Fluence } \\
{\left[10^{-7}\right.} \\
\left.\text { erg } \mathrm{cm}^{-2}\right]\end{array}$ & $\begin{array}{c}\log \\
\mathrm{E}_{\gamma, I S O} \\
{[\mathrm{erg}]}\end{array}$ & $\begin{array}{c}11 \mathrm{hr} \\
R \text {-band } \\
{[\mu \mathrm{Jy}]}\end{array}$ & 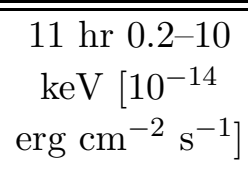 & $\begin{array}{l}\log \\
\mathrm{F}_{R} / \\
\mathrm{F}_{X}\end{array}$ & Ref. \\
\hline 070330 & Swift & $15-150$ & & $1.8 \pm 0.3$ & & $5.2_{-41}^{+16.7}$ & $52.2 \pm 20.5$ & & $254,268,562$ \\
\hline 070411 & Swift & $15-150$ & 2.954 & $27.0 \pm 1.6$ & $52.72_{-0.03}^{+0.02}$ & $13.9_{-5.2}^{+7.1}$ & $162.7 \pm 47.6$ & $2.95_{-0.48}^{+0.26}$ & $254,259,563,564$ \\
\hline 070412 & Swift & $15-150$ & & $4.8 \pm 0.7$ & & $<0.1$ & $65.4 \pm 19.0$ & & $254,268,565$ \\
\hline 070419A & Swift & $15-150$ & 0.97 & $5.6 \pm 0.8$ & $51.14_{-0.07}^{+0.06}$ & $2.1_{-0.7}^{+0.9}$ & $3.9 \pm 1.2$ & $3.66_{-0.31}^{+0.26}$ & $254,259,566,567$ \\
\hline 070429A & Swift & $15-150$ & & $9.1 \pm 1.4$ & & $<17.6$ & $110.7 \pm 27.1$ & & $254,268,570$ \\
\hline 070506 & Swift & $15-150$ & 2.31 & $2.1 \pm 0.2$ & $51.43 \pm 0.05$ & $8.1_{-40}^{+6.9}$ & $227.2 \pm 70.1$ & $2.54_{-0.32}^{+0.23}$ & $254,259,571,572$ \\
\hline 070508 & Swift & $15-150$ & 0.82 & $196.0 \pm 2.7$ & $52.54 \pm 0.01$ & $2.8_{-1.1}^{+1.6}$ & $447.6 \pm 25.3$ & $1.70_{-0.32}^{+0.22}$ & $254,268,573$ \\
\hline 070509 & Swift & $15-150$ & & $1.8 \pm 0.3$ & & $<3.5$ & $9.7 \pm 2.9$ & & $254,259,574$ \\
\hline 070517 & Swift & $15-150$ & & $2.2 \pm 0.4$ & & $<1.0$ & $43.9 \pm 12.0$ & & $254,268, \stackrel{\mid, 575}{-}$ \\
\hline 070529 & Swift & $15-150$ & 2.500 & $25.7 \pm 2.5$ & $52.58 \pm 0.04$ & $8.2_{-5.4}^{+15.5}$ & $69.7 \pm 14.7$ & $3.07_{-0.68}^{+0.20}$ & $254,268,580$ \\
\hline 070531 & Swift & $15-150$ & & $10.8 \pm 1.3$ & & $<7.9$ & $20.7_{-14.5}^{+43.0}$ & & $254,259,581$ \\
\hline 070611 & Swift & $15-150$ & 2.04 & $3.9 \pm 0.6$ & $51.60_{-0.07}^{+0.06}$ & $11.4_{-7.4}^{+20.3}$ & $29.3 \pm 6.1$ & $3.57_{-0.65}^{+0.19}$ & $254,259,582,583$ \\
\hline 070612A & Swift & $15-150$ & 0.617 & $106.0 \pm 6.0$ & $52.02_{-0.03}^{+0.02}$ & $329.8_{-128.6}^{+179.7}$ & & & $254,584,585$ \\
\hline 070612B & Swift & $15-150$ & & $16.8 \pm 1.2$ & & $<47.0$ & $10.8 \pm 5.9$ & & $254,268,586$ \\
\hline 070616 & Swift & $15-150$ & & $192.0 \pm 3.5$ & & $49.0_{-42.6}^{+240.0}$ & $377.7 \pm 70.4$ & & $254,268,587$ \\
\hline 070621 & Swift & $15-150$ & & $43.0 \pm 1.0$ & & $<1.0$ & $215.6 \pm 38.3$ & & $268,588,589$ \\
\hline 070628 & Swift & $15-150$ & & $35.0 \pm 2.0$ & & $139.4_{-83.0}^{+180.1}$ & $1728.0 \pm 150.1$ & & 268,590 \\
\hline 070704 & Swift & $15-150$ & & $59.0 \pm 3.0$ & & $<126.5$ & $91.1 \pm 21.1$ & & $268,591,592$ \\
\hline 070714A & Swift & $15-150$ & & $1.5 \pm 0.2$ & & $<3.2$ & $70.6 \pm 17.1$ & & $268,593,594$ \\
\hline 070721A & Swift & $15-150$ & & $0.7 \pm 0.2$ & & $0.7_{-0.6}^{+2.9}$ & $40.8 \pm 12.2$ & & 268,595 \\
\hline
\end{tabular}

Continued on Next Page. . 
Table 2 - Continued

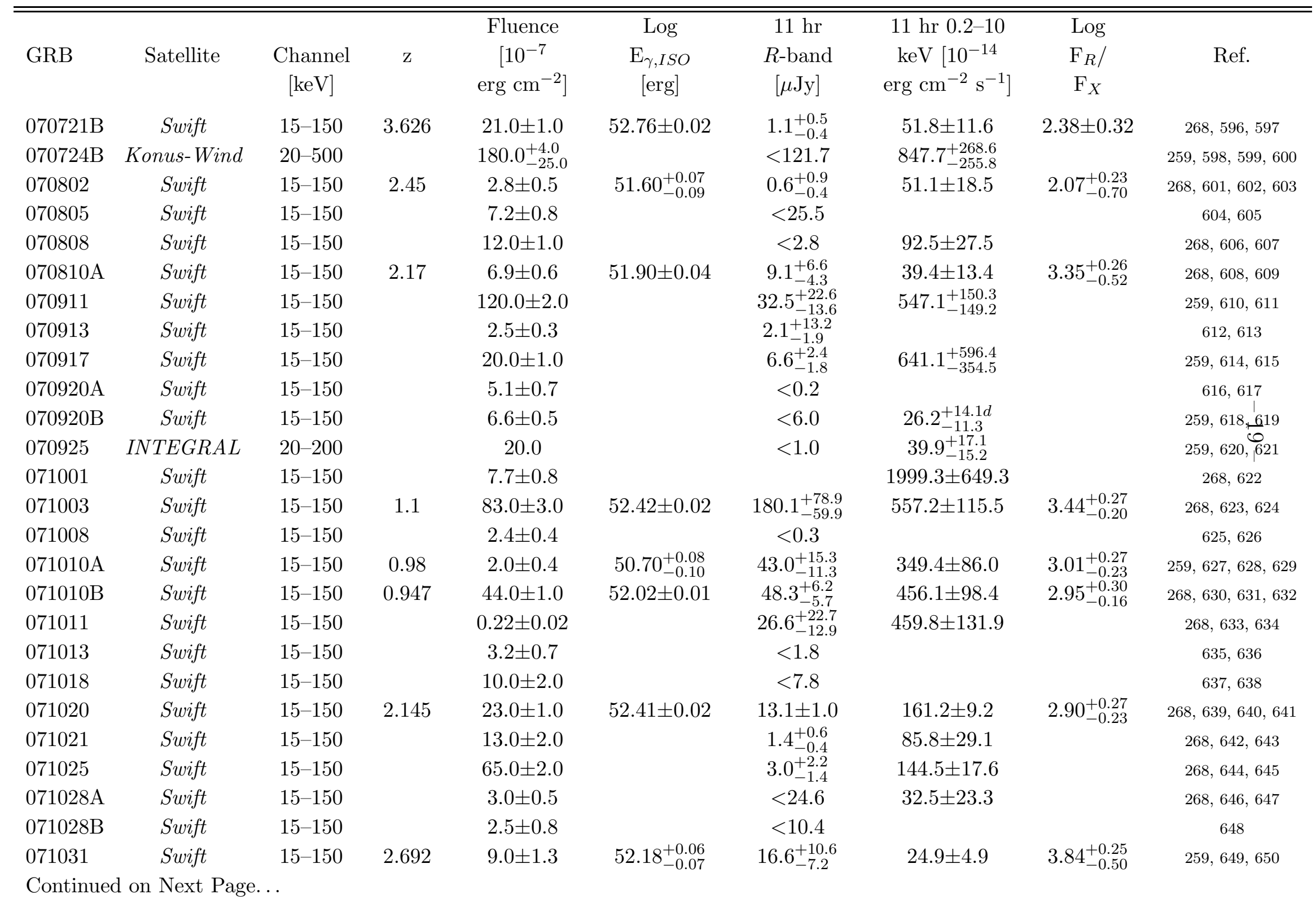


Table 2 - Continued

\begin{tabular}{|c|c|c|c|c|c|c|c|c|c|}
\hline GRB & Satellite & $\begin{array}{c}\text { Channel } \\
{[\mathrm{keV}]}\end{array}$ & z & $\begin{array}{c}\text { Fluence } \\
{\left[10^{-7}\right.} \\
\left.\text { erg } \mathrm{cm}^{-2}\right]\end{array}$ & $\begin{array}{c}\log \\
\mathrm{E}_{\gamma, I S O} \\
{[\mathrm{erg}]}\end{array}$ & $\begin{array}{c}11 \mathrm{hr} \\
R \text {-band } \\
{[\mu \mathrm{Jy}]}\end{array}$ & 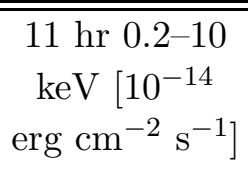 & $\begin{array}{l}\log \\
\mathrm{F}_{R} / \\
\mathrm{F}_{X}\end{array}$ & Ref. \\
\hline 071101 & Swift & $15-150$ & & $0.8 \pm 0.3$ & & $<415.3$ & $15.0_{-4.8}^{+4.8}$ & & $259,651,652$ \\
\hline 071109 & INTEGRAL & $20-200$ & & 6.6 & & $<140.7$ & 40.9 & & 653,654 \\
\hline $071112 \mathrm{C}$ & Swift & $15-150$ & 0.823 & $30.0 \pm 4.0$ & $51.73_{-0.06}^{+0.05}$ & $5.6_{-1.4}^{+1.7}$ & $37.0 \pm 1.6$ & $3.09_{-0.11}^{+0.27}$ & $268,655,656$ \\
\hline 071117 & Swift & $15-150$ & 1.331 & $24.0 \pm 1.0$ & $52.04 \pm 0.02$ & & $144.3 \pm 25.4$ & & $268,657,658$ \\
\hline
\end{tabular}

${ }^{a}: 1.6-10.0 \mathrm{keV},{ }^{b}: 2-10 \mathrm{keV},{ }^{c}: 0.5-6.0 \mathrm{keV},{ }^{d}$ : Uses estimated $\mathrm{N}_{H}, 1$. de Pasquale et al. (2006a) 2. Castro-Tirado et al. (1997) 3. Galama et al. (1997), 4. Diorgovski et al. (1999), 5. Groot et al. (1997a), 6. Diorgovski et al. (1997), 7. Metzger et al. (1997), 8. Paciesas et al. (1999), 9. Connaughton et al. (1997), 10. Gorosabel et al. (1999), 11. Mirabal et al. (2005), 12. Soderberg et al. (2004a), 13. Groot et al. (1998a), 14. Murakami et al. (1997), 15. Diorgovski et al. (2001), 16. Diercks et al. (1998), 17. Antonelli et al. (1999), 18. Groot et al. (1997bde 19. Marshall et al. (1998), 20. Groot et al. (1998b), 21. Reichart et al. (1999), 22. Tinney et al. (1998), 23. Galama et al. (1998), 24. Vrba et al. (2000), 25. Diorgovski et al. (2003), 26. Hiorth et al. (2002), 27. Diorgovski et al. (1998), 28. Vreeswiik et al. (1999a) 29. Frontera et al. (1998), 30. Pedersen et al. (1999a), 31. Castro-Tirado et al. (1998), 32. Kulkarni et al. (1999), 33. Palazzi et al. (1999a), 34. Schaefer et al. (1999), 35. Bloom et al. (2003), 36. Masetti et al. (1999), 37. Vreeswiik et al. (1999b), 38. Harrison et al. (1999), 39. Kippen et al. (1999), 40. Castro-Tirado et al. (1999), 41. Hurlev \& Cline (1999), 42. Pedersen et al. (1999b), 43. Jensen et al. (1999), 44. Le Floc'h et al. (2002), 45. Masetti et al. (2000a), 46. Galama et al. (1999), 47. Sahu et al. (2000), 48. Greiner et al. (1999), 49. Thorstensen et al. (1999), 50. Palazzi et al. (1999b), 51. Hurlev et al. (2000e), 52. Dodonov et al. (1999), 53. Castro-Tirado et al. (2001), 54. Gendre et al. (2006), 55. Vreeswiik et al. (1999c), 56. Halpern et al. (2000), 57. Gorosabel et al. (2000a), 58. Hurlev et al. (2000a), 59. Kiernsmo et al. (2000), 60. Hurlev et al. (2000d), 61. Andersen et al. (2000), 62. Piro et al. (2002), 63. Rhoads et al. (2000), 64. Jensen et al. (2001), 65. Castro et al. (2000), 66. Rhoads \& Fruchter (2001), 67. Hurley et al. (2000f), 68. Pedersen et al. (2000), 69. Hurley et al. (2000g), 70. Henden et al. (2000a), 71 . Hurley et al. (2000h), 72. Park et al. (2000), 73. Kippen \& Woods (2000), 74. Price et al. (2000a), 75. Hurlev et al. (2000i), 76. Klose et al. (2000), 77. Uglesich et al. (2000), 78. Henden (2000), 79. Jensen et al. (2000a), 80. Jensen et al. (2000b), 81. Palazzi et al. (2000a), 82. Hurlev et al. (2000i), 83. Diercks \& Cotoros (2000), 84. Stanek et al. (2000), 85. Hurlev et al. (2000k), 86. Bartolini et al. (2000), 87. Hurlev et al. (20001) 88. Gorosabel et al. (2000b), 89. Hurlev et al. (2000n), 90. Fvnbo et al. (2001a), 91. Hurlev et al. (2000m), 92. Palazzi et al. (2000b), 93. Hurlev et al. (2000o), 94. Masetti et al. (2000b), 95. Hurlev et al. (2000p), 96. Price et al. (2000b), 97. Hurlev et al. (2000a), 98. Price et al. (2002f), 99. Hurlev et al. (2000r), 100. Fvnbo et al. (2001b), 101. Hurlev et al. (2000s), 102. Castro Cerón et al. (2002a), 103. Hurley et al. (2000t), 104. Henden et al. (2000b), 105. Hurlev et al. (2000u), 106. Pedersen et al. (2006), 107. Vreeswiik et al. (2000), 
108. Hurley et al. (2000v), 109. Price et al. (2000c), 110. Hurley et al. (2000w), 111. Zhu (2000), 112. Barraud et al. (2003), 113. Masetti et al. (2001), 114. Sakamoto et al. (2005b), 115. Zhu (2001), 116. Rol et al. (2001), 117. Jha et al. (2001), 118. Galama et al. (2003), 119. Hurley et al. (2001b), 120. Oksanen et al. (2001), 121. Price et al. (2001c), 122. Hurley et al. (2001c), 123. Price et al. (2001d), 124. Andersen et al. (2001), 125. Price et al. (2002e), 126. Akerlof et al. (2001), 127. Heise et al. (2001), 128. Mohan et al. (2001), 129. Infante et al. (2001), 130. Greiner et al. (2003a), 131. Lee et al. (2001), 132. Vreeswijk et al. (2006), 133. Holland et al. (2002), 134. Saracco et al. (2001), 135. Hiorth et al. (2003), 136. Berger et al. (2002), 137. Berger et al. (2007a), 138. Castro Cerón et al. (2002b), 139. Lee et al. (2002), 140. Pavlenko \& Rumyantsev (2002), 141. Price et al. (2002a), 142. Monnelly et al. (2002), 143. Hurley et al. (2002b), 144. Masetti et al. (2003), 145. Levan et al. (2005), 146. Castro-Tirado et al. (2002a), 147. Hurley et al. (2002c), 148. Price et al. (2002b), 149. Amati et al. (2004), 150. Hurley et al. (2002d), 151. Henden \& Nelson (2002), 152. Hurlev et al. (2002e), 153. Gorosabel et al. (2002a), 154. Hurlev et al. (2002g), 155. Gorosabel et al. (2002b), 156. Hurlev et al. (2002f), 157. Gorosabel et al. (2002c), 158. Kilmartin \& Gilmore (2002), 159. Piccioni et al. (2002), 160. Price et al. (2002g), 161. Laursen \& Stanek (2003), 162. Jakobsson et al. (2005), 163. Levan et al. (2002), 164. Price et al. (2002d), 165. Soderberg et al. (2007), 166. Salamanca et al. (2002), 167. Holland et al. (2003), 168. Hurley et al. (2002h), 169. Castro-Tirado et al. (2002b) 170. Schaefer et al. (2002a), 171. Hurley et al. (2002i), 172. Bloom \& Falco (2002), 173. Fox \& Price (2002), 174. Schaefer et al. (2002b), 175. Yoshida et al. (2002), 176. Nvsewander et al. (2002b), 177. Vreeswijk et al. (2003), 178. Nvsewander et al. (2006), 179. Mereghetti et al. (2003a), 180. Castro-Tirado et al. (2002c), 181. Levan et al. (2006a), 182. Götz et al. (2003), 183. Fox et al. (2003a), 184. Hurlev et al. (2003a), 185. Nysewander et al. (2003), 186. Klose et al. (2004), 187. Mereghetti et al. (2003b), 188. Castro-Tirado et al. (2003), 189. Vreeswijk et al. (2004), 190. Gilmore et al. (2003), 191. Lamb et al. (2003), 192. Hurley et al. (2003b), 193. Buffington et al. (2006), 194. Martini et al. (2003), 195. Maiorano et al. (2006), 196. Tiengo et al. (2003), 197. Greiner et al. (2003b), 198. Resmi et al. (2005), 199. Hurlev et al. (2003c), 200. Schaefer et al. (2003), 201. Hurlev et al. (2003d), 202. Lipunov et al. (2003), 203. Henden et al. (2003), 204. Rykoff et al. (2004a), 205. Jakobsson et al. (2004), 206. Butler et al. (2004a), 207. Rau et al. (2005), 208. Rau et al. (2004), 209. Butler et al. (2005), 210. Bond (2003), 211. Pugliese et al. (2005), 212. Fox \& Hunt (2003), 213. Ibrahimov et al. (2003), 214. Guziv et al. (2003), 215. Butler et al. (2003), 216. Chen et al. (2003), 217. Hurlev et al. (2003e), 218. Silvev et al. (2003), 219. Ghisellini et al. (2006), 220. Prochaska et al. (2003), 221. Cobb et al. (2004), 222. Melandri et al. (2006a), 223. Fox et al. (2003b), 224. Moran et al. (2005), 225. Masetti et al. (2004), 226. Filliatre et al. (2006), 227. Mereghetti et al. (2005a), 228. de Ugarte et al. (2004a), 229. Filliatre et al. (2005), 230. Dullighan et al. (2004), 231. Gorosabel et al. (2004a), 232. Fugazza et al. (2004), 233. Barraud et al. (2004), 234. de Ugarte Postigo et al. (2004b), 235. http://space.mit.edu/HETE/Bursts/GRB040810/, 236. Price et al. (2004), 237. Urata et al. (2004), 238. Gorosabel et al. (2004b), 239. Butler et al. (2004b), 240. Stratta et al. (2007a), 241. Butler et al. (2004c), 242. Arimoto et al. (2007), 243. Kosugi et al. (2004), 244. Fenimore et al. (2004), 245. Wiersema et al. (2004), 246. Huang et al. (2005), 247. Galassi et al. (2004), 248. Butler et al. (2004d), 249. Stanek et al. (2005), 250. http://space.mit.edu/HETE/Bursts/GRB041016/, 251. Kuroda et al. (2004), 252. Dezalay et al. 
(2004), 253. Cenko et al. (2004), 254. Sakamoto et al. (2008), 255. Bavliss et al. (2004), 256. McBreen et al. (2006), 257. Hearty et al. (2004), 258. Nysewander et al. (2004), 259. Evans et al. (2007), 260. Berger et al. (2005c), 261. Rykoff et al. (2004b), 262. Rykoff (2004a), 263. Rykoff (2004b), 264. Hill et al. (2006), 265. de Ugarte Postigo et al. (2005), 266. Hurley et al. (2005a), 267. Li et al. (2005), 268. Gehrels et al. (2009), 269. Berger et al. (2005d), 270. Monfardini et al. (2005a), 271. Lamb et al. (2005), 272. Cenko \& Fox (2005a), 273. Smith et al. (2005), 274. Levan et al. (2006c), 275. Berger \& Gonzalez (2005), 276. D'Avanzo et al. (2005a), 277. Pellizza et al. (2006), 278. Nvsewander et al. (2005), 279. D'Avanzo et al. (2005b), 280. Kelson \& Berger (2005). 281. Berger \& Mulchaev (2005), 282. Still et al. (2005), 283. Jakobsson et al. (2006a), 284. Huang et al. (2007), 285. Tristram et al. (2005a), 286. de Pasquale et al. (2006b), 287. Berger et al. (2005e), 288. Sakamoto et al. (2005a), 289. Berger et al. (2005f), 290. de Ugarte Postigo et al. (2007a), 291. Cenko \& Fox (2005b), 292. Kosugi et al. (2005), 293. Cenko et al. (2005), 294. Holland et al. (2007b) 295. Berger et al. (2005b), 296. Monard \& Price (2005), 297. Cenko \& Fox (2005c), 298. McGowan et al. (2005), 299. Götz \& Mereghetti (2005), 300. Hurkett et al. (2005), 301. Prochaska et al. (2005a), 302. Yost et al. (2006), 303. Mereghetti et al. (2005b), 304. Cenko \& Fox (2005d), 305. Berger et al. (2006a), 306. Hurkett et al. (2006), 307. Fynbo et al. (2005a), 308. Prigozhin et al. (2005), 309. Campana et al. (2005), 310. Gorosabel et al. (2005a), 311. Götz et al. (2005a), 312. Guidorzi et al. (2005b), 313. Götz et al. (2005b), 314. Chiang et al. (2005), 315. Foley et al. (2005a), 316. Blustin et al. (2006a), 317. Monfardini et al. (2005b), 318. Berger \& Becker (2005), 319. Grupe et al. (2006), 320. Halpern et al. (2005b), 321. Götz et al. (2005c), 322. Mangano et al. (2005), 323. Gorosabel et al. (2005b), 324. de Pasquale et al. (2005), 325. de Pasquale et al. (2006c), 326. Monfardini et al. (2005c), 327. Bikmaev et al. (2005b), 328. Götz et al. (2005d), 329. Klose et al. (2005), 330. Covino et al. (2005), 331. Rol et al. (2007a), 332. MacLeod et al. (2005), 333. Antonelli et al. (2006), 334. Haislip \& Reichart (2005a), 335. Chen et al. (2005), 336. Pandey et al. (2006a), 337. de Pasquale et al. (2007a), 338. Rykoff et al. (2006), 339. Fynbo et al. (2005b), 340. Oates et al. (2007), 341. Haislip \& Reichart (2005b), 342. Maetou et al. (2005), 343. Rykoff et al. (2005), 344. Jakobsson et al. (2006b), 345. Jensen et al. (2005), 346. Bikmaev et al. (2005c), 347. Prochaska et al. (2005b), 348. Cenko et al. (2006g), 349. Page et al. (2005), 350. Fynbo et al. (2005c), 351. Sollerman et al. (2007), 352. Mirabal et al. (2007a), 353. Halpern (2005). 354. Kawai et al. (2006), 355. Haislip et al. (2006), 356. Foley et al. (2005b), 357. Sharapov et al. (2005a), 358. Bloom (2005), 359. Malesani et al. (2006), 360. Brown et al. (2005a), 361. Guziv et al. (2005), 362. Durig \& Price (2005), 363. Tristram et al. (2005b), 364. Rumvantsev et al. (2005a), 365. Rumvantsev et al. (2005b), 366. Beardmore et al. (2005), 367. Berger et al. (2005g), 368. Soderberg et al. (2005), 369. Sharapov et al. (2005b), 370. Olive et al. (2005), 371. Burenin et al. (2005), 372. Brown et al. (2005b), 373. Nakagawa et al. (2006), 374. Rol et al. (2007b), 375. Hurley et al. (2005b), 376. Castro-Tirado et al. (2006a), 377. Misra et al. (2005), 378. Quimby et al. (2005), 379. Perley et al. (2006), 380. Tagliaferri et al. (2005), 381. Penprase et al. (2006), 382. Guidorzi et al. (2007b), 383. Goad et al. (2007), 384. Thoene et al. (2005), 385. Mereghetti et al. (2005c), 386. Page et al. (2006a), 387. Halpern (2006), 388. Retter et al. (2006), 389. Armstrong et al. (2006), 390. Zimmerman \& Tyagi (2006), 391. Oates et al. (2006), 392. Blustin et al. (2006b), 393. Swan et al. (2006), 394. Khamitov et al. (2006a), 395. Yanagisawa et al. (2006a), 396. Mereghetti et al. (2006a), 397. Perri et al. (2006), 398. Guidorzi et al. 
(2006a), 399. Piranomonte et al. (2006b), 400. Yanagisawa et al. (2006b), 401. Kocevski et al. (2006), 402. Jelínek et al. (2006a), 403. Cummings et al. (2006), 404. Palmer et al. (2006), 405. Prochaska et al. (2006), 406. Misra et al. (2007), 407. Götz \& Mereghetti (2006), 408. Nielsen et al. (2006), 409. Butler (2007), 410. Cenko et al. (2006c), 411. Cenko et al. (2006b), 412. Mereghetti \& Götz (2006a), 413. Kong (2006), 414. Khamitov et al. (2006b), 415. Fynbo et al. (2006a), 416. Hao et al. (2007), 417. Stanek et al. (2007), 418. Curran et al. (2007), 419. Cucchiara et al. (2006b), 420. Sharapov et al. (2006a), 421. Sharapov et al. (2006b), 422. Golenetskii et al. (2006a), 423. Guziv et al. (2006), 424. Mirabal et al. (2006), 425. Rol et al. (2006a), 426. Berger et al. (2006b), 427. Blustin \& Cummings (2006), 428. Andreev \& Pozanenko (2006), 429. Lamb et al. (2006), 430. Hunsberger et al. (2006), 431. D'Avanzo et al. (2006a), 432. Jelínek et al. (2006b), 433. Covino et al. (2006b), 434. Sharapov et al. (2006c), 435. Blustin et al. (2006c), 436. Vreeswijk \& Jaunsen (2006), 437. Chen et al. (2006), 438. MacLeod et al. (2006), 439. Thoene et al. (2006a), 440. Lopez-Sanchez et al. (2006), 441. Cobb (2006a), 442. Halpern \& Mirabal (2006), 443. Fox \& Cenko (2006), 444. Cucchiara et al. (2006c), 445. Hicken \& Garnavich (2006), 446. Ofek et al. (2007), 447. Fynbo et al. (2006b), 448. Thoene et al. (2006b), 449. Holland (2006), 450. Price (2006), 451. Melandri et al. (2006b), 452. Bloom et al. (2006b), 453. Milne (2006), 454. Klotz et al. (2006a), 455. Cenko et al. (2006d), 456. D'Avanzo et al. (2006b), 457. Dai et al. (2007), 458. Jensen et al. (2006), 459. Castro-Tirado et al. (2006b), 460. Tanvir et al. (2006), 461. Ferrero et al. (2006), 462. Sharapov et al. (2006d), 463. Ledoux et al. (2006), 464. Nysewander et al. (2007), 465. French et al. (2006), 466. Valle et al. (2006), 467. Stefanescu et al. (2006a), 468. Schady \& Moretti (2006), 469. Cenko \& Ofek (2006), 470. Jakobsson et al. (2006c), 471. Jakobsson et al. (2006d), 472. Fugazza et al. (2006a), 473. Pagani et al. (2006), 474. Morgan et al. (2006), 475. Thoene et al. (2006c), 476. Pandey et al. (2006b), 477. Rol \& Page (2006), 478. Bellm et al. (2006), 479. Hurley et al. (2006), 480. Schady (2006), 481. Cucchiara et al. (2006d), 482. Thoene et al. (2007a), 483. Levan et al. (2006d), 484. D'Avanzo et al. (2006c), 485. Mereghetti \& Gotz (2006b), 486. Wiersema et al. (2007), 487. Cenko \& Rau (2006), 488. Fugazza et al. (2006b), 489. Sovano et al. (2006), 490. Cenko et al. (2006e), 491. Rol et al. (2006b), 492. Wiersema et al. (2006), 493. Levan et al. (2007b), 494. Hafizov et al. (2006), 495. Melandri et al. (2006c), 496. Fox et al. (2006), 497. Holland \& Cucchiara (2006), 4989. Covino et al. (2006c), 499. D'Elia et al. (2006), 500. Stefanescu et al. (2006b), 501. Fynbo et al. (2006c), 502. Antoniuk et al. (2006), 503. Golenetskii et al. (2006b), 504. Page et al. (2006b), 505. Terra et al. (2006), 506. Götz et al. (2006), 507. Guidorzi et al. (2006b), 508. Cannizzo et al. (2006), 509. Jakobsson et al. (2006e), 510. Osip et al. (2006), 511. Mundell et al. (2007), 512. Updike et al. (2006), 513. Holland \& Moretti (2006), 514. Mereghetti et al. (2006b), 515. Berger (2006b) 516. Stamatikos et al. (2006), 517. Parsons et al. (2006), 518. Klotz et al. (2006b), 519. Cenko et al. (2006f), 520. Holland et al. (2006), 521. Thoene et al. (2006d), 522. Fynbo (2006), 523. Fynbo et al. (2006d), 524. Thoene et al. (2006e), 525. Bloom et al. (2006c), 526. Halpern et al. (2006), 527. Mereghetti et al. (2006c), 528. Halpern \& Armstrong (2006), 529. Perley et al. (2008), 530. Pozanenko et al. (2006), 531. Sakamoto et al. (2006), 532. Klotz et al. (2006c), 533. Cenko \& Fox (2006), 534. Berger (2006c), 535. Cobb (2006b), 536. Chen et al. (2007a), 537. Bovd \& Marshall (2007), 538. Jaunsen et al. (2007a), 539. Malesani et al. (2007b), 540. Fox et al. (2007a), 541. Cenko \& Fox (2007), 542. Bellm et al. (2008), 543. Vetere et al. (2007a), 544. Malesani et al. (2007c), 545. Cucchiara et al. 
(2007b), 546. Halpern \& Mirabal (2007), 547. D'Avanzo et al. (2007), 548. Yoshida et al. (2007a), 549. Mirabal et al. (2007b), 550. Rol et al. (2007c), 551. Thoene et al. (2007b), 552. Klotz et al. (2007a), 553. Jaunsen et al. (2007b), 554. Rol et al. (2007d), 555. Vergani et al. (2007), 556. Paizis et al. (2007), 557. Mereghetti et al. (2007a), 558. Halpern \& Armstrong (2007), 559. Jaunsen et al. (2007c), 560. Cobb (2007a), 561. Markwardt et al. (2007e), 562. Grupe et al. (2007a), 563. Jakobsson et al. (2007a), 564. Berger et al. (2007d), 565. Prieto et al. (2007), 566. Cenko et al. (2007b), 567. Pozanenko et al. (2007), 568. de Ugarte Postigo et al. (2007b), 569. Guidorzi et al. (2007c), 570. Cannizzo et al. (2007), 571. Thoene et al. (2007c), 572. Pagani et al. (2007a), 573. Jakobsson et al. (2007b), 574. Vetere et al. (2007b), 575. Fox et al. (2007b) , 576. Terra et al. (2007), 577. Hattori et al. (2007), 578. Shakhovskoy et al. (2007), 579. Cenko et al. (2007c), 580. Swan et al. (2007), 581. de Ugarte Postigo et al. (2007c), 582. Thoene et al. (2007d), 583. Rykoff et al. (2007), 584. Updike et al. (2007), 585. Cenko et al. (2007d), 586. Grupe et al. (2007b), 587. Starling et al. (2007b), 588. Sbarufatti et al. (2007), 589. Malesani et al. (2007d), 590. Holland et al. (2007c), 591. Markwardt et al. (2007a), 592. Melandri et al. (2007), 593. Barthelmy et al. (2007), 594. Grupe et al. (2007c), 595. Ziaeepour et al. (2007b), 596. Palmer et al. (2007a), 597. Malesani et al. (2007e), 598. Golenetskii et al. (2007a), 599. Feroci et al. (2007), 600. Cenko \& Rau (2007), 601. Cummings et al. (2007a), 602. Prochaska et al. (2007a), 603. Berger \& Murphy (2007), 604. Stamatikos et al. (2007a), 605. Schady \& Parsons (2007), 606. Fenimore et al. (2007), 607. Stefanescu et al. (2007), 608. Thoene et al. (2007e), 609. Markwardt et al. (2007b), 610. Krimm \& Stratta (2007), 611. Cobb (2007b), 612. Markwardt et al. (2007c), 613. Klotz et al. (2007b), 614. Cummings et al. (2007b), 615. Fvnbo et al. (2007), 616. Grupe et al. (2007d), 617. Jaunsen et al. (2007d), 618. Racusin \& Barthelmy (2007), 619. Cobb (2007c), 620. Mereghetti et al. (2007b), 621. Bloom et al. (2007b), 622. de Pasquale et al. (2007b), 623. Schady et al. (2007), 624. Perley et al. (2007b), 625. Moretti et al. (2007), 626. Rumyantsev \& Pozanenko (2007), 627. Guidorzi et al. (2007d). 628. Roy et al. (2007e), 629. Prochaska et al. (2007b), 630. Markwardt et al. (2007d), 631. Stern et al. (2007), 632. Im et al. (2007), 633. Marshall et al. (2007c), 634. Iizuka \& Maeno (2007), 635. Palmer et al. (2007b), 636. Kann et al. (2007), 637. Krimm et al. (2007), 638. Xin et al. (2007b), 639. Holland et al. (2007d), 640. Jakobsson et al. (2007c), 641. Xin et al. (2007c), 642. Sakamoto et al. (2007), 643. Terada et al. (2007), 644. Pagani et al. (2007b), 645. Minezaki et al. (2007), 646. Racusin et al. (2007), 647. Xin et al. (2007d), 648. Grupe et al. (2007e), 649. Stroh et al. (2007), 650. Ledoux et al. (2007), 651. Lee et al. (2007), 652. McBreen et al. (2007b), 653. Perri et al. (2007b), 654. Milne (2007), 655. Stratta et al. (2007c), 656. Chen et al. (2007b), 657. Ukwatta et al. (2007), 658. Bloom (2007), 659. Cummings et al. (2007c), 660. Yoshida et al. (2007b), 661. Stamatikos et al. (2007b), 662. Cenko et al. (2007e) 


\section{REFERENCES}

Akerlof, C., McKay, T., Rykoff, E., \& Smith, D., 2001, GRB Coordinates Network, 1115, 1

Amati, L., et al. 2004, A\&A, 426, 415

Andersen, M. I., et al. 2000, A\&A, 364, L54

Andersen, M. I., Jensen, B. L., Hjorth, J., Pedersen, H., Kjeldsen, H., Gorosabel, J., \& Fynbo, J. U. 2001, GRB Coordinates Network, 1080, 1

Andreev, M., \& Pozanenko, A. 2006, GRB Coordinates Network, 4844, 1

Antonelli, L. A., et al. 1999, A\&AS, 138, 435

Antonelli, L. A., et al. 2006, A\&A, 456, 509

Antoniuk, K., Rumyantsev, V., \& Pozanenko, A. 2006, GRB Coordinates Network, 5647, 1

Arimoto, M., et al. 2007, PASJ, 59, 695

Armstrong, E., Halpern, J. P., Tyagi, S., \& Zimmerman, N. 2006, GRB Coordinates Network, 4427, 1

Barbier, L., et al. 2007, GRB Coordinates Network, 6623, 1

Barraud, C., et al. 2003, A\&A, 400, 1021

Barraud, C., et al. 2004, GRB Coordinates Network, 2620, 1

Barthelmy, S. D., et al. 2007, GRB Coordinates Network, 6622, 1

Bartolini, C., Gualandi, R., Guarnieri, A., Piccioni, A., Palazzi, E., Pian, E., \& Masetti, N. 2000, GRB Coordinates Network, 714, 1

Bayliss, M., Nysewander, M., Foster, A., Reichart, D., \& Moran, J. 2004, GRB Coordinates Network, 2864, 1

Beardmore, A. P., et al. 2005, GRB Coordinates Network, 4162, 1

Bellm, E., et al. 2006, GRB Coordinates Network, 5418, 1

Bellm, E. C., et al. 2008, ApJ, 688, 491

Berger, E., et al. 2002, ApJ, 581, 981 
Berger, E. 2005, GRB Coordinates Network, 3801, 1

Berger, E., \& Becker, G. 2005, GRB Coordinates Network, 3520, 1

Berger, E., \& Boss, A. 2005, GRB Coordinates Network, 4323, 1

Berger, E., \& Fox, D. 2005, GRB Coordinates Network, 4316, 1

Berger, E., \& Gonzalez, S. 2005, GRB Coordinates Network, 3048, 1

Berger, E., \& Mulchaey, J. 2005, GRB Coordinates Network, 3122, 1

Berger, E., et al. 2005a, Nature, 438, 988

Berger, E., Mulchaey, J., Morrell, N., \& Krzeminski, W. 2005b, GRB Coordinates Network, 3282,1

Berger, E., et al. 2005c, ApJ, 629, 328

Berger, E., Cenko, S. B., \& Kulkarni, S. R. 2005d, GRB Coordinates Network, 3088, 1

Berger, E., Oemler, G., \& Gladders, M. 2005e, GRB Coordinates Network, 3185, 1

Berger, E., Gladders, M., \& Oemler, G. 2005f, GRB Coordinates Network, 3201, 1

Berger, E., Roth, M., \& Gonzalez, S. 2005g, GRB Coordinates Network, 4098, 1

Berger, E. 2006a, GRB Coordinates Network, 5965, 1

Berger, E. 2006b, GRB Coordinates Network, 5754, 1

Berger, E. 2006c, GRB Coordinates Network, 5962, 1

Berger, E., Penprase, B. E., Cenko, S. B., Kulkarni, S. R., Fox, D. B., Steidel, C. C., \& Reddy, N. A. 2006a, ApJ, 642, 979

Berger, E., Kulkarni, S. R., Rau, A., \& Fox, D. B. 2006b, GRB Coordinates Network, 4815, 1

Berger, E. 2007, GRB Coordinates Network, 5995, 1

Berger, E., \& Murphy, D. 2007, GRB Coordinates Network, 6695, 1

Berger, E., \& Kaplan, D. L. 2007, GRB Coordinates Network, 6680, 1 
Berger, E., Fox, D. B., Kulkarni, S. R., Frail, D. A., \& Djorgovski, S. G. 2007a, ApJ, 660, 504

Berger, E., Morrell, N., \& Roth, M. 2007b, GRB Coordinates Network, 7154, 1

Berger, E., et al. 2007c, ApJ, 664, 1000

Berger, E., Modjaz, M., Garg, A., Kirshner, R., Challis, P., \& Rest, A. 2007d, GRB Coordinates Network, 6278, 1

Berger, E., Fox, D. B., \& Cucchiara, A. 2007e, GRB Coordinates Network, 6470, 1

Bikmaev, I., et al. 2005a, GRB Coordinates Network, 3797, 1

Bikmaev, I., et al. 2005b, GRB Coordinates Network, 3617, 1

Bikmaev, I., et al. 2005c, GRB Coordinates Network, 3831, 1

Bloom, J. S., Djorgovski, S. G., Kulkarni, S. R., \& Frail, D. A. 1998, ApJ, 507, L25

Bloom, J. S., \& Falco, E. 2002, GRB Coordinates Network, 1659, 1

Bloom, J. S., Berger, E., Kulkarni, S. R., Djorgovski, S. G., \& Frail, D. A. 2003, AJ, 125, 999

Bloom, J. S. 2005, GRB Coordinates Network, 3990, 1

Bloom, J. S., et al. 2006, ApJ, 638, 354

Bloom, J. S., Foley, R. J., Koceveki, D., \& Perley, D. 2006b, GRB Coordinates Network, 5217,1

Bloom, J. S., Perley, D. A., \& Chen, H. W. 2006c, GRB Coordinates Network, 5826, 1

Bloom, J. S. 2007, GRB Coordinates Network, 7105, 1

Bloom, J. S., et al. 2007a, ApJ, 654, 878

Bloom, J. S., Perley, D. A., Chen, H.-W., \& Prochaska, J. X. 2007b, GRB Coordinates Network, 6827, 1

Blustin, A. J., et al. 2006a, ApJ, 637, 901

Blustin, A. J., de Pasquale, M., Rosen, S. R., Cominsky, L., Norris, J., \& Gehrels, N. 2006b, GRB Coordinates Network, 4461, 1 
Blustin, A. J., Pagani, C., \& Kennea, J. 2006c, GRB Coordinates Network, 4964, 1

Blustin, A. J., \& Cummings, J. R. 2006, GRB Coordinates Network, 4824, 1

Bond, H. E. 2003, GRB Coordinates Network, 2329, 1

Boyd, P. T., \& Marshall, F. E. 2007, GRB Coordinates Network, 6006, 1

Brown, P. J., Morris, D., Huckle, H., Hancock, B., \& Gehrels, N. 2005a, GRB Coordinates Network, 4004, 1

Brown, P. J., Retter, A., Marshall, F., Smale, A., \& Gehrels, N. 2005b, GRB Coordinates Network, 4167, 1

Buffington, A., Band, D. L., Jackson, B. V., Hick, P. P., \& Smith, A. C. 2006, ApJ, 637, 880

Burenin, R., et al. 2005, GRB Coordinates Network, 4180, 1

Butler, N., et al. 2003, GRB Coordinates Network, 2429, 1

Butler, N., Dullighan, A., Ford, P., Ricker, G., Vanderspek, R., Hurley, K., Jernigan, J., \& Lamb, D. 2004a, Gamma-Ray Bursts: 30 Years of Discovery, 727, 435

Butler, N., et al. 2004b, GRB Coordinates Network, 2701, 1

Butler, N., et al. 2004c, GRB Coordinates Network, 2728, 1

Butler, N., Vanderspek, R., Marshall, H. L., Ford, P. G., Ricker, G. R., Lamb, D. Q., \& Garmire, G. P. 2004d, GRB Coordinates Network, 2808, 1

Butler, N. R., et al. 2005, ApJ, 621, 884

Butler, N. R. 2007, ApJ, 656, 1001

Campana, S., Pagani, C., Burrows, D. N., \& Gehrels, N. 2005, GRB Coordinates Network, 3441,1

Cannizzo, J. K., Barthelmy, S. D., Cummings, J., Page, K., Breeveld, A., Burrows, D., Roming, P., \& Gehrels, N. 2006, GCNR, 5, 1 (2006), 5, 1

Cannizzo, J. K., et al. 2007, GCNR, 52, 1 (2007), 52, 1

Castro, S. M., Diercks, A., Djorgovski, S. G., Kulkarni, S. R., Galama, T. J., Bloom, J. S., Harrison, F. A., \& Frail, D. A. 2000, GRB Coordinates Network, 605, 1 
Castro Cerón, J. M., et al. 2002a, A\&A, 393, 445

Castro Cerón, J. M., Gorosabel, J., Greiner, J., Klose, S., Snigula, J., \& Castro-Tirado, A. J. 2002b, GRB Coordinates Network, 1234, 1

Castro-Tirado, A. J., et al. 1997, IAU Circ., 6598, 2

Castro-Tirado, A. J., et al. 1998, GRB Coordinates Network, 173, 1

Castro-Tirado, A. J., Greiner, J., Phelps, S., Pian, E., \& Costa, E. 1999, GRB Coordinates Network, 336, 1

Castro-Tirado, A., Alises, M., \& Greiner, J. 2000, GRB Coordinates Network, 870, 1

Castro-Tirado, A. J., et al. 2001, A\&A, 370, 398

Castro-Tirado, A. J., Gorosabel, J., Castro Cerón, J. M., Nelson, B., \& Tristram, P. 2002a, GRB Coordinates Network, 1355, 1

Castro-Tirado, A. J., Klose, S., Wisotzki, L., Huferath, S., Greiner, J., Castro Cerón, J. M., \& Gorosabel, J. 2002b, GRB Coordinates Network, 1642, 1

Castro-Tirado, A. J., Gorosabel, J., Amado, P., \& Castro Cerón, J. M. 2002c, GRB Coordinates Network, 1775, 1

Castro-Tirado, A. J., et al. 2003, A\&A, 411, L315

Castro-Tirado, A. J., Bond, I., Kilmartin, P., de Ugarte Postigo, A., Gorosabel, J., Jelinek, M., \& Yock, P. 2005, GRB Coordinates Network, 3018, 1

Castro-Tirado, A. J., et al. 2006a, A\&A, 459, 763

Castro-Tirado, A. J., Amado, P., Negueruela, I., Gorosabel, J., Jelinek, M., \& de Ugarte Postigo, A. 2006b, GRB Coordinates Network, 5218, 1

Cenko, S. B., Fox, D. B., \& Gal-Yam, A. 2004, GRB Coordinates Network, 2848, 1

Cenko, S. B., \& Fox, D. B. 2005a, GRB Coordinates Network, 3014, 1

Cenko, S. B., \& Fox, D. B. 2005b, GRB Coordinates Network, 3231, 1

Cenko, S. B., \& Fox, D. B. 2005c, GRB Coordinates Network, 3299, 1

Cenko, S. B., \& Fox, D. B. 2005d, GRB Coordinates Network, 3349, 1 
Cenko, S. B., Kulkarni, S. R., Gal-Yam, A., \& Berger, E. 2005, GRB Coordinates Network, 3542,1

Cenko, S. B., \& Fox, D. B. 2006, GRB Coordinates Network, 5978, 1

Cenko, S. B., \& Ofek, E. O. 2006, GRB Coordinates Network, 5309, 1

Cenko, S. B., \& Rau, A. 2006, GRB Coordinates Network, 5512, 1

Cenko, S. B., Fox, D. B., \& Price, P. A. 2006a, GRB Coordinates Network, 5912, 1

Cenko, S. B., Ofek, E., Soderberg, A. M., Cucchiara, N., \& Fox, D. B. 2006b, GRB Coordinates Network, 4646, 1

Cenko, S. B., Moon, D.-S., \& Schmidt, B. P. 2006c, GRB Coordinates Network, 4636, 1

Cenko, S. B., Berger, E., Djorgovski, S. G., Mahabal, A. A., \& Fox, D. B. 2006d, GRB Coordinates Network, 5155, 1

Cenko, S. B., Ofek, E. O., \& Fox, D. B. 2006e, GRB Coordinates Network, 5529, 1

Cenko, S. B., Ofek, E. O., \& Kasliwal, M. 2006f, GRB Coordinates Network, 5770, 1

Cenko, S. B., et al. 2006, ApJ, 652, 490

Cenko, S. B., \& Fox, D. B. 2007, GRB Coordinates Network, 6028, 1

Cenko, S. B., \& Rau, A. 2007, GRB Coordinates Network, 6679, 1

Cenko, S. B., Rau, A., Berger, E., Price, P. A., \& Cucchiara, A. 2007a, GRB Coordinates Network, 6664, 1

Cenko, S. B., Gezari, S., Small, T., Fox, D. B., \& Chornock, R. 2007b, GRB Coordinates Network, 6322, 1

Cenko, S. B., Price, P. A., \& Berger, E. 2007c, GRB Coordinates Network, 6450, 1

Cenko, S. B., Fox, D. B., Cucchiara, A., Schmidt, B. P., Berger, E., Price, P. A., \& Roth, K. C. 2007d, GRB Coordinates Network, 6556, 1

Cenko, S. B., Fox, D. B., \& Ofek, E. 2007e, GRB Coordinates Network, 7123, 1

Chen, A. C., Ting, H. C., Lin, H. C., Huang, K. Y., Kinoshita, D., Ip, W. H., Urata, Y., \& Tamagawa, T. 2003, GRB Coordinates Network, 2436, 1 
Chen, H.-W., Prochaska, J. X., Bloom, J. S., \& Thompson, I. B. 2005, ApJ, 634, L25

Chen, B. A., Lin, C. S., Huang, K. Y., Ip, W. H., \& Urata, Y. 2006, GRB Coordinates Network, 4982, 1

Chen, H.-W., Dembicky, J., York, D., Lamb, D., McMillan, R., Ketzeback, B., \& Saurage, G. 2007a, GRB Coordinates Network, 5996, 1

Chen, I. C., Huang, K. Y., \& Urata, Y. 2007b, GRB Coordinates Network, 7083, 1

Chiang, P. S., Huang, K. Y., Ip, W. H., Urata, Y., Qiu, Y., \& Lou, Y. Q. 2005, GRB Coordinates Network, 3450, 1

Cobb, B. E., Bailyn, C. D., van Dokkum, P. G., Buxton, M. M., \& Bloom, J. S. 2004, ApJ, 608, L93

Cobb, B. E. 2006, GRB Coordinates Network, 5935, 1

Cobb, B. E. 2006a, GRB Coordinates Network, 5111, 1

Cobb, B. E. 2006b, GRB Coordinates Network, 5961, 1

Cobb, B. E. 2007a, GRB Coordinates Network, 6296, 1

Cobb, B. E. 2007b, GRB Coordinates Network, 6829, 1

Cobb, B. E. 2007c, GRB Coordinates Network, 6814, 1

Connaughton, V., et al. 1997, IAU Circ., 6683, 1

Covino, S., D’Avanzo, P., Malesani, D., Israel, G. L., Piranomonte, S., Tagliaferri, G., Chincarini, G., \& Stella, L. 2005, GRB Coordinates Network, 3616, 1

Covino, S., et al. 2006a, A\&A, 447, L5

Covino, S., Malesani, D., Antonelli, L. A., \& Fugazza, D. 2006b, GRB Coordinates Network, 4911,1

Covino, S., Malesani, D., \& Tagliaferri, G. 2006c, GRB Coordinates Network, 5604, 1

Cucchiara, A., Fox, D. B., Berger, E., \& Price, P. A. 2006a, GRB Coordinates Network, 5470,1

Cucchiara, A., Fox, D. B., \& Berger, E. 2006b, GRB Coordinates Network, 4729, 1 
Cucchiara, A., Price, P. A., Fox, D. B., Cenko, S. B., \& Schmidt, B. P. 2006c, GRB Coordinates Network, 5052, 1

Cucchiara, A., Brown, P., \& Moretti, A. 2006d, GRB Coordinates Network, 5445, 1

Cucchiara, A., Fox, D. B., Cenko, S. B., Berger, E., Price, P. A., \& Radomski, J. 2007a, GRB Coordinates Network, 6665, 1

Cucchiara, A., Fox, D. B., Cenko, S. B., \& Price, P. A. 2007b, GRB Coordinates Network, 6083,1

Cummings, J., et al. 2006, GRB Coordinates Network, 4608, 1

Cummings, J., et al. 2007a, GRB Coordinates Network, 6699, 1

Cummings, J. R., Barthelmy, S. D., Evans, P., \& Page, K. 2007b, GCNR, 85, 1 (2007b), 85, 1

Cummings, J. R., Moretti, A., \& de Pasquale, M. 2007c, GCNR, 109, 1 (2007c), 109, 1

Curran, P. A., et al. 2007, A\&A, 467, 1049

Cusumano, G., Burrows, D. N., Hunsberger, S., Pagani, C., La Parola, V., \& Mangano, V. 2005, GRB Coordinates Network, 4326, 1

Dai, X., Halpern, J. P., Morgan, N. D., Armstrong, E., Mirabal, N., Haislip, J. B., Reichart, D. E., \& Stanek, K. Z. 2007, ApJ, 658, 509

D'Avanzo, P., et al. 2005a, GRB Coordinates Network, 3064, 1

D'Avanzo, P., et al. 2005b, GRB Coordinates Network, 3089, 1

D’Avanzo, P., Israel, G. L., \& Cosentino, R. 2006a, GRB Coordinates Network, 4890, 1

D’Avanzo, P., Piranomonte, S., \& Magazzu, G. 2006b, GCN Coordinates Network, 5151, 1

D’Avanzo, P., Covino, S., \& Antonelli, L. A. 2006c, GRB Coordinates Network, 5475, 1

D’Avanzo, P., Magazzu, A., de Gurtubai, A. G., Antonelli, L. A., \& Sakamoto, T. 2007, GRB Coordinates Network, 6108, 1

D'Avanzo, P., et al. 2008, in preparation

D'Elia, V., et al. 2006, GRB Coordinates Network, 5637, 1 
de Pasquale, M., Boyd, P., Blustin, A., Nousek, J., \& Voges, W. 2005, GRB Coordinates Network, 3559, 1

de Pasquale, M., et al. 2006a, A\&A, 455, 813

de Pasquale, M., et al. 2006b, MNRAS, 365, 1031

de Pasquale, M., et al. 2006c, MNRAS, 370, 1859

de Pasquale, M., et al. 2007a, MNRAS, 377, 1638

de Pasquale, M., Stomatikos, M., \& Page, K. 2007b, GCNR, 86, 1 (2007b), 86, 1

de Ugarte, A., Sota, A., Gorosabel, J., Castro-Tirado, A. J., \& Castro Cerón, J. M. 2004a, GRB Coordinates Network, 2571, 1

de Ugarte Postigo, A., Tristram, P., Gorosabel, J., Yock, P., \& Castro-Tirado, A. J. 2004b, GRB Coordinates Network, 2621, 1

de Ugarte Postigo, A., Sota, A., Jelinek, M., Gorosabel, J., Castro-Tirado, A. J., \& Castro Cerón, J. M. 2005, GRB Coordinates Network, 2957, 1

de Ugarte Postigo, A., et al. 2007a, A\&A, 462, L57

de Ugarte Postigo, A., Jelinek, M., Tristram, P., Bond, I., Yock, P., Hearnshaw, J., \& Castro-Tirado, A. J. 2007b, GRB Coordinates Network, 6321, 1

de Ugarte Postigo, A., Castro-Tirado, A. J., Gonzalez, C., \& Cabrera, A. 2007c, GRB Coordinates Network, 6480, 1

Dezalay, J. P. et al. 2004, GRB Coordinates Network, 2839, 1

Diercks, A. H., et al. 1998, ApJ, 503, L105

Diercks, A., \& Cotoros, I. 2000, GRB Coordinates Network, 692, 1

Djorgovski, S. G., et al. 1997, Nature, 387, 876

Djorgovski, S. G., Kulkarni, S. R., Goodrich, R., Frail, D. A., \& Bloom, J. S. 1998, GRB Coordinates Network, 137, 1

Djorgovski, S. G., Kulkarni, S. R., Bloom, J. S., \& Frail, D. A. 1999, GRB Coordinates Network, 289, 1 
Djorgovski, S. G., Frail, D. A., Kulkarni, S. R., Bloom, J. S., Odewahn, S. C., \& Diercks, A. 2001, ApJ, 562, 654

Djorgovski, S. G., Bloom, J. S., \& Kulkarni, S. R. 2003, ApJ, 591, L13

Dodonov, S. N., Afanasiev, V. L., Sokolov, V. V., Moiseev, A. V., \& Castro-Tirado, A. J. 1999, GRB Coordinates Network, 475, 1

Donaghy, T. Q., et al. 2006, ArXiv Astrophysics e-prints, arXiv:astro-ph/0605570

Dullighan, A., et al. 2004, GRB Coordinates Network, 2588, 1

Durig, D. T., \& Price, A. 2005, GRB Coordinates Network, 4023, 1

Eldridge, J. J. 2007, MNRAS, 377, L29

Evans, P. A., et al. 2007, A\&A, 469, 379

Fenimore, E. E., et al. 2004, GRB Coordinates Network, 2735, 1

Fenimore, E., et al. 2007, GRB Coordinates Network, 6724, 1

Feroci, M., et al. 2007, GRB Coordinates Network, 6668, 1

Ferrero, P., et al. 2006, GRB Coordinates Network, 5489, 1

Filliatre, P., et al. 2005, A\&A, 438, 793

Filliatre, P., et al. 2006, A\&A, 448, 971

Foley, R. J., Chen, H.-W., Bloom, J., \& Prochaska, J. X. 2005a, GRB Coordinates Network, 3483,1

Foley, R. J., Chen, H.-W., Bloom, J. S., \& Prochaska, J. X. 2005b, GRB Coordinates Network, 3949, 1

Fox, D. W., \& Price, P. A. 2002, GRB Coordinates Network, 1671, 1

Fox, D. B., \& Hunt, M. P. 2003, GRB Coordinates Network, 2365, 1

Fox, D. W., Price, P. A., Herter, T., Appleton, P., \& Cotter, G. 2003a, GRB Coordinates Network, 1857, 1

Fox, D. B., Soderberg, A. M., \& Berger, E. 2003b, GRB Coordinates Network, 2499, 1

Fox, D. B., et al. 2005, Nature, 437, 845 
Fox, D. B., \& Cenko, S. B. 2006, GRB Coordinates Network, 5044, 1

Fox, D. B., Rau, A., \& Ofek, E. O. 2006, GRB Coordinates Network, 5597, 1

Fox, D. B., Berger, E., Price, P. A., \& Cenko, S. B. 2007a, GRB Coordinates Network, 6071, 1

Fox, D. B., Price, P. A., \& Berger, E. 2007b, GRB Coordinates Network, 6420, 1

French, J., Melady, G., Jelinek, M., de Ugarte Postigo, A., \& Kubanek, P. 2006, GRB Coordinates Network, 5247, 1

Frontera, F., Amati, L., Feroci, M., \& Costa, E. 1998, GRB Coordinates Network, 167, 1

Fugazza, D., et al. 2004, GRB Coordinates Network, 2617, 1

Fugazza, D., Malesani, D., \& Covino, S. 2006a, GRB Coordinates Network, 5347, 1

Fugazza, D., et al. 2006b, GRB Coordinates Network, 5513, 1

Fynbo, J. U., et al. 2001a, A\&A, 369, 373

Fynbo, J. U., et al. 2001b, A\&A, 373, 796

Fynbo, J. P. U., et al. 2005a, GRB Coordinates Network, 3460, 1

Fynbo, J. P. U., et al. 2005b, GRB Coordinates Network, 3749, 1

Fynbo, J. P. U., et al. 2005c, GRB Coordinates Network, 3874, 1

Fynbo, J. P. U. 2006, GRB Coordinates Network, 5818, 1

Fynbo, J. P. U., Gorosabel, J., Jensen, B. L., \& Naeraenen, J. 2006a, GRB Coordinates Network, 4677, 1

Fynbo, J. P. U., et al. 2006b, Nature, 444, 1047

Fynbo, J. P. U., et al. 2006c, GRB Coordinates Network, 5651, 1

Fynbo, J. P. U., Malesani, D., Thoene, C. C., Vreeswijk, P. M., Hjorth, J., \& Henriksen, C. 2006d, GRB Coordinates Network, 5809, 1

Fynbo, J. P. U., et al. 2007, GRB Coordinates Network, 6803, 1

Galama, T., et al. 1997, Nature, 387, 479 
Galama, T., et al., 1998, GRB Coordinates Network, 62, 1

Galama, T. J., et al. 1999, GRB Coordinates Network, 388, 1

Galama, T. J., et al. 2003, ApJ, 587, 135

Galassi, M., et al. 2004, GRB Coordinates Network, 2770, 1

Gehrels, N., et al. 2005, Nature, 437, 851

Gehrels, N., et al. 2009, in preparation

Gendre, B., Corsi, A., \& Piro, L. 2006, A\&A, 455, 803

Ghisellini, G., Ghirlanda, G., Mereghetti, S., Bosnjak, Z., Tavecchio, F., \& Firmani, C. 2006, MNRAS, 372, 1699

Gilmore, A., Kilmartin, P., \& Henden, A. 2003, GRB Coordinates Network, 1949, 1

Goad, M. R., et al. 2007, A\&A, 468, 103

Golenetskii, S., Aptekar, R., Mazets, E., Pal'Shin, V., Frederiks, D., \& Cline, T. 2006a, GRB Coordinates Network, 4763, 1

Golenetskii, S., Aptekar, R., Mazets, E., Pal'Shin, V., Frederiks, D., \& Cline, T. 2006b, GRB Coordinates Network, 5689, 1

Golenetskii, S., Aptekar, R., Mazets, E., Pal'Shin, V., Frederiks, D., \& Cline, T. 2007a, GRB Coordinates Network, 6615, 1

Golenetskii, S., Aptekar, R., Mazets, E., Pal'Shin, V., Frederiks, D., \& Cline, T. 2007b, GRB Coordinates Network, 6671, 1

Gorosabel, J., et al. 1999, A\&AS, 138, 455

Gorosabel, J., et al. 2000a, GRB Coordinates Network, 563, 1

Gorosabel, J., Pascual, S., Gallego, J., Zamorano, J., Castro-Tirado, A. J., Castro Cerón, J. M., Klose, S., \& Greiner, J. 2000b, GRB Coordinates Network, 735, 1

Gorosabel, J., Hjorth, J., Jensen, B. L., Pedersen, H., Jakobsson, P., Fynbo, J., \& Andersen, M. I. 2002a, GRB Coordinates Network, 1429, 1

Gorosabel, J., Castro Cerón, J. M., Castro-Tirado, A. J., Guijarro, A., Hoyo, F., Pedraz, S., \& Wolf, C. 2002b, GRB Coordinates Network, 1466, 1 
Gorosabel, J., Castro Cerón, J. M., Castro Tirado, A. J., Guijarro, A., Hoyo, F., Pedraz, S., \& Wolf, C. 2002c, GRB Coordinates Network, 1467, 1

Gorosabel, J., Jelinek, M., Mendez, J., Ruiz-Lapuente, P., Castro Cerón, J. M., Ugarte, P., \& Castro-Tirado, A. J. 2004a, GRB Coordinates Network, 2592, 1

Gorosabel, J., de Ugarte Postigo, A., Miranda, L. F., Castro-Tirado, A. J., Jelinek, M., Pereira, C. B., Castro Cerón, J. M., \& Aceituno, J. 2004b, GRB Coordinates Network, 2675,1

Gorosabel, J., et al. 2005a, GRB Coordinates Network, 3425, 1

Gorosabel, J., et al. 2005b, GRB Coordinates Network, 3565, 1

Götz, D., et al. 2003, A\&A, 409, 831

Götz, D., \& Mereghetti, S. 2005, GRB Coordinates Network, 3329, 1

Götz, D., Mereghetti, S., Mowlavi, N., Shaw, S., Beck, M., \& Borkowski, J. 2005a, GRB Coordinates Network, 3430, 1

Götz, D., Mereghetti, S., Milano, I., Mowlavi, N., Shaw, S., Beck, M., \& Borkowski, J. 2005b, GRB Coordinates Network, 3446, 1

Götz, D., Mereghetti, S., Mowlavi, N., Shaw, S., Beck, M., Neronov, A., \& Borkowski, J. 2005c, GRB Coordinates Network, 3552, 1

Götz, D., Mereghetti, S., Shaw, S., Mowlavi, N., Beck, M., Soldi, S., \& Borkowski, J. 2005d, GRB Coordinates Network, 3607, 1

Götz, D., \& Mereghetti, S. 2006, GRB Coordinates Network, 4616, 1

Götz, D., Mereghetti, S., Paizis, A., Lazos, M., Mowlavi, N., Beck, M., \& Borkowski, J. 2006, GRB Coordinates Network, 5665, 1

Graham, J. F., Fruchter, A. S., Levan, A. J., Nysewander, M., Tanvir, N. R., Dahlen, T., Bersier, D., \& Pe'Er, A. 2007, GRB Coordinates Network, 6836, 1

Greiner, J., Pompei, E., Els, S., Pinfield, D., Brillant, S., Sterzik, M., Wolf, C., \& Antonelli, A. 1999, GRB Coordinates Network, 396, 1

Greiner, J., et al. 2003a, ApJ, 599, 1223

Greiner, J., Peimbert, M., Estaban, C., Kaufer, A., Jaunsen, A., Smoke, J., Klose, S., \& Reimer, O. 2003b, GRB Coordinates Network, 2020, 1 
Groot, P. J., et al. 1997a, IAU Circ., 6616, 2

Groot, P. J. et al. 1997b, GRB Coordinates Network, 17, 1

Groot, P. J., et al. 1998a, ApJ, 493, L27

Groot, P. J., et al. 1998b, ApJ, 502, L123

Grupe, D., et al. 2006, ApJ, 645, 464

Grupe, D., et al. 2007a, GCNR, 43, 2 (2007), 43, 2

Grupe, D., et al. 2007b, GCNR, 65, 1 (2007), 65, 1

Grupe, D., et al. 2007c, GCNR, 71, 1 (2007), 71, 1

Grupe, D., Barthelmy, S. D., Palmer, D. M., Burrows, D. N., \& Gehrels, N. 2007d, GCNR, 83,1 (2007), 83, 1

Grupe, D., Cummings, J. R., Schady, P., Barthelmy, S. D., Burrows, D. N., Roming, P., \& Gehrels, N. 2007e, GCNR, 107, 1 (2007), 107, 1

Guidorzi, C., et al. 2005a, GRB Coordinates Network, 4356, 1

Guidorzi, C., et al. 2005b, GRB Coordinates Network, 3437, 1

Guidorzi, C., et al. 2006a, GRB Coordinates Network, 4504, 1

Guidorzi, C., et al. 2006b, GRB Coordinates Network, 5670, 1

Guidorzi, C., et al. 2007a, GCNR, 77, 1 (2007), 77, 1

Guidorzi, C., et al. 2007b, A\&A, 463, 539

Guidorzi, C., et al. 2007c, GRB Coordinates Network, 6334, 1

Guidorzi, C., Romano, P., Moretti, A., Krimm, H., Barthelmy, S. D., Burrows, D. N., Roming, P., \& Gehrels, N. 2007d, GCNR, 89, 2 (2007), 89, 2

Guziy, S., et al. 2003, GRB Coordinates Network, 2391, 1

Guziy, S., Jelinek, M., Gorosabel, J., Castro-Tirado, A. J., de Ugarte Postigo, A., Flores, E. R., \& Vijanen, K. 2005, GRB Coordinates Network, 4025, 1

Guziy, S., et al. 2006, GRB Coordinates Network, 4771, 1 
Hafizov, B., Ibrahimov, M., \& Pozanenko, A. 2006, GRB Coordinates Network, 5567, 1

Haislip, J., \& Reichart, D. 2005a, GRB Coordinates Network, 3719, 1

Haislip, J., \& Reichart, D. 2005b, GRB Coordinates Network, 3780, 1

Haislip, J. B., et al. 2006, Nature, 440, 181

Halpern, J. P., et al. 2000, ApJ, 543, 697

Halpern, J. P. 2005, GRB Coordinates Network, 3900, 1

Halpern, J. P., Tonnesen, S., Tuttle, S., \& Mirabal, N. 2005a, GRB Coordinates Network, 4202,1

Halpern, J. P., Kemp, J., \& Mirabal, N. 2005b, GRB Coordinates Network, 3530, 1

Halpern, J. 2006, GRB Coordinates Network, 4614, 1

Halpern, J. P., \& Armstrong, E. 2006, GRB Coordinates Network, 5849, 1

Halpern, J. P., \& Mirabal, N. 2006, GRB Coordinates Network, 5034, 1

Halpern, J. P., Mirabal, N., \& Armstrong, E. 2006, GRB Coordinates Network, 5840, 1

Halpern, J. P., \& Mirabal, N. 2007, GRB Coordinates Network, 6082, 1

Halpern, J. P., \& Armstrong, E. 2007, GRB Coordinates Network, 6195, 1

Hammer, F., Flores, H., Schaerer, D., Dessauges-Zavadsky, M., Le Floc'h, E., \& Puech, M. 2006, A\&A, 454, 103

Hao, H., et al. 2007, ApJ, 659, L99

Harrison, F. A., et al. 1999, ApJ, 523, L121

Hattori, T., Aoki, K., \& Kawai, N. 2007, GRB Coordinates Network, 6441, 1

Hearty, F., et al. 2004, GRB Coordinates Network, 2916, 1

Heise, J., in't Zand, J. J. M., Kulkarni, S. R., \& Costa, E. 2001, GRB Coordinates Network, 1138, 1

Henden, A. 2000, GRB Coordinates Network, 658, 1 
Henden, A., Castro-Tirado, A. J., \& Castro Cerón, J. M. 2000a, GRB Coordinates Network, 621,1

Henden, A., Kaiser, D., Hohman, D., Pason, R., \& Aquino, B. 2000b, GRB Coordinates Network, 858, 1

Henden, A., \& Nelson, P. 2002, GRB Coordinates Network, 1397, 1

Henden, A., Silvay, J., Moran, J., Soule, R., Reichart, D., Schaefer, J., Canterna, R., \& Scoggins, B. 2003, GRB Coordinates Network, 2250, 1

Hicken, M., \& Garnavich, P. 2006, GRB Coordinates Network, 5070, 1

Hill, J. E., et al. 2006, ApJ, 639, 303

Hjorth, J., et al. 2002, ApJ, 576, 113

Hjorth, J., et al. 2003, ApJ, 597, 699

Holland, S. T., et al. 2002, AJ, 124, 639

Holland, S. T., et al. 2003, AJ, 125, 2291

Holland, S. T. 2006, GRB Coordinates Network, 5116, 1

Holland, S., \& Cucchiara, A. 2006, GRB Coordinates Network, 5603, 1

Holland, S. T., \& Moretti, A. 2006, GRB Coordinates Network, 5745, 1

Holland, S. T., Tueller, J., Starling, R. L. C., Page, K., Burrows, D. N., Nousek, J., Barthelmy, S., \& Gehrels, N. 2006, GCNR, 12, 2 (2006), 12, 2

Holland, S. T., de Pasquale, M., \& Markwardt, C. B. 2007a, GRB Coordinates Network, 7145,1

Holland, S. T., et al. 2007b, AJ, 133, 122

Holland, S. T., et al. 2007c, GCNR, 68, 1 (2007), 68, 1

Holland, S. T., et al. 2007d, GCNR, 94, 2 (2007), 94, 2

Huang, K. Y., et al. 2005, ApJ, 628, L93

Huang, K. Y., et al. 2007, ApJ, 654, L25

Hunsberger, S. D., Grupe, D., \& Marshall, F. 2006, GRB Coordinates Network, 4865, 1 
Hurkett, C., Page, K., Osborne, J. P., Zhang, B., Kennea, J., Burrows, D. N., \& Gehrels, N. 2005, GRB Coordinates Network, 3374, 1

Hurkett, C. P., et al. 2006, MNRAS, 368, 1101

Hurley, K., \& Cline, T. 1999, GRB Coordinates Network, 347, 1

Hurley, K., Cline, T., \& Mazets, E. 2000a, GRB Coordinates Network, 525, 1

Hurley, K., Cline, T., Frontera, F., Guidorzi, C., Montanari, E., Mazets, E., \& Golenetskii, S. 2000b, GRB Coordinates Network, 895, 1

Hurley, K., Cline, T., Mazets, E., \& Golenetskii, S. 2000c, GRB Coordinates Network, 865, 1

Hurley, K., Cline, T., \& Mazets, E. 2000d, GRB Coordinates Network, 529, 1

Hurley, K., et al. 2000e, ApJ, 534, L23

Hurley, K., Cline, T., Kouveliotou, C., Kippen, R. M., Mazets, E., \& Golenetskii, S. 2000f, GRB Coordinates Network, 601, 1

Hurley, K., Kippen, R. M., Barthelmy, S., \& Cline, T. 2000g, GRB Coordinates Network, 616,1

Hurley, K., Barthelmy, S., Kippen, R. M., \& Cline, T. 2000h, GRB Coordinates Network, 626,1

Hurley, K., Cline, T., \& Mazets, E. 2000i, GRB Coordinates Network, 642, 1

Hurley, K., Cline, T., Mazets, E., \& Golenetskii, S. 2000j, GRB Coordinates Network, 687, 1

Hurley, K., Cline, T., Mazets, E., \& Golenetskii, S. 2000k, GRB Coordinates Network, 711, 1

Hurley, K., Cline, T., Mazets, E., \& Golenetskii, S. 2000l, GRB Coordinates Network, 730, 1

Hurley, K., Golenetskii, S., Mazets, E., \& Cline, T. 2000m, GRB Coordinates Network, 761, 1

Hurley, K., Cline, T., Mazets, E., Golenetskii, S., Frontera, F., Montanari, E., \& Guidorzi, C. 2000n, GRB Coordinates Network, 736, 1 
Hurley, K., Cline, T., Mazets, E., \& Golenetskii, S. 2000o, GRB Coordinates Network, 770, 1

Hurley, K., Cline, T., Mazets, E., \& Golenetskii, S. 2000p, GRB Coordinates Network, 779, 1

Hurley, K., Cline, T., Mazets, E., \& Golenetskii, S. 2000q, GRB Coordinates Network, 791, 1

Hurley, K., Mazets, E., Golenetskii, S., \& Cline, T. 2000r, GRB Coordinates Network, 801, 1

Hurley, K., Cline, T., Mazets, E., \& Golenetskii, S. 2000s, GRB Coordinates Network, 841, 1

Hurley, K., Cline, T., Mazets, E., \& Golenetskii, S. 2000t, GRB Coordinates Network, 855, 1

Hurley, K., Cline, T., \& Smith, D. 2000u, GRB Coordinates Network, 863, 1

Hurley, K., Cline, T., Mazets, E., \& Golenetskii, S. 2000v, GRB Coordinates Network, 888, 1

Hurley, K., Cline, T., Montanari, E., Guidorzi, C., Frontera, F., Mazets, E., \& Golenetskii, S. 2000w, GRB Coordinates Network, 899, 1

Hurley, K., Cline, T., Mazets, E., \& Golenetskii, S. 2001a, GRB Coordinates Network, 916, 1

Hurley, K., Guidorzi, C., Montanari, E., \& Frontera, F. 2001b, GRB Coordinates Network, 1013, 1

Hurley, K., Guidorzi, C., Montanari, E., \& Frontera, F. 2001c, GRB Coordinates Network, 1036, 1

Hurley, K., et al. 2002a, GRB Coordinates Network, 1409, 1

Hurley, K., et al. 2002b, GRB Coordinates Network, 1325, 1

Hurley, K., et al. 2002c, GRB Coordinates Network, 1376, 1

Hurley, K., et al. 2002d, GRB Coordinates Network, 1395, 1

Hurley, K., et al. 2002e, GRB Coordinates Network, 1417, 1 
Hurley, K., et al. 2002f, GRB Coordinates Network, 1454, 1

Hurley, K., et al. 2002g, GRB Coordinates Network, 1455, 1

Hurley, K., Mazets, E., Golenetskii, S., \& Cline, T. 2002h, GRB Coordinates Network, 1617, 1

Hurley, K., et al. 2002i, GRB Coordinates Network, 1653, 1

Hurley, K., et al. 2002j, GRB Coordinates Network, 1719, 1

Hurley, K., et al. 2003a, GRB Coordinates Network, 1854, 1

Hurley, K., et al. 2003b, GRB Coordinates Network, 1962, 1

Hurley, K., et al. 2003c, GRB Coordinates Network, 2135, 1

Hurley, K., et al. 2003d, GRB Coordinates Network, 2138, 1

Hurley, K., et al. 2003e, GRB Coordinates Network, 2443, 1

Hurley, K., et al. 2005a, GRB Coordinates Network, 2966, 1

Hurley, K., et al. 2005b, GRB Coordinates Network, 4172, 1

Hurley, K., et al. 2006, GRB Coordinates Network, 5407, 1

Ibrahimov, M. A., Asfandiyarov, I. M., Kahharov, B. B., Pozanenko, A., Rumyantsev, V., \& Beskin, G. 2003, GRB Coordinates Network, 2368, 1

Iizuka, R., \& Maeno, S. 2007, GRB Coordinates Network, 6900, 1

Im, M., Lee, I., \& Urata, Y. 2007, GRB Coordinates Network, 6920, 1

Infante, L., Garnavich, P. M., Stanek, K. Z., \& Wyrzykowski, L. 2001, GRB Coordinates Network, 1152, 1

Jakobsson, P., et al. 2004, A\&A, 427, 785

Jakobsson, P., et al. 2005, ApJ, 629, 45

Jakobsson, P., et al. 2006a, A\&A, 460, L13

Jakobsson, P., et al. 2006b, Gamma-Ray Bursts in the Swift Era, 836, 552 
Jakobsson, P., Vreeswijk, P., Fynbo, J. P. U., Hjorth, J., Starling, R., Kann, D. A., \& Hartmann, D. 2006c, GRB Coordinates Network, 5320, 1

Jakobsson, P., Fynbo, J. P. U., Hjorth, J., Nielsen, T. B., \& Holhjem, K. 2006d, GRB Coordinates Network, 5335, 1

Jakobsson, P., et al. 2006e, GRB Coordinates Network, 5782, 1

Jakobsson, P., Malesani, D., Thoene, C. C., Fynbo, J. P. U., Hjorth, J., Jaunsen, A. O., Andersen, M. I., \& Vreeswijk, P. M. 2007a, GRB Coordinates Network, 6283, 1

Jakobsson, P., et al. 2007b, GRB Coordinates Network, 6398, 1

Jakobsson, P., Vreeswijk, P. M., Hjorth, J., Malesani, D., Fynbo, J. P. U., \& Thoene, C. C. 2007c, GRB Coordinates Network, 6952, 1

Jaunsen, A. O., Malesani, D., Fynbo, J. P. U., Sollerman, J., \& Vreeswijk, P. M. 2007a, GRB Coordinates Network, 6010, 1

Jaunsen, A. O., Thoene, C. C., Fynbo, J. P. U., Hjorth, J., \& Vreeswijk, P. 2007b, GRB Coordinates Network, 6202, 1

Jaunsen, A. O., Fynbo, J. P. U., Andersen, M. I., \& Vreeswijk, P. 2007c, GRB Coordinates Network, 6216, 1

Jaunsen, A. O., et al. 2007d, GRB Coordinates Network, 6921, 1

Jelínek, M., et al. 2006a, A\&A, 454, L119

Jelinek, M., Guziy, S., Gonzalez-Perez, J. M., \& Castro-Tirado, A. J. 2006b, GRB Coordinates Network, 4920, 1

Jensen, B. L., et al. 1999, GRB Coordinates Network, 371, 1

Jensen, B. L., et al. 2000a, GRB Coordinates Network, 670, 1

Jensen, B. L., Pedersen, H., Hjorth, J., Gorosabel, J., dall, T. H., Fynbo, J. P. U., \& O’Toole, S. 2000b, GRB Coordinates Network, 679, 1

Jensen, B. L., et al. 2001, A\&A, 370, 909

Jensen, B. L., et al. 2005, GRB Coordinates Network, 3809, 1

Jensen, B. L., Hjorth, J., Fynbo, J., \& Naranen, J. 2006, GRB Coordinates Network, 5203, 1 
Jha, S., et al. 2001, ApJ, 554, L155

Johnson, S., et al. 2007, GRB Coordinates Network, 6218, 1

Kann, D. A., Hoegner, C., \& Filgas, R. 2007, GRB Coordinates Network, 6917, 1

Kawai, N., et al. 2006, Nature, 440, 184

Kelson, D., \& Berger, E. 2005, GRB Coordinates Network, 3101, 1

Khamitov, I., et al. 2006a, GRB Coordinates Network, 4494, 1

Khamitov, I., et al. 2006b, GRB Coordinates Network, 4720, 1

Kilmartin, P., \& Gilmore, A. 2002, GRB Coordinates Network, 1462, 1

Kippen, R. M., Preece, R. D., Giblin, T., Kouveliotou, C. 2007, GRB Coordinates Network, 344,1

Kippen, R. M. \& Woods, P. M. 2000, GRB Coordinates Network, 639, 1

Kjernsmo, K., Jaunsen, A., Saanum, O., Jensen, B. L., Hjorth, J., Pedersen, H., \& Gorosabel, J. 2000, GRB Coordinates Network, 533, 1

Klose, S., et al. 2000, ApJ, 545, 271

Klose, S., et al. 2004, AJ, 128, 1942

Klose, S., Stecklum, B., Fuhrmann, B., Ludwig, F., \& Greiner, J. 2005, GRB Coordinates Network, 3609, 1

Klotz, A., Boer, M., \& Atteia, J. L. 2006a, GRB Coordinates Network, 5134, 1

Klotz, A., Boer, M., \& Atteia, J. L. 2006b, GRB Coordinates Network, 5788, 1

Klotz, A., Boer, M., \& Atteia, J. L. 2006c, GRB Coordinates Network, 5980, 1

Klotz, A., Boer, M., \& Atteia, J. L. 2007a, GRB Coordinates Network, 6159, 1

Klotz, A., Boer, M., \& Atteia, J. L. 2007b, GRB Coordinates Network, 6787, 1

Kocevski, D., Bloom, J. S., \& McGrath, E. J. 2006, GRB Coordinates Network, 4528, 1

Kocevski, D., \& Bloom, J. S. 2007, GRB Coordinates Network, 7107, 1

Kong, A. 2006, GRB Coordinates Network, 4676, 1 
Kosugi, G., Kawai, N., Tajitsu, A., \& Furusawa, H. 2004, GRB Coordinates Network, 2726, 1

Kosugi, G., Kawai, N., Aoki, K., Hattori, T., Ohta, K., \& Yamada, T. 2005, GRB Coordinates Network, 3263, 1

Krimm, H. A., \& Stratta, G. 2007, GCNR, 82, 1 (2007), 82, 1

Krimm, H. A., Beardmore, A., Cummings, J. R., Barthelmy, S. D., Burrows, D. N., Gehrels, N., \& Roming, P. W. A. 2007, GCNR, 96, 2 (2007), 96, 2

Kulkarni, S. R., et al. 1999, Nature, 398, 389

Kuroda, D., Yanagisawa, K., \& Kawai, N. 2004, GRB Coordinates Network, 2818, 1

La Parola, V., et al. 2006, A\&A, 454, 753

Lamb, D. Q., et al. 2002, GRB Coordinates Network, 1403, 1

Lamb, D. Q., Nysewander, M., Reichart, D., York, D. G., Barentine, J., McMillan, R., Dembicky, J., \& Ketzeback, B. 2003, GRB Coordinates Network, 2139, 1

Lamb, D., et al. 2005, GRB Coordinates Network, 3019, 1

Lamb, D. Q., Nysewander, M., Hearty, F., Chen, H.-W., \& Reichart, D. E. 2006, GRB Coordinates Network, 5079, 1

Laursen, L. T., \& Stanek, K. Z. 2003, ApJ, 597, L107

Ledoux, C., Vreeswijk, P., Smette, A., Jaunsen, A., \& Kaufer, A. 2006, GRB Coordinates Network, 5237, 1

Ledoux, C., Jakobsson, P., Jaunsen, A. O., Thoene, C. C., Vreeswijk, P. M., Malesani, D., Fynbo, J. P. U., \& Hjorth, J. 2007, GRB Coordinates Network, 7023, 1

Lee, I., Im, M., \& Urata, Y. 2007, GRB Coordinates Network, 7033, 1

Le Floc'h, E., et al. 2002, ApJ, 581, L81

Lee, B. C., Tucker, D. L., Lamb, D. Q., vanden Berk, D. E., \& Neilsen, E. 2001, GRB Coordinates Network, 1175, 1

Lee, B. C., et al. 2002, GRB Coordinates Network, 1275, 1 
Levan, A., Burud, I., Fruchter, A., Rhoads, J., van den Berg, M., Homan, J., Rol, E., \& Tanvir, N. 2002, GRB Coordinates Network, 1517, 1

Levan, A., et al. 2005, ApJ, 624, 880

Levan, A., et al. 2006a, ApJ, 647, 471

Levan, A. J., et al. 2006b, ApJ, 648, L9

Levan, A. J., et al. 2006c, ApJ, 648, 1132

Levan, A. J., Tanvir, N. R., Rol, E., Fruchter, A., \& Adamson, A. 2006d, GRB Coordinates Network, 5455, 1

Levan, A. J., et al. 2007a, GRB Coordinates Network, 6603, 1

Levan, A. J., et al. 2007b, MNRAS, 378, 1439

Levan, A. J., et al. 2008, MNRAS, 384, 541

Li, W., Filippenko, A. V., Chornock, R., \& Jha, S. 2005, GRB Coordinates Network, 2968, 1

Lipunov, V., et al. 2003, GRB Coordinates Network, 2157, 1

Lopez-Sanchez, A. R., Garcia-Rojas, J., Jelinek, M., \& Castro-Tirado, A. J. 2006, GRB Coordinates Network, 5013, 1

MacLeod, C., Kirschbrown, J., Haislip, J., Nysewander, M., Crain, J. A., \& Reichart, D. 2005, GRB Coordinates Network, 3652, 1

MacLeod, C., et al. 2006, GRB Coordinates Network, 4999, 1

Maetou, M., et al. 2005, GRB Coordinates Network, 3785, 1

Maiorano, E., et al. 2006, A\&A, 455, 423

Malesani, D., Covino, S., Piranomonte, S., Antonelli, L. A., Chincarini, G., D'Avanzo, P., Stella, L., \& Tagliaferri, G. 2006, GRB Coordinates Network, 5821, 1

Malesani, D., et al. 2007a, A\&A, 473, 77

Malesani, D., Jaunsen, A. O., \& Vreeswijk, P. M. 2007b, GRB Coordinates Network, 6015, 1 
Malesani, D., Jakobsson, P., Vreeswijk, P. M., Jaunsen, A. O., \& Kann, D. A. 2007c, GRB Coordinates Network, 6055, 1

Malesani, D., Thoene, C. C., Fynbo, J. P. U., Tanvir, N. R., \& Tziamtzis, A. 2007d, GRB Coordinates Network, 6565, 1

Malesani, D., Jakobsson, P., Fynbo, J. P. U., Hjorth, J., \& Vreeswijk, P. M. 2007e, GRB Coordinates Network, 6651, 1

Mangano, V., et al. 2005, GRB Coordinates Network, 3564, 1

Markwardt, C., et al. 2007a, GRB Coordinates Network, 6596, 1

Markwardt, C., et al. 2007b, GRB Coordinates Network, 6748, 1

Markwart, C. B., Beardmore, A., Barthelmy, S. D., Burrows, D. N., Roming, P., \& Gehrels, N. 2007c, GCNR, 88, 1 (2007), 88, 1

Markwardt, C., Kennea, J., Mangano, V., Barthelmy, S. D., Burrows, D. N., Roming, P., \& Gehrels, N. 2007d, GCNR, 92, 1 (2007), 92, 1

Markwardt, C. B., Evans, P. A., Goad, M., \& Marshall, F. E. 2007, GCNR, 42, 3 (2007), 42,3

Marshall, F. E., Takeshima, T. 2000, GRB Coordinates Network, 58, 1

Marshall, F. E., Barthelmy, S. D., Burrows, D. N., Chester, M. M., Cummings, J., Evans, P. A., Roming, P., \& Gehrels, N. 2007a, GCNR, 80, 1 (2007), 80, 1

Marshall, F. E., Barthelmy, S. D., Brown, P. J., Burrows, D. N., Cummings, J., Roming, P., Starling, R., \& Gehrels, N. 2007b, GCNR, 81, 1 (2007), 81, 1

Marshall, F. E., Barthelmy, S. D., Burrows, D. N., Roming, P., Sbarufatti, B., \& Gejrels, N. 2007c, GCNR, 93, 1 (2007), 93, 1

Martini, P., Garnavich, P., \& Stanek, K. Z. 2003, GRB Coordinates Network, 1980, 1

Masetti, N., et al. 1999, GRB Coordinates Network, 327, 1

Masetti, N., et al. 2000a, A\&A, 354, 473

Masetti, N., Palazzi, E., Pian, E., Cosentino, R., Ghinassi, F., Magazzu, A., \& Benetti, S. 2000b, GRB Coordinates Network, 774, 1 
Masetti, N., Palazzi, E., Pedani, M., Magazzu, A., Ghinassi, F., \& Pian, E. 2001, GRB Coordinates Network, 926, 1

Masetti, N., et al. 2003, A\&A, 404, 465

Masetti, N., Palazzi, E., Rol, E., Pian, E., \& Pompei, E. 2004, GRB Coordinates Network, 2515,1

McBreen, S., Hanlon, L., McGlynn, S., McBreen, B., Foley, S., Preece, R., von Kienlin, A., \& Williams, O. R. 2006, A\&A, 455, 433

McBreen, S., et al. 2007a, GCNR, 46, 2 (2007), 46, 2

McBreen, S., et al. 2007b, GCNR, 101, 1 (2007), 101, 1

McGowan, K., et al. 2005, GRB Coordinates Network, 3317, 1

Melandri, A., et al. 2006a, A\&A, 451, 27

Melandri, A., et al. 2006b, GRB Coordinates Network, 5103, 1

Melandri, A., Gomboc, A., Smith, R. J., \& Tanvir, N. 2006c, GRB Coordinates Network, 5579,1

Melandri, A., Rol, E., \& Tanvir, N. 2007, GRB Coordinates Network, 6602, 1

Mereghetti, S., Götz, D., Borkowski, J., Walter, R., \& Pedersen, H. 2003a, A\&A, 411, L291

Mereghetti, S., et al. 2003b, ApJ, 590, L73

Mereghetti, S., et al. 2005a, A\&A, 433, 113

Mereghetti, S., Götz, D., Mowlavi, N., Shaw, S., Beck, M., Turler, M., \& Borkowski, J. 2005b, GRB Coordinates Network, 3348, 1

Mereghetti, S., Götz, D., Mowlavi, N., Eckert, D., Beck, M., \& Borkowski, J. 2005c, GRB Coordinates Network, 4327, 1

Mereghetti, S., \& Götz, D. 2006, GRB Coordinates Network, 4659, 1

Mereghetti, S., \& Gotz, D. 2006, GRB Coordinates Network, 5493, 1

Mereghetti, S., Götz, D., Shaw, S., Haymoz, P., Beck, M., \& Borkowski, J. 2006a, GRB Coordinates Network, 4505, 1 
Mereghetti, S., Paizis, A., Götz, D., Shaw, S., Beck, M., Kreykenbohm, I., \& Borkowski, J. 2006b, GRB Coordinates Network, 5751, 1

Mereghetti, S., Paizis, A., Götz, D., Petry, D., Mowlavi, N., Beck, M., \& Borkowski, J. 2006c, GRB Coordinates Network, 5834, 1

Mereghetti, S., Paizis, A., Götz, D., Kreykenbohm, I., Mowlavi, N., Beck, M., \& Borkowski, J. 2007a, GRB Coordinates Network, 6189, 1

Mereghetti, S., Paizis, A., Götz, D., Petry, D., Beckmann, V., Beck, M., \& Borkowski, J. 2007b, GRB Coordinates Network, 6823, 1

Metzger, M. R., Djorgovski, S. G., Kulkarni, S. R., Steidel, C. C., Adelberger, K. L., Frail, D. A., Costa, E., \& Frontera, F. 1997, Nature, 387, 878

Michałowski, M. J., Hjorth, J., Castro Cerón, J. M., \& Watson, D. 2008, ApJ, 672, 817

Milne, P. A. 2006, GRB Coordinates Network, 5127, 1

Milne, P. A. 2007, GRB Coordinates Network, 7056, 1

Mineo, T., Tagliaferri, G., Malesani, D., Giommi, P., Burrows, D., Fox, D., Chincarini, G., \& Page, K. 2005, GRB Coordinates Network, 4195, 1

Minezaki, T., Price, P. A., Yoshii, Y., Cowie, L., \& Kakazu, Y. 2007, GRB Coordinates Network, 7018, 1

Mirabal, N., Halpern, J. P., Gotthelf, E. V., \& Mukherjee, R. 2005, ApJ, 620, 379

Mirabal, N., Halpern, J. P., An, D., Thorstensen, J. R., \& Terndrup, D. M. 2006, ApJ, 643, L99

Mirabal, N., Halpern, J. P., \& O'Brien, P. T. 2007a, ApJ, 661, L127

Mirabal, N., Melandri, A., \& Halpern, J. P. 2007b, GRB Coordinates Network, 6162, 1

Misra, K., Kamble, A. P., Sahu, D. K., Srividya, S., Bama, P., Anupama, G. C., \& Vanniarajan, M. S. 2005, GRB Coordinates Network, 4259, 1

Misra, K., Bhattacharya, D., Sahu, D. K., Sagar, R., Anupama, G. C., Castro-Tirado, A. J., Guziy, S. S., \& Bhatt, B. C. 2007, A\&A, 464, 903

Mohan, V., et al. 2001, GRB Coordinates Network, 1120, 1

Monard, B., \& Price, A. 2005, GRB Coordinates Network, 3292, 1 
Monfardini, A., et al. 2005a, GRB Coordinates Network, 3001, 1

Monfardini, A., et al. 2005b, GRB Coordinates Network, 3503, 1

Monfardini, A., et al. 2005c, GRB Coordinates Network, 3588, 1

Monnelly, G., Dullighan, A., Butler, N., Vanderspek, R., \& Ricker, G. 2002, GRB Coordinates Network, 1339, 1

Moran, L., et al. 2005, A\&A, 432, 467

Moretti, A., Guidorzi, C., Romano, P., Barthelmy, S. D., Burrows, D. N., Roming, P., \& Gehrels, N. 2007, GCNR, 91, 1 (2007), 91, 1

Morgan, A. N., Chester, M. M., \& Pagani, C. 2006, GRB Coordinates Network, 5364, 1

Mundell, C. G., et al. 2007, ApJ, 660, 489

Murakami, T., Ueda, Y., Yoshida, A., Kawai, N., Marshall, F. E., Corbet, R. H. D., \& Takeshima, T. 1997, IAU Circ., 6732, 1

Nakagawa, Y. E., et al. 2006, PASJ, 58, L35

Nielsen, J., Jensen, B. L., Fynbo, J. P. U., Hjorth, J., \& Karoff, C. 2006, GRB Coordinates Network, 4620, 1

Nysewander, M., Moran, J., Johnson, L., Moschler, D., Reichart, D., \& Henden, A. 2002a, GRB Coordinates Network, 1760, 1

Nysewander, M., Reichart, D., \& Schwartz, M. 2002b, GRB Coordinates Network, 1699, 1

Nysewander, M., Henden, A., Lopez-Morales, M., Reichart, D., \& Schwartz, M. 2003, GRB Coordinates Network, 1861, 1

Nysewander, M., Bayliss, M., Foster, A., \& Reichart, D. 2004, GRB Coordinates Network, 2900,1

Nysewander, M., et al. 2005, GRB Coordinates Network, 3067, 1

Nysewander, M. C., et al. 2006, ApJ, 651, 994

Nysewander, M., Reichart, D. E., Crain, J. A., Foster, A., Haislip, J., Ivarsen, K., Lacluyze, A., \& Trotter, A. 2007, ArXiv e-prints, 708, arXiv:0708.3444

Oates, S. R., et al. 2006, MNRAS, 372, 327 
Oates, S. R., et al. 2007, MNRAS, 380, 270

Ofek, E. O., et al. 2007, ApJ, 662, 1129

Oksanen, A., et al. 2001, GRB Coordinates Network, 1019, 1

Olive, J.-F., et al. 2005, GRB Coordinates Network, 4124, 1

Osip, D., Chen, H.-W., \& Prochaska, J. X. 2006, GRB Coordinates Network, 5715, 1

Paciesas, W. S., et al. 1999, ApJS, 122, 465

Pagani, C., La Parola, V., \& Burrows, D. N. 2005, GRB Coordinates Network, 3934, 1

Pagani, C., Morris, D., \& Burrows, D. N. 2006, GRB Coordinates Network, 5393, 1

Pagani, C., Vetere, L., Racusin, J. L., Landsman, W., Barbier, L., \& Stamatikos, M. 2007a, GCNR, 53, 1 (2007), 53, 1

Pagani, C., Racusin, J. L., Kuin, N. P. M., Holland, S. T., Barthelmy, S. D., \& Gehrels, N. 2007b, GCNR, 97, 1 (2007), 97, 1

Page, M. J., Ziaeepour, H., Blustin, A. J., Chester, M., Fink, R., \& Gehrels, N. 2005, GRB Coordinates Network, 3859, 1

Page, K. L., Burrows, D. N., Rol, E., \& Boyd, P. 2006a, GRB Coordinates Network, 4796, 1

Page, K. L., Cummings, J., Hurley, K., Burrows, D. N., Morris, D. C., Kennea, J. A., Brown, P., \& Palmer, D. M. 2006b, GRB Coordinates Network, 5686, 1

Paizis, A., Mereghetti, S., Götz, D., Kreykenbohm, I., Mowlavi, N., Beck, M., \& Borkowski, J. 2007, GRB Coordinates Network, 6182, 1

Palazzi, E., et al. 1999a, GRB Coordinates Network, 262, 1

Palazzi, E., et al. 1999b, GRB Coordinates Network, 449, 1

Palazzi, E., et al. 2000a, GRB Coordinates Network, 691, 1

Palazzi, E., et al. 2000b, GRB Coordinates Network, 767, 1

Palmer, D., et al. 2006, GRB Coordinates Network, 4584, 1

Palmer, D., et al. 2007a, GRB Coordinates Network, 6643, 1

Palmer, D., et al. 2007b, GRB Coordinates Network, 6911, 1 
Pandey, S. B., et al. 2006a, A\&A, 460, 415

Pandey, S. B., de Pasquale, M., Page, M. J., \& Ziaeepour, H. Z. 2006b, GRB Coordinates Network, 5397, 1

Park, H. S., Porrata, R., Williams, G. G., Hartmann, D., Hurley, K., \& Barthelmy, S. 2000, GRB Coordinates Network, 628, 1

Parsons, A., et al. 2006, GRB Coordinates Network, 5761, 1

Pavlenko, E., \& Rumyantsev, V. 2002, GRB Coordinates Network, 1277, 1

Pedersen, H., Lindgren, B., Hjorth, J., Andersen, M. I., Jaunsen, A. O., Sollerman, J., Smoker, J., \& Mooney, C. 1999a, GRB Coordinates Network, 192, 1

Pedersen, H., Hjorth, J., Jensen, B. L., Rasmussen, M. B., Jaunsen, A. O., \& Hurley, K. 1999b, GRB Coordinates Network, 349, 1

Pedersen, H., Jensen, B. L., Gorosabel, J., \& Fynbo, J. P. U. 2000, GRB Coordinates Network, 617, 1

Pedersen, K., et al. 2006, ApJ, 636, 381

Pellizza, L. J., et al. 2006, A\&A, 459, L5

Penprase, B. E., et al. 2006, ApJ, 646, 358

Perley, D. A., Foley, R. J., Bloom, J. S., \& Butler, N. R. 2006, GRB Coordinates Network, 5387, 1

Perley, D. A., Thoene, C. C., \& Bloom, J. S. 2007a, GRB Coordinates Network, 6774, 1

Perley, D. A., Chornock, R., Bloom, J. S., Fassnacht, C., \& Auger, M. W. 2007b, GRB Coordinates Network, 6850, 1

Perley, D. A., Bloom, J. S., Modjaz, M., Poznanski, D., \& Thoene, C. C. 2007c, GRB Coordinates Network, 7140, 1

Perley, D. A., et al. 2008, ApJ, 672, 449

Perri, M., Tagliaferri, G., \& Burrows, D. N. 2006, GRB Coordinates Network, 4508, 1

Perri, M., Stratta, G., Fenimore, E., Schady, P., Barthelmy, S. D., Burrows, D. N., Roming, P., \& Gehrels, N. 2007a, GCNR, 103, 1 (2007), 103, 1 
Perri, M., Stratta, G., Kuin, N. P. M., Barthelmy, S. D., Burrows, D. N., Roming, P., \& Gehrels, N. 2007b, GCNR, 102, 2 (2007), 102, 2

Piccioni, A., Bartolini, C., Bruni, I., de Blasi, A., Guarnieri, A., Giovannelli, F., \& Pizzichini, G. 2002, GRB Coordinates Network, 1486, 1

Piranomonte, S., Covino, S., Malesani, D., Tagliaferri, G., Chincarini, G., \& Stella, L. 2006a, GRB Coordinates Network, 5392, 1

Piranomonte, S., et al. 2006b, GRB Coordinates Network, 4520, 1

Piranomonte, S., Vergani, S., D’Avanzo, P., \& Tagliaferri, G. 2007, GRB Coordinates Network, 6612,1

Piro, L., et al. 2002, ApJ, 577, 680

Pozanenko, A., Shulga, A., Volnova, A., Msu, S., Ibrahimov, M., \& Karimov, R. 2006, GRB Coordinates Network, 5902, 1

Pozanenko, A., Shulga, A., Volnova, A., Hafizov, B., Asfandiyarov, I., \& Ibrahimov, M. 2007, GRB Coordinates Network, 6407, 1

Price, P., Axelrod, T., \& Schmidt, B. 2000a, GRB Coordinates Network, 640, 1

Price, P., Axelrod, T., \& Schmidt, B. 2000b, GRB Coordinates Network, 780, 1

Price, P. A., Meltzer, J., Stalder, B., Djorgovski, S. G., \& Galama, T. J. 2000c, GRB Coordinates Network, 893, 1

Price, P. A., Axelrod, T. S., \& Schmidt, B. P. 2000d, GRB Coordinates Network, 898, 1

Price, P. A., Morrison, G., \& Bloom, J. S. 2001a, GRB Coordinates Network, 919, 1

Price, P. A., Axelrod, T. S., Schmidt, B. P., \& Reichart, D. E. 2001b, GRB Coordinates Network, 1020, 1

Price, P. A., Axelrod, T. S., Schmidt, B. P., Reichart, D. E., Bloom, J. S., \& Fox, D. 2001c, GRB Coordinates Network, 1017, 1

Price, P. A., Gorham, P., Yost, S. A., \& Kulkarni, S. R. 2001d, GRB Coordinates Network, 1039, 1

Price, P. A., Jones, B., \& Peterson, B. A. 2002a, GRB Coordinates Network, 1289, 1

Price, P. A., Schmidt, B. P., \& Axelrod, T. S. 2002b, GRB Coordinates Network, 1379, 1 
Price, P. A., Schmidt, B. P., \& Axelrod, T. S. 2002c, GRB Coordinates Network, 1410, 1

Price, P. A., Schmidt, B. P., \& Axelrod, T. S. 2002d, GRB Coordinates Network, 1533, 1

Price, P. A., et al. 2002e, ApJ, 571, L121

Price, P. A., et al. 2002f, ApJ, 573, 85

Price, P. A., Bloom, J. S., Goodrich, R. W., Barth, A. J., Cohen, M. H., \& Fox, D. W. 2002g, GRB Coordinates Network, 1475, 1

Price, P. A., Hauser, M., Puhlhofer, G., \& Wagner, S. J. 2004, GRB Coordinates Network, 2639, 1

Price, P. A. 2006, GRB Coordinates Network, 5104, 1

Price, P. A., Berger, E., Fox, D. B., Cenko, S. B., \& Rau, A. 2006, GRB Coordinates Network, 5077, 1

Prieto, J., et al. 2007, GRB Coordinates Network, 6374, 1

Prigozhin, G., et al. 2005, GRB Coordinates Network, 3402, 1

Prochaska, J. X., Bloom, J. S., Chen, H. W., Hurley, K., Dressler, A., \& Osip, D. 2003, GRB Coordinates Network, 2482, 1

Prochaska, J. X., Ellison, S., Foley, R. J., Bloom, J. S., \& Chen, H.-W. 2005a, GRB Coordinates Network, 3332, 1

Prochaska, J. X., Bloom, J. S., Wright, J. T., Butler, R. P., Chen, H. W., Vogt, S. S., \& Marcy, G. W. 2005b, GRB Coordinates Network, 3833, 1

Prochaska, J. X., Foley, R., Tran, H., Bloom, J. S., \& Chen, H.-W. 2006, GRB Coordinates Network, 4593, 1

Prochaska, J. X., Thoene, C. C., Malesani, D., Fynbo, J. P. U., \& Vreeswijk, P. M. 2007a, GRB Coordinates Network, 6698, 1

Prochaska, J. X., Perley, D. A., Modjaz, M., Bloom, J. S., Poznanski, D., \& Chen, H.-W. 2007b, GRB Coordinates Network, 6864, 1

Pugliese, G., et al. 2005, A\&A, 439, 527

Quimby, R., Fox, D., Hoeflich, P., Roman, B., \& Wheeler, J. C. 2005, GRB Coordinates Network, 4221, 1 
Racusin, J. L., \& Barthelmy, S. D. 2007, GCNR, 84, 1 (2007), 84, 1

Racusin, J., Cummings, J., \& Schady, P. 2007, GCNR, 98, 2 (2007), 98, 2

Rau, A., et al. 2004, A\&A, 427, 815

Rau, A., Salvato, M., \& Greiner, J. 2005, A\&A, 444, 425

Reichart, D. E., et al. 1999, ApJ, 517, 692

Resmi, L., et al. 2005, A\&A, 440, 477

Retter, A., Kennea, J., Burrows, D., Grupe, D., Nousek, J., \& Gehrels, N. 2006, GRB Coordinates Network, 4434, 1

Rhoads, J., Wilson, A., Storchi-Bergmann, T., \& Fruchter, A. 2000, GRB Coordinates Network, 564, 1

Rhoads, J. E., \& Fruchter, A. S. 2001, ApJ, 546, 117

Rol, E., Salamanca, I., Kaper, L., Vreeswijk, P., Lacerda, P., Hodgkin, S., Tzanavaris, P., \& Tanvir, N. 2001, GRB Coordinates Network, 955, 1

Rol, E., \& Page, K. L. 2006, GRB Coordinates Network, 5406, 1

Rol, E., Tanvir, N., \& Mundell, C. 2006a, GRB Coordinates Network, 4855, 1

Rol, E., Jakobsson, P., Tanvir, N., \& Levan, A. 2006b, GRB Coordinates Network, 5555, 1

Rol, E., et al. 2007a, MNRAS, 374, 1078

Rol, E., et al. 2007b, ApJ, 669, 1098

Rol, E., et al. 2007c, GRB Coordinates Network, 6221, 1

Rol, E., Levan, A., Tanvir, N., Schirmer, M., \& Castro-Tirado, A. J. 2007d, GRB Coordinates Network, 6174, 1

Roy, R., Misra, K., \& Pandey, S. B. 2007e, GRB Coordinates Network, 6880, 1

Rumyantsev, V., Biryukov, V., Pozanenko, A., \& Ibrahimov, M. 2005a, GRB Coordinates Network, 4094, 1

Rumyantsev, V., Biryukov, V., Pozanenko, A., \& Ibrahimov, M. 2005b, GRB Coordinates Network, 4087, 1 
Rumyantsev, V., \& Pozanenko, A. 2007, GRB Coordinates Network, 6874, 1

Rykoff, E. 2004a, GRB Coordinates Network, 2915, 1

Rykoff, E. 2004b, GRB Coordinates Network, 2919, 1

Rykoff, E. S., et al. 2004a, ApJ, 601, 1013

Rykoff, E., McKay, T., \& Swan, H. 2004b, GRB Coordinates Network, 2912, 1

Rykoff, E. S., Rujopakarn, W., Yost, S. A., \& Yuan, F. 2005, GRB Coordinates Network, 3779,1

Rykoff, E. S., et al. 2006, ApJ, 638, L5

Rykoff, E. S., Yuan, F., \& Yost, S. A. 2007, GRB Coordinates Network, 6497, 1

Sahu, K. C., et al. 2000, ApJ, 540, 74

Sakamoto, T., et al. 2005a, GRB Coordinates Network, 3189, 1

Sakamoto, T., et al. 2005b, ApJ, 629, 311

Sakamoto, T., Marshall, F., Stratta, G., Perri, M., Barthelmy, S. D., Burrows, D., Roming, P., \& Gehrels, N. 2006, GCNR, 19, 1 (2006), 19, 1

Sakamoto, T., Parsons, A., Page, K., Barthelmy, S. D., Burrows, D. N., Roming, P., \& Gehrels, N. 2007, GCNR, 95, 2 (2007), 95, 2

Sakamoto, T., et al. 2008, ApJS, 175, 179

Salamanca, I., Rol, E., Wijers, R., Ellison, S., Kaper, L., \& Tanvir, N. 2002, GRB Coordinates Network, 1611, 1

Saracco, P., et al. 2001, GRB Coordinates Network, 1205, 1

Sato, G., et al. 2007, GRB Coordinates Network, 7148, 1

Sbarufatti, B., et al. 2007, GCNR, 67, 1 (2007), 67, 1

Schady, P. 2006, GRB Coordinates Network, 5413, 1

Schady, P., \& Moretti, A. 2006, GRB Coordinates Network, 5296, 1

Schady, P., \& Parsons, A. M. 2007, GRB Coordinates Network, 6712, 1 
Schady, P., Evans, P. A., \& Starling, R. L. C. 2007, GCNR, 87, 1 (2007), 87, 1

Schaefer, B. E., et al. 1999, ApJ, 524, L103

Schaefer, J., Savage, S., Hwang, S., Canterna, R., Nysewander, M., \& Reichart, D. 2002a, GRB Coordinates Network, 1681, 1

Schaefer, J., Savage, S., Canterna, R., Nysewander, M., Reichart, D., Henden, A., \& Lamb, D. 2002b, GRB Coordinates Network, 1776, 1

Schaefer, J., Gibbs, D., Nysewander, M., Canterna, R., \& Reichart, D. 2003, GRB Coordinates Network, 2137, 1

Shakhovskoy, D., Rumyantsev, V., Biryukov, V., \& Pozanenko, A. 2007, GRB Coordinates Network, 6485, 1

Sharapov, D., Abdullaeva, G., Ibrahimov, M., \& Pozanenko, A. 2005a, GRB Coordinates Network, 4049, 1

Sharapov, D., Ibrahimov, M., Pozanenko, A., \& Rumyantsev, V. 2005b, GRB Coordinates Network, 4185, 1

Sharapov, D., Ibrahimov, M., Pozanenko, A., \& Rumyantsev, V. 2006a, GRB Coordinates Network, 4927, 1

Sharapov, D., Ibrahimov, M., \& Pozanenko, A. 2006b, GRB Coordinates Network, 4925, 1

Sharapov, D., Pozanenko, A., Klunko, E., Ibrahimov, M., \& Rumyantsev, V. 2006c, GRB Coordinates Network, 4956, 1

Sharapov, D., Augusteijn, T., \& Pozanenko, A. 2006d, GRB Coordinates Network, 5263, 1

Shin, M.-S., \& Berger, E. 2007, ApJ, 660, 1146

Silvey, J., Allen, D., Canterna, R., \& Price, P. A. 2003, GRB Coordinates Network, 2447, 1

Smith, D. A., Rykoff, E. S., \& Yost, S. A. 2005, GRB Coordinates Network, 3021, 1

Soderberg, A., Djorgovski, S. G., Halpern, J. P., \& Mirabal, N. 2004a, GRB Coordinates Network, 2837, 1

Soderberg, A. M., Berger, E., \& Ofek, E. 2005, GRB Coordinates Network, 4186, 1

Soderberg, A. M., et al. 2006a, ApJ, 650, 261 
Soderberg, A. M., et al. 2007, ApJ, 661, 982

Sollerman, J., et al. 2007, A\&A, 466, 839

Soyano, T., Mito, H., \& Urata, Y. 2006, GRB Coordinates Network, 5548, 1

Stamatikos, M., et al. 2006, GRB Coordinates Network, 5760, 1

Stamatikos, M., et al. 2007a, GRB Coordinates Network, 6711, 1

Stamatikos, M., Evans, P. A., Brown, P. J., Barthelmy, S. D., Burrows, D. N., Roming, P., \& Gehrels, N. 2007b, GCNR, 107, 2 (2007), 107, 2

Stanek, K. Z., Garnavich, P. M., Jha, S., \& Berlind, P. 2000, GRB Coordinates Network, 709,1

Stanek, K. Z., et al. 2005, ApJ, 626, L5

Stanek, K. Z., et al. 2007, ApJ, 654, L21

Starling, R., Osborne, J. P., Page, K. L., \& Marshall, F. E. 2007, GRB Coordinates Network, 6852,1

Starling, R. L. C., et al. 2007b, GCNR, 66, 3 (2007), 66, 3

Stefanescu, A., Schrey, F., Kanbach, G., Duscha, S., Muhlegger, M., Prymak, N., \& Steinle, H. 2006a, GRB Coordinates Network, 5291, 1

Stefanescu, A., Schrey, F., Duscha, S., Kanbach, G., Muehlegger, M., Primak, N., \& Steinle, H. 2006b, GRB Coordinates Network, 5623, 1

Stefanescu, A., Duscha, S., Kanbach, G., Schrey, F., Slowikowska, A., \& Steinle, H. 2007, GRB Coordinates Network, 6723, 1

Stern, D., Perley, D. A., Reddy, N., Prochaska, J. X., Spinrad, H., \& Dickinson, M. 2007, GRB Coordinates Network, 6928, 1

Still, M., et al. 2005, ApJ, 635, 1187

Stratta, G., et al. 2007a, A\&A, 461, 485

Stratta, G., et al. 2007b, A\&A, 474, 827

Stratta, G., Perri, M., Krimm, H., Oates, S. R., Barthelmy, S. D., Burrows, D. N., Roming, P., \& Gehrels, N. 2007c, GCNR, 104, 2 (2007), 104, 2 
Stratta, G., Perri, M., Conciatore, M., Burrows, D., \& Sato, G. 2007d, GRB Coordinates Network, 6119, 1

Stroh, M. C., et al. 2007, GCNR, 99, 1 (2007), 99, 1

Swan, H., Smith, I., Akerlof, C., Yost, S., \& Skinner, M. 2006, GRB Coordinates Network, 4491, 1

Swan, H., Smith, I., Akerlof, C., \& Skinner, M. 2007, GRB Coordinates Network, 6472, 1

Tagliaferri, G., et al. 2005, GRB Coordinates Network, 4222, 1

Tam, P. H., Pun, C. S. J., Huang, Y. F., \& Cheng, K. S. 2005, New Astronomy, 10, 535

Tanvir, N., Rol, E., \& Hewett, P. 2006, GRB Coordinates Network, 5216, 1

Terada, H., Pyo, T.-S., Aoki, K., Hattori, T., \& Kawai, N. 2007, GRB Coordinates Network, 6976, 1

Terra, F., et al. 2006, GRB Coordinates Network, 5659, 1

Terra, F., et al. 2007, GRB Coordinates Network, 6458, 1

Thone, C., et al. 2005, GRB Coordinates Network, 4291, 1

Thoene, C. C., Nilsson, K., Jensen, B. L., \& Fynbo, J. 2006a, GRB Coordinates Network, 5003,1

Thoene, C. C., Jensen, B. L., Fynbo, J., \& Solheim, J.-E. 2006b, GRB Coordinates Network, 5094,1

Thoene, C. C., et al. 2006c, GRB Coordinates Network, 5373, 1

Thoene, C. C., Fynbo, J. P. U., Jakobsson, P., Vreeswijk, P. M., \& Hjorth, J. 2006d, GRB Coordinates Network, 5812, 1

Thoene, C. C., Malesani, D., Fynbo, J. P. U., Henriksen, C., \& Sharapov, D. 2006e, GRB Coordinates Network, 5807, 1

Thoene, C. C., Perley, D. A., \& Bloom, J. S. 2007a, GRB Coordinates Network, 6663, 1

Thoene, C. C., Kann, D. A., Augusteijn, T., \& Reyle-Laffont, C. 2007b, GRB Coordinates Network, 6154, 1 
Thoene, C. C., Jaunsen, A. O., Fynbo, J. P. U., Jakobsson, P., \& Vreeswijk, P. M. 2007c, GRB Coordinates Network, 6379, 1

Thoene, C. C., Jakobsson, P., Fynbo, J. P. U., Malesani, D., Hjorth, J., \& Vreeswijk, P. M. 2007d, GRB Coordinates Network, 6499, 1

Thoene, C. C., Perley, D. A., Cooke, J., Bloom, J. S., Chen, H.-W., \& Barton, E. 2007e, GRB Coordinates Network, 6741, 1

Thorstensen, J., Uglesich, R., Halpern, J., Mirabal, N., Costa, E., Feroci, M., \& Piro, L. 1999, GRB Coordinates Network, 423, 1

Tiengo, A., Mereghetti, S., Ghisellini, G., Rossi, E., Ghirlanda, G., \& Schartel, N. 2003, A\&A, 409, 983

Tinney, C., et al. 1998, IAU Circ., 6896, 1

Tristram, P., Jelinek, M., de Ugarte Postigo, A., Gorosabel, J., Castro-Tirado, A. J., Castro Cerón, J. M., \& Yock, P. 2005a, GRB Coordinates Network, 3151, 1

Tristram, P., Jelinek, M., Castro-Tirado, A. J., de Ugarte Postigo, A., Guziy, S., Gorosabel, J., Yock, P., \& Krimm, H. 2005b, GRB Coordinates Network, 4055, 1

Uglesich, R., Mirabal, N., Halpern, J. P., Vanlandingham, K., Noel-Storr, J., Wagner, R. M., \& Eskridge, P. 2000, GRB Coordinates Network, 663, 1

Ukwatta, T. N., et al. 2007, GCNR, 106, 2 (2007), 106, 2

Updike, A. C., Garimella, K. V., \& Hartmann, D. H. 2006, GRB Coordinates Network, 5738, 1

Updike, A. C., Puls, J., Hartmann, D. H., Wood, M., Cardenzana, J., \& Pederson, S. 2007, GRB Coordinates Network, 6530, 1

Urata, Y., et al. 2004, GRB Coordinates Network, 2662, 1

Valle, M. D., et al. 2006, Nature, 444, 1050

Vergani, S. D., et al. 2007, GCNR, 39, 2 (2007), 39, 2

Vetere, L., Cummings, J., \& Immler, S. 2007a, GCNR, 29, 1 (2007), 29, 1

Vetere, L., Cummings, J., Holland, S. T., Bartthelmy, S. D., Burrows, D. N., Roming, P., \& Gehrels, N. 2007b, GCNR, 55, 1 (2007), 55, 1 
Villasenor, J. S., et al. 2005, Nature, 437, 855

Vrba, F. J., et al. 2000, ApJ, 528, 254

Vreeswijk, P. M., et al. 1999a, ApJ, 523, 171

Vreeswijk, P. M., et al. 1999b, GRB Coordinates Network, 324, 1

Vreeswijk, P. M., et al. 1999c, GRB Coordinates Network, 496, 1

Vreeswijk, P., Rol, E., Packham, C., Tanvir, N., Kouveliotou, C., Wijers, R., \& Knapen, J. 2000, GRB Coordinates Network, 886, 1

Vreeswijk, P. M., et al. 2001, ApJ, 546, 672

Vreeswijk, P., Fruchter, A., Hjorth, J., \& Kouveliotou, C. 2003, GRB Coordinates Network, 1785,1

Vreeswijk, P. M., et al. 2004, A\&A, 419, 927

Vreeswijk, P., \& Jaunsen, A. 2006, GRB Coordinates Network, 4974, 1

Vreeswijk, P. M., et al. 2006, A\&A, 447, 145

Wiersema, K., Starling, R. L. C., Rol, E., Vreeswijk, P., \& Wijers, R. A. M. J. 2004, GRB Coordinates Network, 2800, 1

Wiersema, K., Thoene, C. C., \& Rol, E. 2006, GRB Coordinates Network, 5552, 1

Wiersema, K., Rol, E., \& Thoene, C. C. 2007, GRB Coordinates Network, 6188, 1

Wiersema, K., et al. 2008, in preparation

Xin, L. P., Zhai, M., Qiu, Y. L., Wei, J. Y., Hu, J. Y., Deng, J. S., Urata, Y., \& Zheng, W. K. 2007a, GRB Coordinates Network, 6747, 1

Xin, L. P., et al. 2007b, GRB Coordinates Network, 6965, 1

Xin, L. P., et al. 2007c, GRB Coordinates Network, 6956, 1

Xin, L. P., Zhai, M., Qiu, Y. L., Wei, J. Y., Hu, J. Y., Deng, J. S., Wang, J., \& Zheng, W. K. 2007d, GRB Coordinates Network, 7015, 1

Yanagisawa, K., Toda, H., \& Kawai, N. 2006a, GRB Coordinates Network, 4496, 1

Yanagisawa, K., Toda, H., \& Kawai, N. 2006b, GRB Coordinates Network, 4517, 1 
Yoshida, A., et al. 2002, GRB Coordinates Network, 1686, 1

Yoshida, M., Yanagisawa, K., \& Kawai, N. 2007a, GRB Coordinates Network, 6123, 1

Yoshida, M., Yanagisawa, K., Shimizu, Y., Nagayama, S., Toda, H., \& Kawai, N. 2007b, GRB Coordinates Network, 7119, 1

Yost, S. A., et al. 2006, ApJ, 636, 959

Zheng, W. K., et al. 2007, GRB Coordinates Network, 6250, 1

Ziaeepour, H., Barthelmy, S. D., Parsons, A., Page, K. L., de Pasquale, M., \& Schady, P. 2007a, GCNR, 74, 2 (2007), 74, 2

Ziaeepour, H., Barthelmy, S. D., Sakamoto, T., Evans, P. A., Page, K. L., \& Schady, P. 2007b, GCNR, 72, 2 (2007), 72, 2

Zimmerman, N., \& Tyagi, S. 2006, GRB Coordinates Network, 4440, 1

Zhu, J. 2000, GRB Coordinates Network, 904, 1

Zhu, J. 2001, GRB Coordinates Network, 946, 1 